\title{
The Dense Molecular Ridge in NGC 2024 \\ by
}

Tomomi Watanabe

A Thesis Submitted to Saint Mary's University, Halifax, Nova Scotia in Partial Fulfillment of the Requirements for the Degree of

MASTER OF SCIENCE

in

Astronomy

(Department of Astronomy and Physics)

August 20, 2007, Halifax, Nova Scotia

(c) Tomomi Watanabe, 2007

supervisors name

George M. Mitchell

examiner 1's name

David A. Clarke

examiner 2's name

Marcin J. Sawicki

Date: $\quad$ August 20, 2007 


$\begin{array}{ll}\begin{array}{l}\text { Library and } \\ \text { Archives Canada }\end{array} & \begin{array}{l}\text { Bibliothèque et } \\ \text { Archives Canada }\end{array} \\ \begin{array}{l}\text { Published Heritage } \\ \text { Branch }\end{array} & \begin{array}{l}\text { Direction du } \\ \text { Patrimoine de l'édition }\end{array} \\ \begin{array}{l}\text { 395 Wellington Street } \\ \text { Ottawa ON K1A ON4 } \\ \text { Canada }\end{array} & \begin{array}{l}\text { 395, rue Wellington } \\ \text { Ottawa ON K1A ON4 } \\ \text { Canada }\end{array}\end{array}$

Your file Votre référence ISBN: 978-0-494-35780-4 Our file Notre référence ISBN: $978-0-494-35780-4$

NOTICE:

The author has granted a nonexclusive license allowing Library and Archives Canada to reproduce, publish, archive, preserve, conserve, communicate to the public by telecommunication or on the Internet, loan, distribute and sell theses worldwide, for commercial or noncommercial purposes, in microform, paper, electronic and/or any other formats.

The author retains copyright ownership and moral rights in this thesis. Neither the thesis nor substantial extracts from it may be printed or otherwise reproduced without the author's permission.
AVIS:

L'auteur a accordé une licence non exclusive permettant à la Bibliothèque et Archives Canada de reproduire, publier, archiver, sauvegarder, conserver, transmettre au public par télécommunication ou par l'Internet, prêter, distribuer et vendre des thèses partout dans le monde, à des fins commerciales ou autres, sur support microforme, papier, électronique et/ou autres formats.

L'auteur conserve la propriété du droit d'auteur et des droits moraux qui protège cette thèse. $\mathrm{Ni}$ la thèse ni des extraits substantiels de celle-ci ne doivent être imprimés ou autrement reproduits sans son autorisation.
In compliance with the Canadian

Privacy Act some supporting forms may have been removed from this thesis.

While these forms may be included in the document page count, their removal does not represent any loss of content from the thesis.
Conformément à la loi canadienne sur la protection de la vie privée, quelques formulaires secondaires ont été enlevés de cette thèse.

Bien que ces formulaires aient inclus dans la pagination, il n'y aura aucun contenu manquant. 


\section{Contents}

Contents $\ldots \ldots \ldots \ldots \ldots \ldots \ldots \ldots \ldots \ldots \ldots \ldots \ldots \ldots \ldots \ldots \ldots$

LIST of FIGURES $\ldots \ldots \ldots \ldots \ldots \ldots \ldots \ldots \ldots \ldots \ldots \ldots \ldots \ldots$

LIST of TABLES $\ldots \ldots \ldots \ldots \ldots \ldots \ldots \ldots \ldots \ldots \ldots \ldots \ldots \ldots \ldots \ldots \ldots$ vii

ACKNOWLEDGEMENTS $\ldots \ldots \ldots \ldots \ldots \ldots \ldots \ldots \ldots \ldots \ldots \ldots \ldots \ldots$

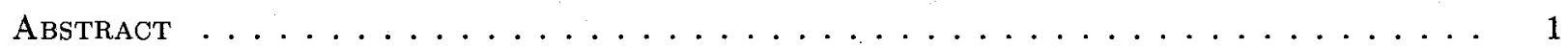

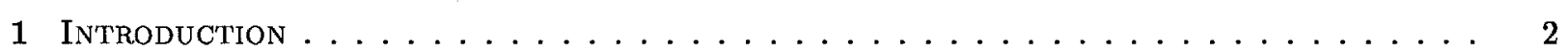

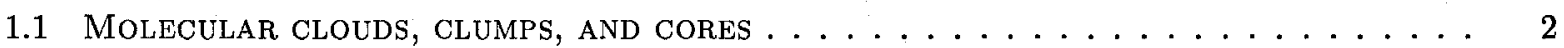

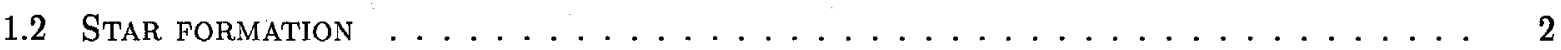

1.2.1 Young Stellar ObJects $\ldots \ldots \ldots \ldots \ldots \ldots \ldots \ldots \ldots$

1.3 ORION B AND NGC $2024 \ldots \ldots \ldots \ldots \ldots \ldots \ldots \ldots \ldots \ldots \ldots$

1.3 .1 Previous studies $\ldots \ldots \ldots \ldots \ldots \ldots \ldots \ldots \ldots \ldots \ldots$

1.4 Physical PRoPERTIES: TEMPERATURE ANd DENSITY $\ldots \ldots \ldots \ldots \ldots \ldots \ldots$

1.4.1 Temperature of FIR cores $\ldots \ldots \ldots \ldots \ldots \ldots \ldots \ldots \ldots$

1.4.2 FormaLdehyde AS TEMPERATURE PROBE $\ldots \ldots \ldots \ldots \ldots \ldots$

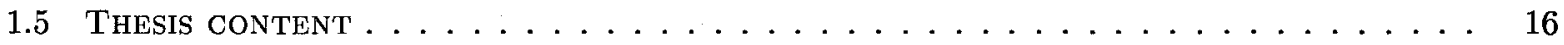

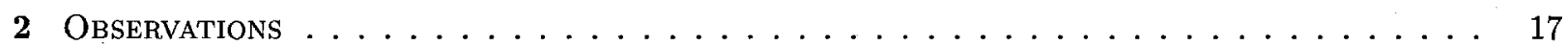

$2.1 \mathrm{H}_{2} \mathrm{CO} 3_{03} \rightarrow 2_{02}$ AND $3_{22} \rightarrow 2_{21}$ MAPS $\ldots \ldots \ldots \ldots \ldots \ldots \ldots \ldots \ldots \ldots \ldots \ldots \ldots$

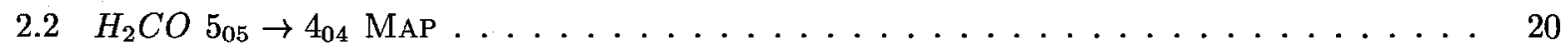

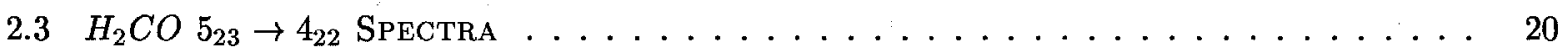

2.3.1 $\mathrm{HCO}^{+} \mathrm{J}=3-2$ AND $\mathrm{H}^{13} \mathrm{CO}^{+} \mathrm{J}=3-2$ SPECTRA $\ldots \ldots \ldots \ldots \ldots \ldots$

2.4 CUTS THROUGH FIR CORE POSITIONS $\ldots \ldots \ldots \ldots \ldots \ldots \ldots \ldots \ldots \ldots$ 


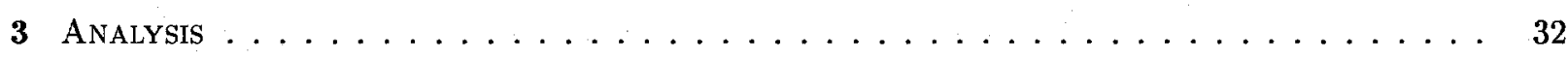

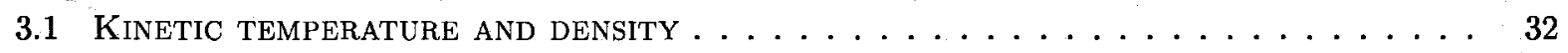

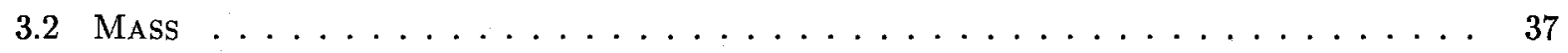

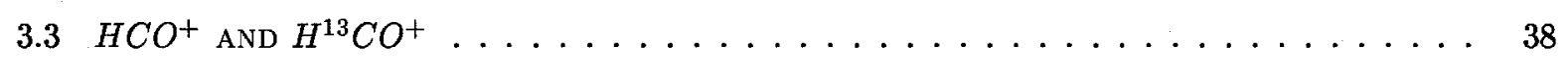

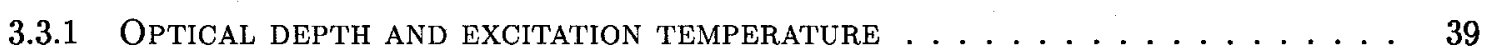

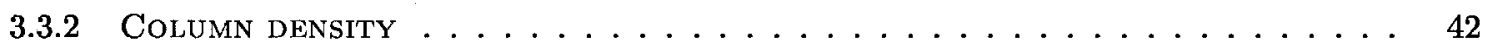

3.4 Column density fRom EXTINCTION ANd $850 \mu \mathrm{M} \ldots \ldots \ldots \ldots \ldots \ldots$

3.5 Fractional AbUndance $\ldots \ldots \ldots \ldots \ldots \ldots \ldots \ldots$

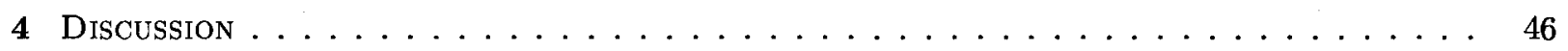

4.1 VARIATION OF PHYSICAL PROPERTIES THROUGHOUT RIDGE $\ldots \ldots \ldots \ldots \ldots$

4.1 .1 Temperature $\ldots \ldots \ldots \ldots \ldots \ldots \ldots \ldots \ldots \ldots$

4.1 .2 DenstTy $\ldots \ldots \ldots \ldots \ldots \ldots \ldots \ldots \ldots \ldots \ldots$

4.2 TeMPERATURE COMPARISON $\ldots \ldots \ldots \ldots \ldots \ldots \ldots \ldots \ldots$

4.3 STRUCTURE AND MORPHOLOGY $\ldots \ldots \ldots \ldots \ldots \ldots \ldots \ldots$

4.3.1 CoMparison WITH $H_{2} C O$ ABSORPTION $\ldots \ldots \ldots \ldots \ldots \ldots \ldots$

4.3.2 COMPARISON WITH DUST: FIR $1300 \mu \mathrm{M}$, SCUBA 850 AND $450 \mu \mathrm{M} \ldots \ldots \ldots$

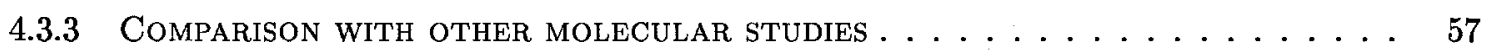

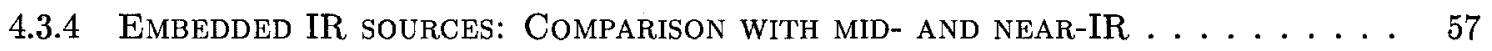

4.3.5 CoMPARISON WITH OPTICAL $\ldots \ldots \ldots \ldots \ldots \ldots \ldots$

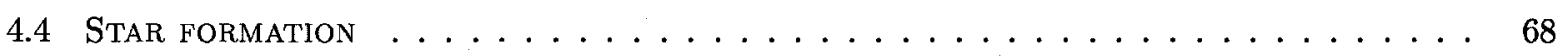

4.4 .1 VELOCITY INFORMATION $\ldots \ldots \ldots \ldots \ldots \ldots \ldots \ldots$

4.4 .2 STABILITY OF FIR CORES $\ldots \ldots \ldots \ldots \ldots \ldots \ldots \ldots \ldots$

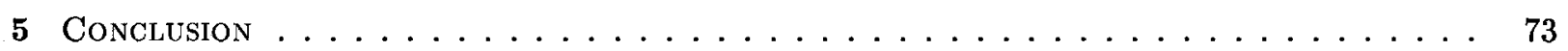

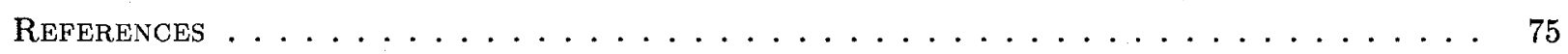




\section{List OF FIGURES}

1.1 Dense molecular gas in Orion B seen in $C S(2-1)$ from Lada et al. (1991a). The three northern-most components make up Orion B North, and the three southern-most components make up Orion B South. . . . . . . . . . . . . . . .

1.2 Optical image of NGC 2024 from the Digitized Sky Survey. The coordinates are displayed in epoch 2000. A dark lane of dust can be seen obscuring the center of the ionized region.

1.3 Dust continuum maps of Orion B south from SCUBA in $850 \mu \mathrm{m}$ (left) and $450 \mu \mathrm{m}$ (right), previously published in Johnstone et al. (2001), Mitchell et al. (2001), and Johnstone et al. (2006). The three major members of Orion B are labeled. The beam sizes are $15^{\prime \prime}$ for 850 $\mu \mathrm{m}$ and $15^{\prime \prime}$ for $450 \mu \mathrm{m}$.

2.1 Integrated intensity map of NGC 2024 ridge observed in $\mathrm{H}_{2} \mathrm{CO} 3_{03} \rightarrow 2_{02}$ line at 218.2222 $\mathrm{GHz}$. The intensity is integrated over the velocity range of $8-13 \mathrm{~km} \mathrm{~s}^{-1}$. The maximum value is at $12 \mathrm{~K} \mathrm{~km} \mathrm{~s}^{-1}$, and the minimum is at $0 \mathrm{~K} \mathrm{~km} \mathrm{~s}^{-1}$. The beam size is $23^{\prime \prime}$. The coordinates are in epoch 2000 , as are all the subsequent figures unless otherwise stated. . .

2.2 Integrated intensity map of NGC 2024 ridge observed in $\mathrm{H}_{2} \mathrm{CO}_{22} \rightarrow 2_{21}$ line at 218.4756 GHz. The intensity is integrated over the velocity range of $8-13 \mathrm{~km} \mathrm{~s}^{-1}$. The maximum value is at $5 \mathrm{~K} \mathrm{~km} \mathrm{~s}^{-1}$, and the minimum is at $0 \mathrm{~K} \mathrm{~km} \mathrm{~s}^{-1}$. The beam size is $23^{\prime \prime}$. . . .

2.3 Channel maps of $\mathrm{H}_{2} \mathrm{CO}_{303} \rightarrow 2_{02}$ transition. The velocity range is from 9 to $12.5 \mathrm{~km} \mathrm{~s}^{-1}$, taken at intervals of $0.5 \mathrm{~km} \mathrm{~s}^{-1} \ldots \ldots \ldots \ldots \ldots \ldots$

2.4 Integrated intensity map of NGC 2024 ridge observed in $\mathrm{H}_{2} \mathrm{CO} 5_{05} \rightarrow 4_{04}$ line at 362.7360 GHz. The intensity is integrated over the velocity range of $8-13 \mathrm{~km} \mathrm{~s}^{-1}$. The maximum value is at $10.4 \mathrm{~K} \mathrm{~km} \mathrm{~s}^{-1}$, and the minimum is at $0 \mathrm{~K} \mathrm{~km} \mathrm{~s}^{-1}$. The beam size is $15^{\prime \prime}$. . .

2.5 Contour channel maps of $\mathrm{H}_{2} \mathrm{CO} 5_{05} \rightarrow 4_{04}$ transition. The velocity range is from 9 to 12.5 $\mathrm{km} \mathrm{s}^{-1}$ at intervals of $0.5 \mathrm{~km} \mathrm{~s}^{-1}$. 
2.6 Contours of $\mathrm{H}_{2} \mathrm{CO} 5_{05} \rightarrow 4_{04}$ transition map plotted over $3_{03} \rightarrow 2_{02}$ transition map. The contours show intensities at $2,4,6,8$, and $10 \mathrm{~K} \mathrm{~km} \mathrm{~s}^{-1} \ldots \ldots \ldots \ldots$

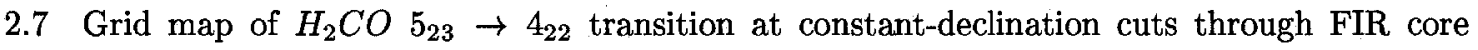
positions. The position of each spectrum in the grid corresponds to its true position within

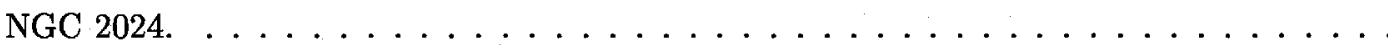

2.8 Spectra of $\mathrm{HCO}^{+} J=3-2$ line at $267.5576 \mathrm{GHz}$ (left) and the $\mathrm{H}^{13} \mathrm{CO}^{+} J=3-2$ line at $260.2555 \mathrm{GHz}$ at FIR core positions. The intensities are offset to display all the spectra simultaneously. The FIR positions are in order from FIR 1 (at top) to FIR 7 (at bottom). Note the difference in the $\mathrm{y}$-axis scales between the two plots. . . . . . . . . . . . 28

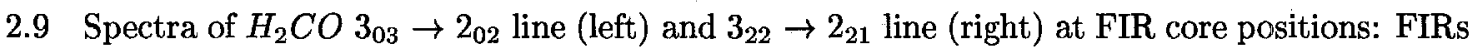
1-7 from top to bottom. Note the difference in the y-axis scales between the two plots. . .

2.10 Spectra of $\mathrm{H}_{2} \mathrm{CO} 5_{05} \rightarrow 4_{04}$ line (left) and the $5_{23} \rightarrow 4_{22}$ line (right) at FIR core positions: FIRs 1-7 from top to bottom. Note the difference in the y-axis scales between the two plots.

3.1 Best fitting Gaussian curves for spectra of $\mathrm{HCO}^{+}$lines at $267 \mathrm{GHz}$ to correct for selfabsorption at positions of FIR 1-7 (top to bottom).

4.1 Variation of temperature derived from $\mathrm{H}_{2} \mathrm{CO}$ line ratio $3_{03} \rightarrow 2_{02} / 3_{22} \rightarrow 2_{21}$ (left) and formaldehyde column density derived from $\mathrm{H}_{2} \mathrm{CO}_{302} \rightarrow 2_{02}$ line (right) across FIR cuts. The cuts are in order from FIR 1 to FIR 7 (top to bottom). Each cut is arbitrarily offset to display only its variations in comparison with the other cuts.

$4.2 \mathrm{H}_{2} \mathrm{CO} 3_{03} \rightarrow 2_{02}$ line map with labeled positions of VLA absorption measurements from Crutcher et al. (1986).

4.3 Cm absorption of $\mathrm{H}_{2} \mathrm{CO} 1_{10} \rightarrow 1_{11}$ line at $4.8297 \mathrm{GHz}$ from Crutcher et al. (1986) (left) and inverted emission spectra from $\mathrm{H}_{2} \mathrm{CO}_{303} \rightarrow 2_{02}$ line corresponding to all eight positions (right)

4.4 Contour map of $\mathrm{H}_{2} \mathrm{CO}_{303} \rightarrow 2_{02}$ transition. The positions of the FIR cores are indicated by red triangles. The contours represent intensities between 2 to $12 \mathrm{~K} \mathrm{~km} \mathrm{~s}^{-1}$, at intervals

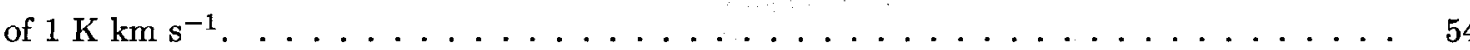


4.5 Contour map of $\mathrm{H}_{2} \mathrm{CO} 505_{5} \rightarrow 4_{04}$ transition. The positions of the FIR cores are indicated by red triangles. The contours represent intensities between 2 to $10 \mathrm{~K} \mathrm{~km} \mathrm{~s}^{-1}$, at intervals

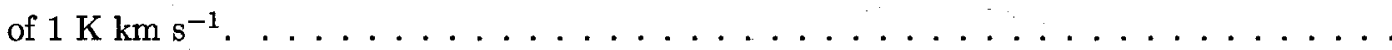

4.6 Contours of interpolated $\mathrm{H}_{2} \mathrm{CO}_{505} \rightarrow 4_{04}$ line plotted over gray scale map of $450 \mu \mathrm{m}$. The contours of the $5_{05} \rightarrow 4_{04}$ line display intensities between 1 and $10 \mathrm{~K} \mathrm{~km} \mathrm{~s}^{-1}$ at steps of $1 \mathrm{~K}$

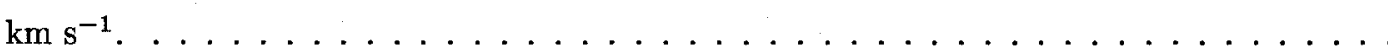

4.7 Contours of SCUBA $850 \mu \mathrm{m}$ map plotted over $\mathrm{H}_{2} \mathrm{CO} 3_{03} \rightarrow 22_{02}$ transition gray scale map. The $850 \mu \mathrm{m}$ contours represent intensities at $2,4,6,8,10$, and $12 \mathrm{~K} \mathrm{~km} \mathrm{~s}^{-1}$. The crosses

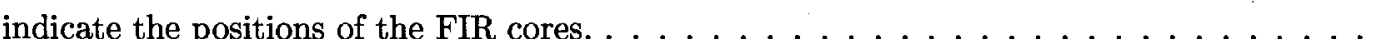

4.8 $\mathrm{H}_{2} \mathrm{CO}_{303} \rightarrow 2_{02}$ transition contours with positions of FIR cores, $C S$ cores from Schulz et al. (1991) and ionizing source IRS2b. The contours show intensities from 4 to $12 \mathrm{~K} \mathrm{~km} \mathrm{~s}^{-1}$ at

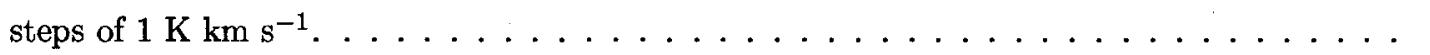

$4.9 \quad \mathrm{H}_{2} \mathrm{CO} 5_{05} \rightarrow 4_{04}$ transition contours with positions of FIR cores, $C S$ cores from Schulz et al. (1991) and the ionizing source IRS2b. The contours show intensities from 2, 4, 6, 7, 8, 9,

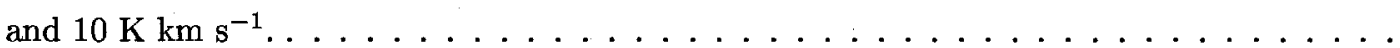

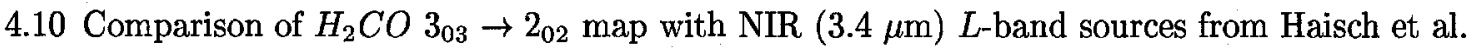
(2000). The plus signs indicate the positions of NIR sources, and the 7 open circles indicate the positions of the FIR cores. There are no exact coincidences of the NIR sources with the FIR cores. . . . . . . . . . . . . . . . . . . . .

4.11 Comparison of $\mathrm{H}_{2} \mathrm{CO}_{303} \rightarrow 2_{02}$ map with MIR $(10.8 \mu \mathrm{m}) \mathrm{N}$-band sources from Haisch et al. (2001). The MIR sources are labeled using the same source IDs as Haisch et al. (2001). . .

4.12 MSX $8 \mu \mathrm{m}$ with contours of $\mathrm{H}_{2} \mathrm{CO} 3_{03} \rightarrow 2_{02}$ line. The contours show intensities between 4 and $12 \mathrm{~K} \mathrm{~km} \mathrm{~s}^{-1}$ at steps of $2 \mathrm{~K} \mathrm{~km} \mathrm{~s}^{-1} .8 \mu \mathrm{m}$ traces the high temperature dust heated by ionization. The ionization front can be seen to coincide with the gap separating the northern clump and the southern clump. 
4.13 Fig.6 from Johnstone et al. (2006). NGC 2024 at (top-left) $450 \mu \mathrm{m}$, (top-right) optical from DSS, (bottom-left) $850 \mu \mathrm{m}$ with plus signs indicating the positions of the detected clumps and sizes indicating the calculated clump masses, and (bottom-right) $8 \mu \mathrm{m}$ showing the sharply

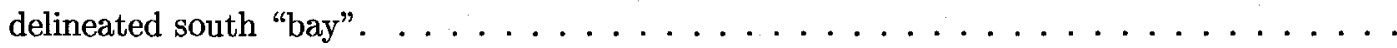

4.14 DSS image of NGC 2024 with contours of $\mathrm{H}_{2} \mathrm{CO}_{303} \rightarrow 2_{02}$ (top) and contours of SCUBA 850 $\mu \mathrm{m}$ (bottom). The $\mathrm{H}_{2} \mathrm{CO}$ contours show intensities at 2,4 , and $8 \mathrm{~K} \mathrm{~km} \mathrm{~s}^{-1}$. The SCUBA contours show intensities at $0.5,1,2,4$, and $10 \mathrm{~K} \mathrm{~km} \mathrm{~s}^{-1} . \ldots \ldots \ldots$ 


\section{LIST OF TABLES}

1.1 Summary of Temperature Results from Previous Studies . . . . . . . . . . . . . . . 14

2.1 Summary of Observed Formaldehyde Transitions $\ldots \ldots \ldots \ldots \ldots \ldots \ldots$

2.2 Positions of FIR Sources from Mezger et al. (1992) $\ldots \ldots \ldots \ldots \ldots \ldots$

2.3 Summary of Observed $\mathrm{HCO}^{+}$and $\mathrm{H}^{13} \mathrm{CO}^{+}$Lines $\ldots \ldots \ldots \ldots \ldots \ldots$

2.4 Integrated Intensities at FIR Core Positions for $\mathrm{H}_{2} \mathrm{CO}$ Transitions $\ldots \ldots \ldots \ldots \ldots$

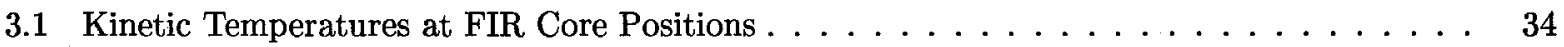

3.2 LTE and LVG Derived Temperature Comparison $\ldots \ldots \ldots \ldots \ldots \ldots$

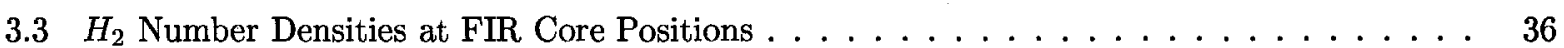

$3.4 \mathrm{H}_{2} \mathrm{CO}$ Column Densities at FIR Core Positions $\ldots \ldots \ldots \ldots \ldots \ldots \ldots \ldots$

3.5 Masses Derived for FIRs $3,4,5$, and $6 \ldots \ldots \ldots \ldots \ldots$

3.6 Optical Depths and Excitation Temperatures at FIR Core Positions . . . . . . . . . 41

$3.7 \mathrm{H}^{13} \mathrm{CO}^{+}$and $\mathrm{HCO}^{+}$Column Densities at FIR Core Positions $\ldots \ldots \ldots \ldots \ldots$

$3.8 H_{2}$ Column Densities from Extinction and $850 \mu \mathrm{m} \ldots \ldots \ldots \ldots \ldots \ldots \ldots$

3.9 Fractional Abundance for $\mathrm{H}_{2} \mathrm{CO}$ and $\mathrm{HCO}^{+}$at FIR Core Positions $\ldots \ldots \ldots$

4.1 Energy Terms of the Virial Stability Evaluation . . . . . . . . . . . . . . . 72 


\section{ACKNOWLEDGEMENTS}

For the duration of my thesis research, I frequently received help from a number of very generous people, and I would like to take this opportunity to show my appreciation for them. This thesis would not have been possible without the help from these individuals, and they made my experience at Saint Mary's University more valuable and rewarding.

I would like to thank my fellow graduate students, for having the patience to answer some of my elementary questions, especially about programming and Latex. In particular, Jon Ramsey who kindly plotted a figure in IDL for me, and Chris Geroux who I owe so much for help with Latex. I am also very grateful for my committee members, David Clarke and Marcin Sawicki, for agreeing to examine a Master's thesis outside their fields, and for the sudden arrangement for the defense.

Last, but not least, I would like to acknowledge George Mitchell, my thesis supervisor, for giving me the opportunity to work with him, even into retirement. I appreciate his generosity in guiding me throughout Hawaii, from the summit of Mauna Kea, to Volcanoes National Park, and even snorkeling. There are no words to justify my deep gratitude for these extraordinary experiences and memories I will cherish forever. 


\title{
Abstract
}

\section{The Dense Molecular Ridge in NGC 2024}

\author{
by Tomomi Watanabe
}

August 20, 2007

NGC 2024 is a star-forming region in Orion B which contains a dense molecular ridge and a number of dense cores seen by their dust emission (Mezger et al., 1992). This area is investigated using observations of four formaldehyde transitions, and the $J=3-2$ transition of $\mathrm{HCO}^{+}$and $\mathrm{H}^{13} \mathrm{CO}^{+}$. Maps of the ridge are produced and used to provide temperatures and densities, with a focus on the dense core positions. The resulting temperatures are very warm $(45-85 \mathrm{~K})$, and derived $\mathrm{H}_{2} \mathrm{CO}$ number densities are found to be $\sim 2 \times 10^{6} \mathrm{~cm}^{-3}$. Using the derived properties in combination with sub-mm data from Johnstone et al. (2006), core masses of $\sim 2 \mathrm{M}_{\odot}$ are found within a $15^{\prime \prime}$ beamsize, and $H_{2} C O$ fractional abundances are calculated to resemble previous values found in similar star-forming regions. $\mathrm{HCO}^{+}$and $\mathrm{H}^{13} \mathrm{CO}^{+}$observations are used to obtain temperatures and also optical depths. The virial theorem is applied to FIR cores $3,4,5$ and 6 suggests that all four cores are unstable against gravitational collapse. 


\section{InTRODUCTION}

\subsection{Molecular Clouds, Clumps, AND CORES}

Star formation occurs within gravitationally bound, large mixtures of gas and dust known as molecular clouds. In Giant Molecular Clouds (GMCs), such as the Orion B Complex, the mass typically ranges around $10^{4}-10^{6} M_{\odot}$. The kinetic temperatures found within GMC complexes are around $20 \mathrm{~K}$ with number densities $n\left(H_{2}\right) \sim 10^{2} \mathrm{~cm}^{-3}$. Molecules such as $H_{2}$ are formed when they freeze out of the gasphase and condense onto the dust grains that are intermixed within the clouds. The dust acts as a shield for the molecules and protects them against stellar radiation, allowing their formation. Due to such factors as photodissociation, ionization by nearby stars, turbulence, and shocks, the temperature and density can vary widely within GMCs. Substructures have been observed in forms of filaments, sheets, bubbles, and clumps. These complex structures make up the regions which are dense enough for star formation to take place. Clumps are defined as the denser regions within GMCs, such as NGC 2024 found within the Orion B complex. On a smaller scale, substructures are found within clumps which have even higher densities. These compact objects are often referred to as cores, which are most likely active or eventual sites of star formation. NGC 2024 is an example of a clump within Orion B where a string of cores resides. Compared to isolated cores which are more extensively studied due to their observational advantages (Section 4.4), these non-isolated cores are more difficult to investigate. These cores are optimal in studying the environments in which a group of stars forms from a single common clump. From careful evaluations of their physical properties using observations, each core may reveal its potential to form a star and the properties of the eventual star.

\subsection{StAR FORMATION}

Images of molecular clouds show that star formation is an active, ongoing process. The mechanisms that drive star formation must be efficient enough that such observations can be made, but it is also clear that it must be inefficient, considering the great majority of gas which is left behind in the surroundings 
(Larson, 2007). The rate of stellar production in the Galaxy is a puzzle, since it is much higher than the rate at which gas is returned back into the interstellar medium (ISM). This is suggestive of the irreversible effect of conversion of interstellar gas into stars (Knapp and Morris, 1985). There are still uncertainties concerning the conditions for which star formation is possible. The complexity of real star formation regions (e.g. magnetic fields, turbulence, rotation, geometry) makes numerical simulations a challenging task, and observations cannot reveal many details due to lack of resolution. Nevertheless, great advances have been made in this subject, and a simplified model is generally agreed upon to display the overall physics of star formation.

The densest cores within molecular clouds harbor the most ideal environment to trigger star formation. As mentioned previously, the dust shields the molecules from interstellar radiation fields (ISRF) and allows the molecules to form. The stability of the gravitationally bound core may be inferred from the virial theorem. The virial theorem evaluates the balance of energies of the core, by incorporating parameters such as mass, temperature, size, density, gravitational potential, and external pressure acting upon the core. Instability of the core initiates the first part of star formation evolution, which is free-fall collapse. During the free-fall phase, the core is assumed to be nearly isothermal. In an isothermal sphere, the density profile is expected to have the power-law $\rho \propto r^{-2}$. The core is thought to undergo an "inside-out" collapse where the collapse begins from inside and propagates outward (Young and Evans, 2005). At the inner radii, the density distribution is expected to be flatter, with a $-3 / 2$ power dependence. Fragmentation may produce smaller features as a result of a core reaching a limit called the Jean's mass-the minimum mass (and size) for a cloud to collapse self-gravitationally. This limit neglects the magnetic field, rotation, and turbulence, which are common ingredients to star formation. The Jean's mass is therefore only a simple approximation, and observations reveal the existence of factors supporting the clumps from collapse even when the observed clump masses exceed the Jean's limit (Shu et al., 1987). Fragmentation is supported observationally and also in simulations. Initial conditions of these simulations determine the limiting amount of fragmentation, showing that it is characteristic of the molecular cloud. During the contraction, the density and temperature within the core rise considerably, thus increasing the pressure. This pressure succeeds in slowing down the gravitational collapse, while the density and temperature continue to grow within the core, ending the first phase of collapse. After the first phase, the temperature climbs high enough for molecular hydrogen to 
dissociate, and the second phase of collapse sets in. The collapse turns from isothermal to adiabatic, and is eventually halted when the gravity can no longer overcome the pressure. When the collapse becomes adiabatic, the rate of collapse slows down significantly, and hydrostatic equilibrium is nearly reached in the core center. The resulting stable object in the central region is the earliest form of a star, referred to as a young stellar object (YSO) or protostar. YSOs represent the earliest stages before the star reaches the zero-age main sequence (ZAMS). This stage can be divided into several evolutionary sequences discussed in detail below: class -1, $0, \mathrm{I}, \mathrm{II}$, and III. Aided by rotation and the magnetic field, the circumstellar envelope is flattened into a disk, which accretes mass onto the protostars, advancing its growth as a star. Circumstellar disks are observed to be very common in star formation, although their physics is not completely understood. Observations of outflows and jets originating from cores support the presence of circumstellar disks. Jets and outflows involve magnetic fields, implying that the problem is magnetohydrodynamic. Magnetic fields and rotation are needed to produce jets in simulations (Banerjee and Pudritz, 2006; Machida et al., 2006). Simulations also show that jets and outflows are efficient in removing excess angular momentum.

In this simple, general model of initial star-formation, several factors are neglected in order to minimize the real complexity of star formation which is beyond the key focus of this thesis. These include the effects of magnetic fields, rotation of the cores, and likely deviation from spherical symmetry

\subsubsection{Young Stellar Objects}

The different classes of YSOs are classified by the spectral energy distribution (SED) over a range of different wavelengths. Class 0 is the first observable form of a protostar. In the preceding stage, class -1 , where the core is represented by a purely infalling embedded source, the central source cannot be observed due to the amount of dust and gas enshrouding it. To date, direct observational evidence of this stage has not been found. Class 0 sources are observed only as outflows; unipolar or bipolar. Outflows are an essential means of driving away angular momentum in the formation of the central star. At this stage, the central source can properly be labeled as a protostar since the mass of the envelope exceeds that of the star. For approximately $10^{4} \mathrm{yrs}$, the star remains hidden and undetected by wavelengths less than $100 \mu \mathrm{m}$, obscured by the surrounding dust and gas. It has been suggested that the short timescale of a YSO in class 0 is due to the observational difficulty of class 0 objects (Evans, 2003), and Visser et al. (2001, 2002) have proposed 
that class 0 YSOs are as frequent as class I YSOs, raising the timescale of class 0 sources by 10 times.

The source makes the transition from a class 0 to class I YSO when the sum of the star mass and disk mass exceed the envelope mass (Young and Evans, 2005). The following $10^{5}$ years after a star enters the class I stage, the source is finally observable up to the near-IR, but still invisible in the optical. The outflow continues but with less force than class 0 . The envelope material accretes onto the central star or is blown away by the outflow.

The source finally becomes optically apparent in the class II stage after the envelope material is virtually all lost (Young and Evans, 2005). The SED of a class II YSO is characterized by IR emission from the disk, and optical and UV emission from accretion. This stage is illustrated by the evolution of the circumstellar disk, commonly referred to as T Tauri stars. The circumstellar disks spark interest from the perspective of planet formation, and disk formation persists for up to $10^{6}$ years onwards.

The YSO spends the longest time $\left(10^{7}\right.$ years) contracting toward the main sequence as a class III source. In this stage, the disk may coagulate into a planetary system. As contraction continues, the temperature increases, moving the star horizontally leftward on the H-R diagram.

Depending on what stage the YSO is in, it will be observable only in select wavelengths ranging from far-IR to optical. Various molecules are used to trace these young objects hidden within dusty regions. Rotational and vibrational energy level transitions of these molecules are found in $\mathrm{mm}$ and sub-mm ranges, ideal for penetrating long columns of dust surrounding YSOs and for probing density and temperature information. Ultimately, the densities of $\mathrm{H}_{2}$ are the most fundamental in studying dense regions, since $\mathrm{H}_{2}$ is the most abundant molecule found in molecular clouds. $H_{2}$, however, cannot be directly detected, so different molecular tracers are used, assuming abundances relative to $\mathrm{H}_{2}$. Excitations of rotational energies are due to collisions of the tracer molecule with $\mathrm{H}_{2}$ or electrons, so these tracers essentially act as tools to exhibit the properties of molecular hydrogen.

The emission that is linked to specific transitions of molecular energy levels can be represented by some distribution of the molecular population when the density is greater than some critical density, at which spontaneous emission is surpassed by collisional excitation. This distribution is often approximated by the Boltzmann distribution, which can be estimated using the excitation temperature and the energy required for the transition. Local thermodynamic equilibrium (LTE) excitation is often assumed for the simplest 
approximations, and it is often a sufficient method. By observations of the densest molecular regions, it is possible to determine how likely the observed cores are of being embedded sources--the earliest stage of star formation-by deriving density, temperature, and mass estimates from the molecular tracers. It is debated, however, whether actual observations of purely infalling embedded sources-before the onset of outflows-in the far-IR has ever been observed, and such bold claims are often challenged (Moore et al., 1989; Schulz et al., 1991).

A molecular line profile of a cloud can uncover the structure of the region. One example of a commonly observed feature is a double-peak signature. There are two ways to interpret a double-peaked line, namely self-absorption and moving clumps. In the first case, if the observed region is enshrouded by a static spherical envelope, then the lines observed in optically thick gas will show a double-peaked line. This is due to absorption of radiation by the static envelope which blocks the central core. In the second case, the line may be displaying two separate velocity components (i.e., moving clumps). In order to rule out one interpretation, the region must be observed in one optically thick and one optically thin line. If both lines display the double peaked feature, then the presence of two sources with different velocities would be more likely. On the other hand, if the optically thick line shows a double peaked line with an observed dip, and the optically thin line displays a single-peak at the same velocity as the dip, then self-absorption may be justified.

In addition to structure, line profiles may also exhibit activities taking place in the region. One such possible activity is a cloud undergoing the process of "inside-out" collapse. For a simple case of a static spherical envelope surrounding a smaller spherical region which is experiencing infall, a line profile may be characterized by a blue-skewed double-peak feature with a self-absorption dip in the center (Evans, 2003). The stronger blue peak results from the back of the cloud, and the red peak from the front of the cloud. These characteristics can only be observed if the line is optically thick, but as discussed above, observations using an optically thin line must be carried out to rule out the presence of two separate velocity components. An ideal tool for this function is the pair of $\mathrm{HCO}^{+} J=3-2$ (optically thick) and $\mathrm{H}^{13} \mathrm{CO}^{+} \mathrm{J}=3-2$ (optically thin) lines. Although contributing factors (such as density and how much material still remains in the static envelope) may complicate the detection of infalling clouds, statistical studies have shown that inward motions are observed in excess (over outflow motions or random motions) within class $-1,0$, and I 
sources (Evans, 2003). The total number of sources in these studies may be insufficient, however, and the detection of red-skewed profiles among these sources is evidence that blue-skewed profiles are not universal.

\subsection{ORION B AND NGC 2024}

The Orion B giant molecular cloud is one of the most extensively-studied regions of active star formation. Also known as L1630, it is located at a distance of 415 pc (Anthony-Twarog, 1982), and this proximity has made the region an ideal place to study star-formation. Orion B is part of the larger Orion complex which includes Orion A to the south-west. It has been observed to extend out to $8^{\circ}$ in diffuse gas, but most of the gas is concentrated in two main sections: Orion B North and Orion B South. Orion B North contains star-forming regions NGC 2071 and NGC 2068, while Orion B South is home to NGC 2023, NGC 2024, and M78 (HH 19-27). Many studies have sought the identification of dense clumps within Orion $B$, to probe the distribution and properties of clumps and cores. Lada et al. (1991a) surveyed Orion B in CS and found that the emission is not distributed uniformly, but rather in a clumpy form (Fig. 1.1). 42 individual clumps were found, in various sizes, but in more elongated structures than round shapes. Virial masses were obtained for each clump, and it was found that at least $50 \%$ of the total clump mass is located in the five most massive cores mentioned above. Following this survey, Lada et al. (1991b) presented $2.2 \mu \mathrm{m}$ observations in Orion B. This study identified smaller features within the star-forming regions, and concluded that the majority of the detected sources were found in embedded clusters, which comprises only $\sim 18 \%$ of the entire surveyed region, suggesting that star formation within Orion B is a very localized process. More recently, Mitchell et al. (2001) identified 67 clumps in $850 \mu \mathrm{m}$ within Orion B South (shown in Fig. 1.3), and obtained more detailed information on clumps regarding their mass, temperature, and density.

One of the main sites of star-formation within Orion B is NGC 2024 in the South molecular cloud. NGC 2024 is a complex region which consists of several major components with observed features including an $\mathrm{H}$ II region, molecular ridge, dust lane, dense cores, and young star cluster. Fig. 1.2 shows the optical image of NGC 2024. The H II region - seen as an extensive, bright ionized region-is obscured by a dark band of dust, which appears to stem from the center bottom of the $\mathrm{H}$ II region. The unknown physical configuration of the entire system, specifically the relation between the molecular gas, dust, and the coincident $\mathbf{H}$ II region, 
has kept observers pursuing the cloud's structural and morphological information. Physical parameters including gas temperatures and densities can unlock clues to the actual star formation taking place in the dark obscured region.

\subsubsection{Previous STUdies}

The first studies of NGC 2024 date back to Ball et al. (1970) and Cesarsky (1971) where detections of $\mathrm{H} 137 \alpha(1.68 \mathrm{GHz})$ and $\mathrm{H} 157 \alpha(2.52 \mathrm{GHz})$ line emission from the region were published. Soifer and Hudson (1974) presented the first submillimeter map in $400 \mu \mathrm{m}$, followed by the $9 \mu \mathrm{m}$ map by Harper (1974) which gave color temperatures and grain models. Grasdalen (1974) discovered a bright IR point source in an $8.4 \mu \mathrm{m}$ map, also finding a large visual extinction toward the region. Mapping of NGC 2024 in IR emission was continued in the following years (Frey et al., 1979; Thronson et al., 1984), and spectroscopy in IR (Thompson et al., 1981; Black and Willner, 1984). The first molecular studies done in absorption included $\mathrm{OH}$ by Barnes et al. (1989) and formaldehyde $\left(\mathrm{H}_{2} \mathrm{CO}\right)$ by Crutcher et al. (1986). Molecular emission studies came later, including $\mathrm{H}_{2} \mathrm{CO}$ emission (Mangum and Wootten, 1993; van Dishoeck et al., 1993), $\mathrm{HCO}^{+}$(Barnes and Crutcher, 1990), $\mathrm{NH}_{3}$ (Schulz et al., 1991; Ho et al., 1993), CS (Schulz et al., 1991; Chandler and Carlstrom, 1996), and CO (Chandler and Carlstrom, 1996; Mitchell et al., 2001). The molecular studies have allowed for the modeling of the NGC 2024 spatial structure, putting together all the pieces of its components (e.g. molecular cloud, H II region, IR sources). After many attempts to seek the star responsible for the ionization of the H II region, Bik et al. (2003) identified the ionizing source of NGC 2024 to be a late $O$ type main sequence star, positioned directly east of the middle section of the ridge.

Some of the most notable studies of NGC 2024 were in sub-mm wavelengths by Mezger et al. (1988) and Mezger et al. (1992). These early dust observations of this cloud uncovered the presence of high-density condensations aligned in an elongated ridge, and they were claimed to be isothermal protostars in the earliest stages of protostellar evolution. Mezger et al. (1988; 1992) also presented temperature, density, and mass estimates, concluding that the cores were surprisingly cold and massive. The discovery of the FIR sources sparked further interest in this region and led to more sub-mm and mm observations. Schulz et al. (1991) closely inspected the FIR core positions with $C S$ and found few potential counterparts to the FIR cores, and presented temperatures, densities, and masses that strongly disagreed with those of Mezger 


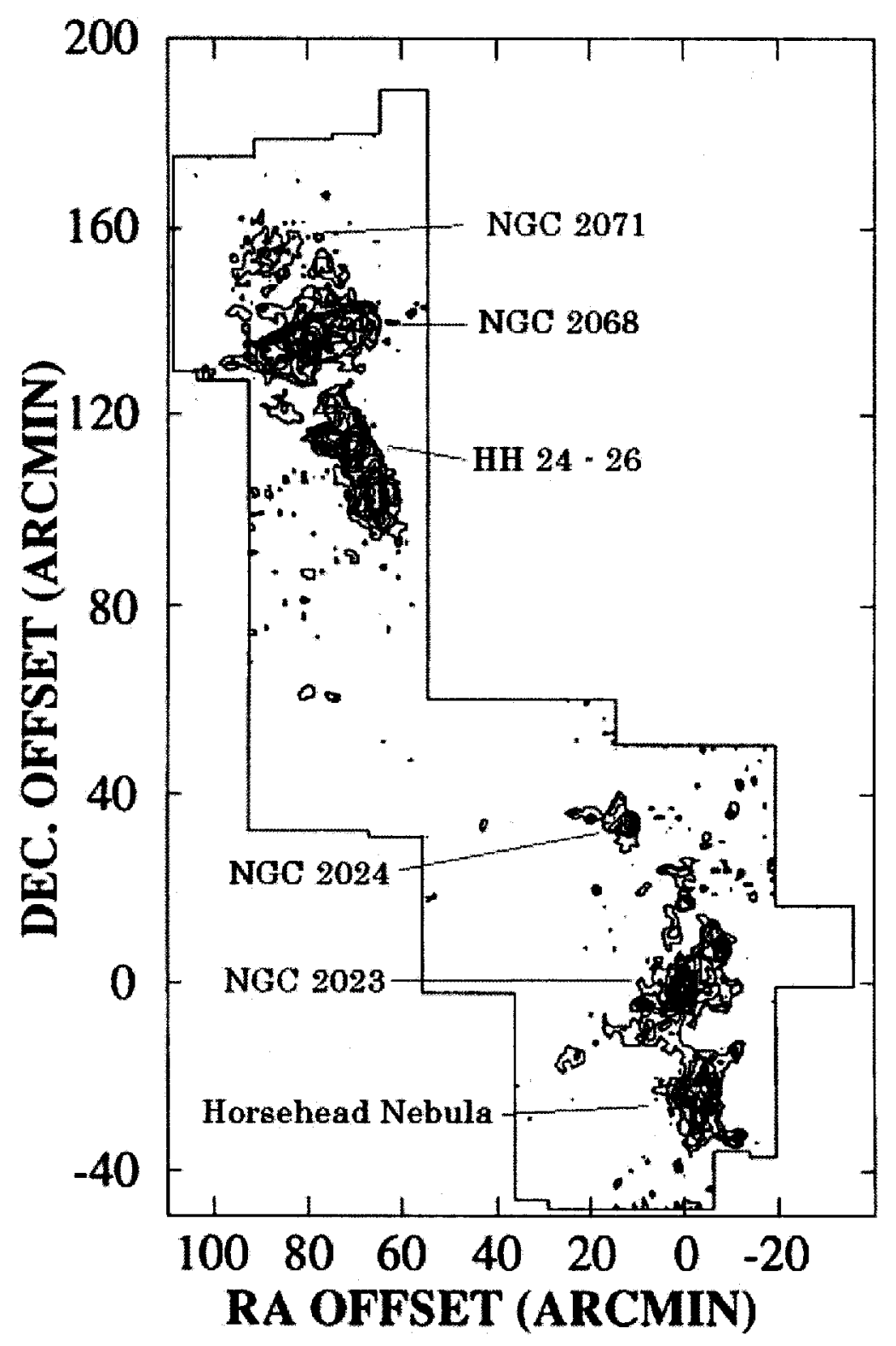

Figure 1.1: Dense molecular gas in Orion B seen in $C S(2-1)$ from Lada et al. (1991a). The three northernmost components make up Orion B North, and the three southern-most components make up Orion B South. 


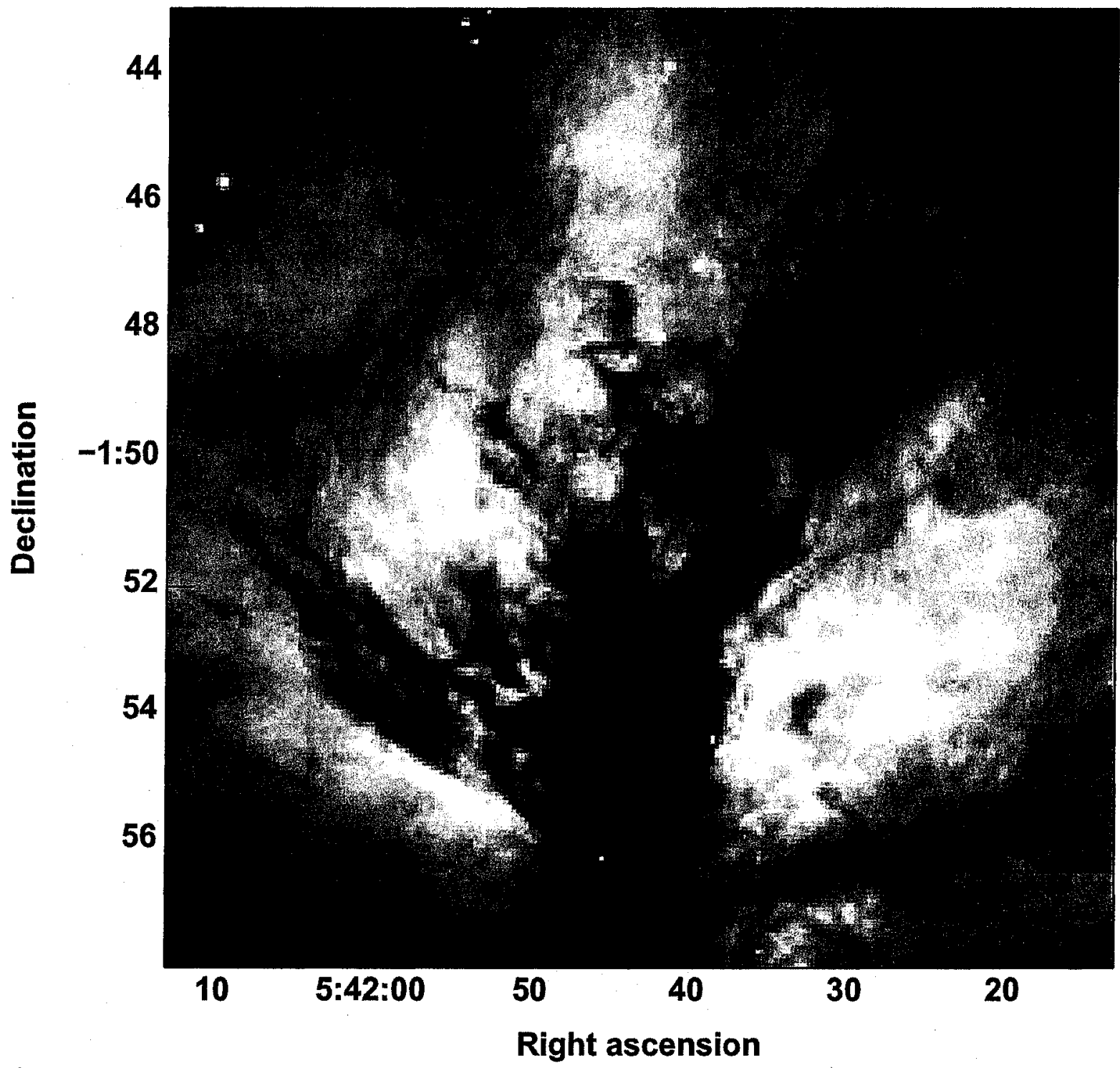

Figure 1.2: Optical image of NGC 2024 from the Digitized Sky Survey. The coordinates are displayed in epoch 2000. A dark lane of dust can be seen obscuring the center of the ionized region. 

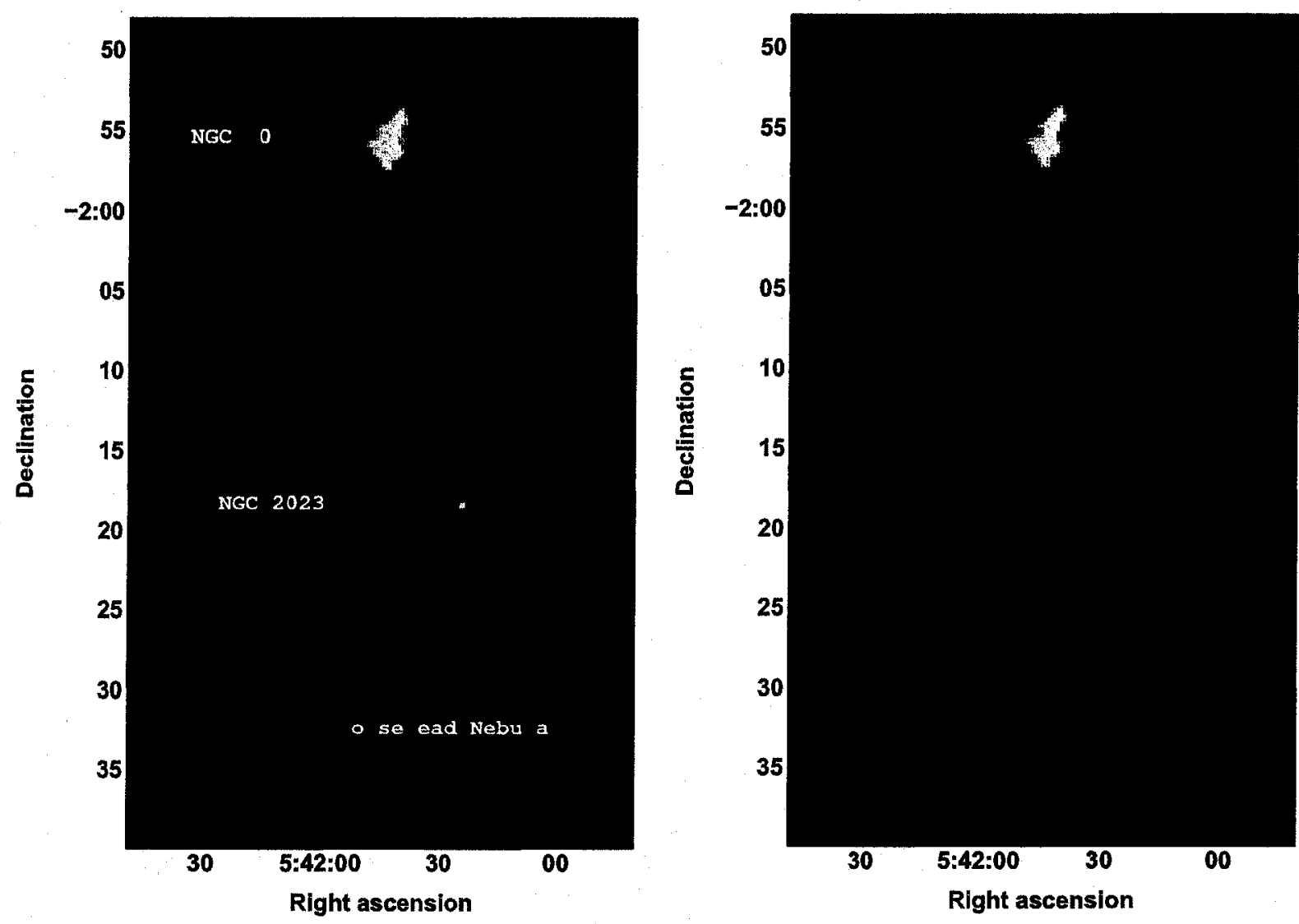

Figure 1.3: Dust continuum maps of Orion B south from SCUBA in $850 \mu \mathrm{m}$ (left) and $450 \mu \mathrm{m}$ (right), previously published in Johnstone et al. (2001), Mitchell et al. (2001), and Johnstone et al. (2006). The three major members of Orion B are labeled. The beam sizes are $15^{\prime \prime}$ for $850 \mu \mathrm{m}$ and $15^{\prime \prime}$ for $450 \mu \mathrm{m}$. 
et al. (1988; 1992). Visser et al. (1998) surveyed the FIR cores in sub-mm continuum at 450 and $800 \mu \mathrm{m}$ and compared images to molecular observations. More recently, the larger area of the Orion B molecular cloud has been observed at $850 \mu \mathrm{m}$ by Johnstone et al. (2001), Mitchell et al. (2001), and Johnstone et al. (2006) to probe the clump distribution in a larger area.

Further inspections of the FIR cores have revealed distinctive features of individual cores. Moore and Chandler (1989) suggested that FIR 4 contains a luminous stellar object observed in $2.2 \mu \mathrm{m}$, most likely a T Tauri star. FIR 4 is also associated with a near-IR reflection nebula and a unipolar, blue-shifted outflow lobe (Moore and Yamashita, 1995). Rodríguez et al. (2003) found compact $\mathrm{cm}$ sources in radio continuum at the positions of FIRs 4, 5, and 6, implying star formation. FIR 5 has an associated unipolar outflow (Richer et al., 1992), and a compact bipolar outflow in $C O$ has been observed at the position of FIR 6 (Richer, 1990). An $\mathrm{H}_{2} \mathrm{O}$ maser coincident with the position of FIR 6 was also found by Genzel and Downes (1977) before the detection of the FIR cores by Mezger et al. (1988). Wiesemeyer et al. (1997) observed FIRs 5 and 6 in $3 \mathrm{~mm}$ continuum and CS and resolved FIR 5 into two components, FIR 5-w and -e. This study also fit the SED of FIR 5-w and found that it is best modeled by a low bolometric luminosity source with a massive envelope. In $C S$, the authors could not find a close agreement in position with the FIR cores. In addition, other molecular studies of the FIR cores have also concluded a failure to detect coincident molecular peaks at the positions of Mezger's FIR cores. Mauersberger et al. (1992) found no CO counterparts to any of the FIR cores, and presented a disagreement between their detected $C S$ peaks and the dust cores in terms of position and size. The CS study of Schulz et al. (1991) also lacked agreement with the FIR cores. Wilson et al. (1995) also presented a CO study of NGC 2024, which resembled the features of the dust cores, but found a shift in the peak positions. These various molecular studies of the cores raise questions regarding their true protostellar nature. Some major concerns are the discrepancies between the dust core and the molecular core positions, the vast range of derived core properties-namely temperature and mass - and the features of the region which appear to change with the observed molecule or frequency. The disagreement seen within the previous studies makes it a challenge to put together an evolutionary picture of each core which satisfies all observations. With improvements in resolution and instrument sensitivity, the interpretation of these cores should become more transparent, and the details of their evolution should surface. Detailed information associating FIRs 1-3, and 7 to unique features and 
possible star-formation has not yet been made available.

\subsection{Physical PRoperties: TEMPERATURE AND DENSITY}

\subsubsection{TEMPERATURE OF FIR CORES}

Probing the physical conditions of the FIR cores can provide more insight of the system as a whole. Most importantly, the temperatures of the individual cores offer information about what stage of star-formation the region is in. Because ranges in temperature can be traced to a specific phase of star-formation, this parameter is crucial in the evaluation of the observations, and an inaccurate evaluation could lead to misinterpretations. Since the discovery of the FIR cores by Mezger et al. (1988), kinetic temperatures and number densities have been derived for the FIR cores using different molecular tracers and models. These numerous past studies vary, however, in their results, revealing still a need for further investigations.

Mezger et al. (1988) used their observations of the cores and found that the integrated emission could be fit with three temperature components. Their density analysis showed that the majority of the emission comes from the cold dust component, having temperature as low as $16 \mathrm{~K}$. From this temperature and by comparison of computed evolutionary tracks of pre-main sequence stars, the authors suggest that the discovered cores were isothermal protostars without luminous stellar cores.

The paper was immediately followed by contradicting studies. In the subsequent years, several groups presented evidence that some of the cores were associated with embedded stellar activity. A $2.2 \mu \mathrm{m} \mathrm{IR}$ source was found in coincidence with FIR 4 by Moore and Chandler (1989), and a compact CO outflow centered on FIR 6 was reported by Richer (1990). FIR 5 was also predicted to be associated with a CO outflow (Richer et al., 1989; Richer, 1990; Barnes and Crutcher, 1990). With this evidence, further investigations of core temperatures were carried out, challenging the claims of Mezger et al. (1988). Moore et al. (1989) used a CS line to map the region of NGC 2024. They found a lower limit of $28 \mathrm{~K}$ for the emission, using LTE. They showed that the continuum spectrum of Mezger et al. (1988) could be fit with a single component temperature of $47 \mathrm{~K}$ by modifying the dust emissivity slightly. A similar result was presented by Schulz et al. (1991), who mapped the region in $N_{3}$ inversion lines and transitions of $C S$. Their $\mathrm{NH}_{3}$ observations suggested a kinetic temperature of $35 \mathrm{~K}$, and from $\mathrm{CS}$ a range between 35 and 45 
Table 1.1: Summary of Temperature Results from Previous Studies

\begin{tabular}{llll}
\hline \hline Study & Observation & Temperature & Comments \\
\hline Mezger et al. (1988) & FIR & $>16 \mathrm{~K}$ & cold dust/gas component \\
Moore et al. (1989) & $\mathrm{CO}$ transition & $>28 \mathrm{~K}$ & LTE \\
Shultz et al. (1991) & $\mathrm{NH}_{3} \& \mathrm{CS}$ & $35-45 \mathrm{~K}$ & LVG \\
Lis et al. (1991) & $\mathrm{HCO}^{+}$emission & $>27 \mathrm{~K}$ & \\
Mezger et al. (1992) & $1300 \mu \mathrm{m} \& 870 \mu \mathrm{m}$ & $>19 \mathrm{~K}$ & cold dust/gas component \\
Gaume et al. (1992) & $\mathrm{NH}_{3}$ & $>20 \mathrm{~K}$ & uncertainties not clarified \\
Mauersberger et al. (1992) & $\mathrm{C}^{18} \mathrm{O}, \mathrm{C}^{17} \mathrm{O}, \mathrm{L}^{13} \mathrm{C}^{18} \mathrm{O}$ & $35-40 \mathrm{~K}$ & \\
Ho et al. (1993) & $\mathrm{NH}_{3}$ & $40 \mathrm{~K}$ & FIRs 5-7 \\
Graf et al. (1993) & $\mathrm{CO}$ transitions & $67 \mathrm{~K}$ & FIR 5 \\
\hline
\end{tabular}

K using the large velocity gradient (LVG) models - similar to LTE. LVG models simplify the complexities of radiative transfer by assuming that a small region is not affected by the radiation approaching from large distances. They emphasized the lack of evidence for a cool emission component in their data, and found that the low temperature component was not required to fit the continuum data of Mezger et al. (1988). These authors ruled out the likeliness that the cores were protostars, claiming that the clumps resemble more young stellar objects with embedded heat sources. Similar to Moore et al. (1989), Lis et al. (1991) found a lower limit of $27 \mathrm{~K}$ using $\mathrm{HCO}^{+}$emission. Mezger et al. (1992) presented a follow-up to their first paper, with the addition of new observations at 1300 and $870 \mu \mathrm{m}$. They again found that their data fit a three component model, but their cold gas component of $16 \mathrm{~K}$ was raised to $19 \mathrm{~K}$. Gaume et al. (1992) found temperatures resembling Mezger et al. (1992) from VLA observations of $\mathrm{NH}_{3}$, where FIRs 1-3, and 6 had $\mathrm{T}<\sim 20 \mathrm{~K}$, and FIRs 4 and 5 with $\mathrm{T}>20 \mathrm{~K}$. It is worth mentioning that the temperatures may be highly uncertain, since their spectra have very low signal-to-noise ratios, and no error analysis is presented. Mauersberger et al. (1992) found kinetic temperatures in the range of 35 to $40 \mathrm{~K}$ using line ratios of spectra of $C^{18} \mathrm{O}, \mathrm{C}^{17} \mathrm{O}$, and ${ }^{13} \mathrm{C}^{18} \mathrm{O}$ transitions. Ho et al. (1993) obtained the same $\mathrm{NH}_{3}$ lines as Gaume et al. (1992) with the VLA but found different temperatures, with FIRs 5-7 having $40 \mathrm{~K}$, whereas Gaume et al. (1992) found $20 \mathrm{~K}$ for FIR 6. Graf et al. (1993) used $C O$ transitions to specifically observe FIR 5. They dispute with the results of Mezger et al. (1992), reporting that the bulk of the gas has a temperature of 67 $\mathrm{K}$, and that the cold gas makes up a much smaller percentage of the entire region. A summary of FIR core temperatures derived in previous studies is tabulated in Table 1.1. 


\subsubsection{FORMALDEHYDE AS TEMPERATURE PROBE}

Kinetic temperatures and number densities have been derived for the FIR cores using various methods, one of which is formaldehyde line ratios. Mangum and Wootten (1993) showed how temperature and density information can be obtained from the ratios of observed formaldehyde transition intensities. Using spherical LVG models, Mangum and Wooten presented the temperature- and density-dependent relations that specific ratios offer, making it possible to derive accurate estimates of temperature and density. Using this method, Mangum et al. (1999) derived approximate temperature estimates independent of gas density effects for FIRs 3-7. Formaldehyde line ratios can also be used to derive temperatures assuming LTE. Since the relative population occupying two states are related through the Boltzmann equation by the temperature, a few assumptions can be made to find a simple relationship for the temperature. Mangum and Wootten (1993) showed that in LTE and with additional assumptions, the temperature can be approximated as a function of the observed intensity ratio of the two levels and the difference in the energies. One of the advantages of using formaldehyde as a temperature and density probe is that several transitions can be measured with the same telescope-including a few that may be detected in the same spectrum--therefore minimizing calibration uncertainties. Formaldehyde is also an ideal tool to probe high-density regions and, unlike some other tracers of temperature, formaldehyde is relatively high in abundance and does not vary significantly within the interstellar medium (Mangum and Wootten, 1993).

Measurements of density and temperature variations throughout the cloud-especially across the FIR cores and from one FIR core to another-would contribute to a more complete picture of the structure of NGC 2024. Temperature differences between the cores and the surrounding regions could be indicating clues as to why the peaks in dust do not coincide with the molecular peaks. In isolated regions, significantly higher temperatures within cores compared to its surroundings would hint at the presence of embedded sources in more advanced stages of star-formation, whereas cooler temperatures would imply prestellar sources. In a clustered region, however, the interpretation is more complicated, with the contribution of core-heating due to the radiation from nearby cluster stars. The true influence of clustered regions on star formation is not well-understood. Overcoming the obstacles of studying such complicated regions will shed light on how they vary from (or resemble) isolated regions. Hence, a large map of the NGC 2024 region 
in a temperature/density tracing molecule is required to make accurate assessments of the nature of the

ridge and its dense cores. An emphasis should be made on cuts through the FIR core positions to study variations and differences from the core with its surroundings.

\subsection{Thesis CONTENT}

In order to explore the temperature and density variation throughout the ridge of NGC 2024, observations were carried out at the James Clerk Maxwell telescope (JCMT) in 2004 in four $\mathrm{H}_{2} \mathrm{CO}$ transitions. These transitions-namely, $3_{03} \rightarrow 2_{02}, 3_{22} \rightarrow 2_{21}, 5_{05} \rightarrow 4_{04}$, and $5_{23} \rightarrow 4_{22}$-were chosen to permit measurements of temperature and density. Details concerning the method of observations, and the properties of the transitions are discussed in Chapter 2. The ratios of $H_{2} C O$ transitions $3_{03} \rightarrow 2_{02} / 3_{22} \rightarrow 2_{21}$ and $5_{05} \rightarrow$ $4_{04} / 5_{23} \rightarrow 4_{22}$ give reliable kinetic temperatures, and the density is obtained from the $\mathrm{H}_{2} \mathrm{CO}$ transition ratio of $3_{03} \rightarrow 2_{02} / 5_{05} \rightarrow 4_{04}$. For the formaldehyde transitions of $3_{03} \rightarrow 2_{02}, 3_{22} \rightarrow 2_{21}$, and $5_{05} \rightarrow 4_{04}$, maps are produced from the observations and used to evaluate the temperature and density structure inside

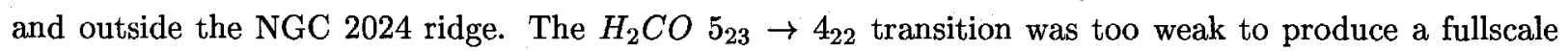
map, but the observations at the FIR core positions and its immediate surroundings are still beneficial in the temperature and density evaluations. The formaldehyde maps and spectra are introduced in Chapter 2, and the derived temperatures and densities with an emphasis on the FIR core positions are presented in Chapter 3. Chapter 3 also combines the temperature and density information obtained from $\mathrm{H}_{2} \mathrm{CO}$ and used with various other observations (e.g., sub-mm, mid-IR), to obtain information such as clump masses, column densities, and $\mathrm{H}_{2} \mathrm{CO}$ fractional abundance. Chapter 4 discusses these results and their implications. Comparisons are made between the formaldehyde maps and a number of other observations to probe the nature of the FIR cores. Lastly, the virial theorem is used to determine whether the cores are contracting.

Observations of NGC 2024 in $\mathrm{HCO}^{+}$and $\mathrm{H}^{13} \mathrm{CO}^{+}$were obtained from the JCMT as well, with the intention of detecting infall at the FIR core positions. In Section 3.3, these observations are used to derive optical depths and excitation temperatures at various positions, and also to calculate the column densities. As with $\mathrm{H}_{2} \mathrm{CO}$, the $\mathrm{HCO}^{+}$column densities are used to derive $\mathrm{HCO}^{+}$fractional abundance at the FIR cores, which are presented in Section 3.5. 


\section{OBSERVATIONS}

All observations were carried out between August 2004 and December 2004 at the James Clerk Maxwell telescope on Mauna Kea, in queue observing mode, using the facility A-band and B-band receivers. Four formaldehyde lines were observed, namely $\mathrm{H}_{2} \mathrm{CO}_{303} \rightarrow 2_{02}$ at $218.2222 \mathrm{GHz}, \mathrm{H}_{2} \mathrm{CO} 3_{22} \rightarrow 2_{21}$ at 218.4756 $\mathrm{GHz}, \mathrm{H}_{2} \mathrm{CO} 5_{05} \rightarrow 4_{04}$ at $362.7360 \mathrm{GHz}$, and $\mathrm{H}_{2} \mathrm{CO}_{523} \rightarrow 4_{22}$ at $365.3634 \mathrm{GHz}$. The details of these transitions are presented in Table 2.1. Because of the quantum properties of formaldehyde transitions and its assymetric structure and rotational characteristics, some transition ratios are sensitive to temperature, while some are sensitive to density (Mangum and Wootten, 1993). The intensity ratios $3_{03} \rightarrow 2_{02} / 3_{22} \rightarrow 2_{21}$ and $5_{05} \rightarrow 4_{04} / 5_{23} \rightarrow 4_{22}$ provide two independent measures of the gas kinetic temperature. The intensity ratio $3_{03} \rightarrow 2_{02} / 5_{05} \rightarrow 4_{04}$ provide a value of the total gas density [i.e. $\mathbf{n}\left(H_{2}\right)$ ]. Because of their proximity in frequency, it was possible to obtain the $3_{03} \rightarrow 2_{02}$ and $3_{22} \rightarrow 2_{21}$ lines in a single tuning. The $3_{03} \rightarrow$ $2_{02} / 3_{22} \rightarrow 2_{21}$ intensity ratio is, therefore, independent of calibration uncertainties.

A number of raster maps was made in the $218 \mathrm{GHz}$ line pair, using a $7.5^{\prime \prime}$ grid (i.e., a third of a beamwidth) and an integration time of 5 seconds at each position. Line intensities were obtained as antenna temperatures, $T_{A}^{*}$, corrected for atmospheric and telescope losses. The total integration time was calculated so as to achieve a final rms of $0.1 \mathrm{~K}$ in $T_{A}^{*}$. Raster maps in the $362 \mathrm{GHz}$ line were taken with a $5^{\prime \prime}$ grid (again a third of a beam) and a $5^{\prime \prime}$ integration time per position. Because the $365 \mathrm{GHz}$ line is weaker, raster-mapping was not attempted. Instead, $\mathrm{n} \times 1$ grid maps (i.e., cuts at fixed declination) through each FIR source position were obtained, where $n=5$ for FIR 1 through FIR 5 and $n=3$ for FIR 6 and FIR 7 .

Table 2.1: Summary of Observed Formaldehyde Transitions

\begin{tabular}{ccccc}
\hline \hline $\begin{array}{c}\text { Transition } \\
{\left[J_{\left.K_{a} K_{c}\right]}\right.}\end{array}$ & $\begin{array}{c}\text { Frequency } \\
{[G H z]}\end{array}$ & $\begin{array}{c}\text { Beam size } \\
{\left[{ }^{\prime \prime}\right]}\end{array}$ & $\begin{array}{c}E_{u}{ }^{a} \\
{[K]}\end{array}$ & $S^{b}$ \\
\hline $3_{03} \rightarrow 2_{02}$ & 218.2222 & 23 & 23 & 2.9994 \\
$3_{22} \rightarrow 2_{21}$ & 218.4756 & 23 & 68 & 1.6667 \\
$5_{05} \rightarrow 4_{04}$ & 362.7360 & 15 & 52.4 & 4.9971 \\
$5_{23} \rightarrow 4_{22}$ & 365.3634 & 15 & 99.7 & 4.1998 \\
\hline
\end{tabular}

\footnotetext{
${ }^{a}$ Upper energy

${ }^{b}$ Line strength
} 
Table 2.2: Positions of FIR Sources from Mezger et al. (1992)

\begin{tabular}{l|lc}
\hline \hline Source & R.A. (J2000) & Declination (J2000) \\
\hline FIR 1 & $05^{h} 39^{m} 09^{s} 9$ & $-1^{\circ} 55^{\prime} 17^{\prime \prime}$ \\
FIR 2 & $05^{h} 39^{m} 11^{8} 0$ & $-1^{\circ} 55^{\prime} 34^{\prime \prime}$ \\
FIR 3 & $05^{h} 39^{m} 11 . .^{s} 4$ & $-1^{\circ} 55^{\prime} 52^{\prime \prime}$ \\
FIR 4 & $05^{h} 39^{m} 12 .^{s} 6$ & $-1^{\circ} 56^{\prime} 13^{\prime \prime}$ \\
FIR 5 & $05^{h} 39^{m} 13 . .^{8} 0$ & $-1^{\circ} 57^{\prime} 08^{\prime \prime}$ \\
FIR 6 & $05^{h} 39^{m} 13 . .^{s} 7$ & $-1^{\circ} 57^{\prime} 30^{\prime \prime}$ \\
FIR 7 & $05^{h} 39^{m} 13 . .^{s} 5$ & $-1^{\circ} 57^{\prime} 50^{\prime \prime}$ \\
\hline
\end{tabular}

Table 2.3: Summary of Observed $\mathrm{HCO}^{+}$and $\mathrm{H}^{13} \mathrm{CO}^{+}$Lines

\begin{tabular}{ccc}
\hline \hline Transition & $\begin{array}{c}\text { Frequency } \\
{[\mathrm{GHz}]}\end{array}$ & $\begin{array}{c}\text { Beam size } \\
{\left[{ }^{\prime \prime}\right]}\end{array}$ \\
\hline $\mathrm{H}^{13} \mathrm{CO}^{+} J=3-2$ & 260.2555 & 21 \\
$\mathrm{HCO}^{+} J=3-2$ & 267.5576 & 21 \\
\hline
\end{tabular}

Table 2.2 shows the right ascension and declination coordinates used for the seven FIR cores.

Spectra of $\mathrm{HCO}^{+} \mathrm{J}=3-2$ at $267.5576 \mathrm{GHz}$ and $\mathrm{H}^{13} \mathrm{CO}^{+} J=3-2$ at $260.2555 \mathrm{GHz}$ were obtained at each of the seven FIR positions. The details of these transitions are shown in Table 2.3. Integration times at each position were 5 minutes for the $\mathrm{HCO}^{+}$line and 10 minutes for the $\mathrm{H}^{13} \mathrm{CO}^{+}$line.

All spectral data were reduced using SPECX, a spectral data reduction package which allows the transformation of raster map observations into a map which can be viewed in user-specified axes and integrated intensities. To make each of the three $\mathrm{H}_{2} \mathrm{CO}$ transition maps (i.e. $3_{03} \rightarrow 2_{02}, 3_{22} \rightarrow 2_{21}$, and $5_{05} \rightarrow 4_{04}$ ), the spectra within the raster-map were inspected and baseline-corrected. If a bad spectrum appeared, it was omitted from the map. Each spectrum was incorporated in the final map as a single cell which corresponds to the position of the observed data. Spectra of the same position were combined and averaged to give a better signal-to-noise ratio. The final map is in the form of a data cube, with axes of frequency, velocity, and intensity, and may be viewed in various formats within SPECX. To study the velocity, the map can be viewed at specified ranges of velocities. The line strength is recorded as the antenna temperature $T_{A}^{*}$. To obtain temperature and density information, the intensity is required to be in the form of radiation temperature $T_{R}$. Radiation temperatures were derived from the antenna temperature by correcting for the main-beam efficiency $\eta_{M B}$. This efficiency has been derived for Mars, Jupiter, and Uranus and is offered at 
the JCMT website ${ }^{1}$. Fig. 2.9 shows several sample spectra after the baseline has been removed. In these spectra, the antenna temperature $T_{A}^{*}$ is plotted as a function of velocity.

\section{$2.1 \quad \mathrm{H}_{2} \mathrm{CO} 3_{03} \rightarrow 2_{02}$ AND $3_{22} \rightarrow 2_{21}$ MAPS}

The linear baseline was first removed from each spectrum of the $3_{03} \rightarrow 2_{02}$ and $3_{22} \rightarrow 2_{21}$ lines. Out of the 1023 channels in each spectrum, 200 on each side were dropped. A map was made for each observed night, with cell sizes of $7.5^{\prime \prime}$ by $7.5^{\prime \prime}$. The maps of the North region on 4 different nights were averaged into one final North map. The South consists of three small maps and one medium map. The overlapping regions were averaged to make one final South map. The final South and North maps were combined to make the final map of the entire region. The final map is centered on RA $5^{h} 39^{m} 12 .^{s} 4$ and $\operatorname{dec}-1^{\circ} 56^{\prime} 25 .^{\prime \prime} 31$, with the RA offsets ranging from $-120^{\prime \prime}$ to $+120^{\prime \prime}$, and the declination from $-120^{\prime \prime}$ to $+150^{\prime \prime}$.

The final averaged map of the $3_{03} \rightarrow 2_{02}$ line is presented in Fig. 2.1, where the intensity has been integrated from 8 to $13 \mathrm{~km} \mathrm{~s}^{-1}$. The high intensity emission can be seen as an elongated ridge that is concentrated in the north-south direction, slightly tilted to the west. The ridge is clearly composed of two components: a northern clump and a southern clump. The northern clump is more elongated, and stretches further south in a fainter emission. The southern clump is slightly stronger, and less extended than the northern clump. The elongated ridge is separated by a gap of faint emission. The diffuse emission extends out to $50^{\prime \prime}$ from the central peaks. The $3_{22} \rightarrow 2_{21}$ map also shown in Fig. 2.2 displays a weaker emission, but with the same features as the $3_{03} \rightarrow 2_{02}$ map. The emission maintains the same elongated north-south orientation, with the southern clump having a stronger intensity. The channel maps shown in Fig. 2.3 exhibit the velocity difference along the entire ridge. By plotting the map at intervals of $0.5 \mathrm{~km}$ $\mathrm{s}^{-1}$, it is clear that the northern clump peaks at a lower velocity than the south. At $9 \mathrm{~km} \mathrm{~s}^{-1}$, only the northern region is faintly visible, and there is nothing apparent in the south. As the velocity is increased, the north becomes stronger, while the south begins to appear, but by $10.5 \mathrm{~km} \mathrm{~s}^{-1}$, the north region fails to be detected. The south emission faintly lingers until $12.5 \mathrm{~km} \mathrm{~s}^{-1}$, by which no sign of the north emission is traced. The maps are used to calculate the physical properties (e.g. temperature, number density, column

\footnotetext{
${ }^{1} \mathrm{JCMT}$ beam efficiencies webpage http://www.jach.hawaii.edu/JCMT/spectral_line/Standards/beameff.html
} 
density) at various positions and are presented in the remaining sections of this chapter. The implications of the results-including the derived physical properties and observed velocity features-are discussed in detail in the next chapter. A detailed discussion comparing the $\mathrm{H}_{2} \mathrm{CO}$ maps with previous observations and proposing the nature of the FIR cores within the ridge is also presented.

\section{$2.2 \mathrm{H}_{2} \mathrm{CO} 5_{05} \rightarrow 4_{04} \mathrm{MAP}$}

For the spectra of the $\mathrm{H}_{2} \mathrm{CO} 5_{05} \rightarrow 4_{04}$ transition at $362.7360 \mathrm{GHz}$, a similar method was employed to create a map of the entire ridge. From each spectrum, 30 channels on each end were dropped to leave a total of 963 channels. The three sets of observations from the different nights have three different map sizes. The overlapping regions were averaged to make one final map, with cell sizes of $5^{\prime \prime}$ by $5^{\prime \prime}$. The offsets range from $-90^{\prime \prime}$ to $+90^{\prime \prime}$ in RA, and $-142.5^{\prime \prime}$ to $150^{\prime \prime}$ in declination. Fig. 2.4 shows the integrated intensity map of the $5_{05} \rightarrow 4_{04}$ line. The intensity appears weaker than the $3_{03} \rightarrow 2_{02}$ line, but in general, no obvious differences can be observed. The ridge is divided into the north and south, separated by a gap of very weak intensity. The southern clump peaks appear stronger, with a less elongated structure than the north. The channel maps in Fig. 2.5 follow the same velocity trend as seen in the $3_{03} \rightarrow 2_{02}$ channel map. The north emission dominates at velocities from 9 to $10.5 \mathrm{~km} \mathrm{~s}^{-1}$, but is taken over by the south emission at higher velocities, where the north emission fades away completely. A comparison of the $3_{03} \rightarrow 2_{02}$ transition map and the $5_{05} \rightarrow 4_{04}$ transition map is presented in the overplot of Fig. 2.6. The figure shows the two maps are very similar in their extent, shape, and peak positions. As with the maps from the $3_{03} \rightarrow 2_{02}$ and $3_{22} \rightarrow 2_{21}$ transitions, this map will also contribute to the calculations of physical properties in the following sections, and the implications of the results are discussed in the following chapter.

\section{3 $\mathrm{H}_{2} \mathrm{CO} 5_{23} \rightarrow 4_{22}$ SPECTRA}

The $\mathrm{H}_{2} \mathrm{CO}_{5} \mathrm{5}_{23} \rightarrow 4_{22}$ line at $365.3634 \mathrm{GHz}$ is weaker in comparison with other $\mathrm{H}_{2} \mathrm{CO}$ lines, so a single cut across each FIR was obtained at a fixed declination. For FIRs 1 through 5, 5-point cuts were taken, with each cut centered on the FIR position, extending out to $30^{\prime \prime}$ on each side. For FIRs 6 and 7, 3-point cuts were taken centered on the FIR position, extending out to $15^{\prime \prime}$ on each side. The spectra of the cuts 


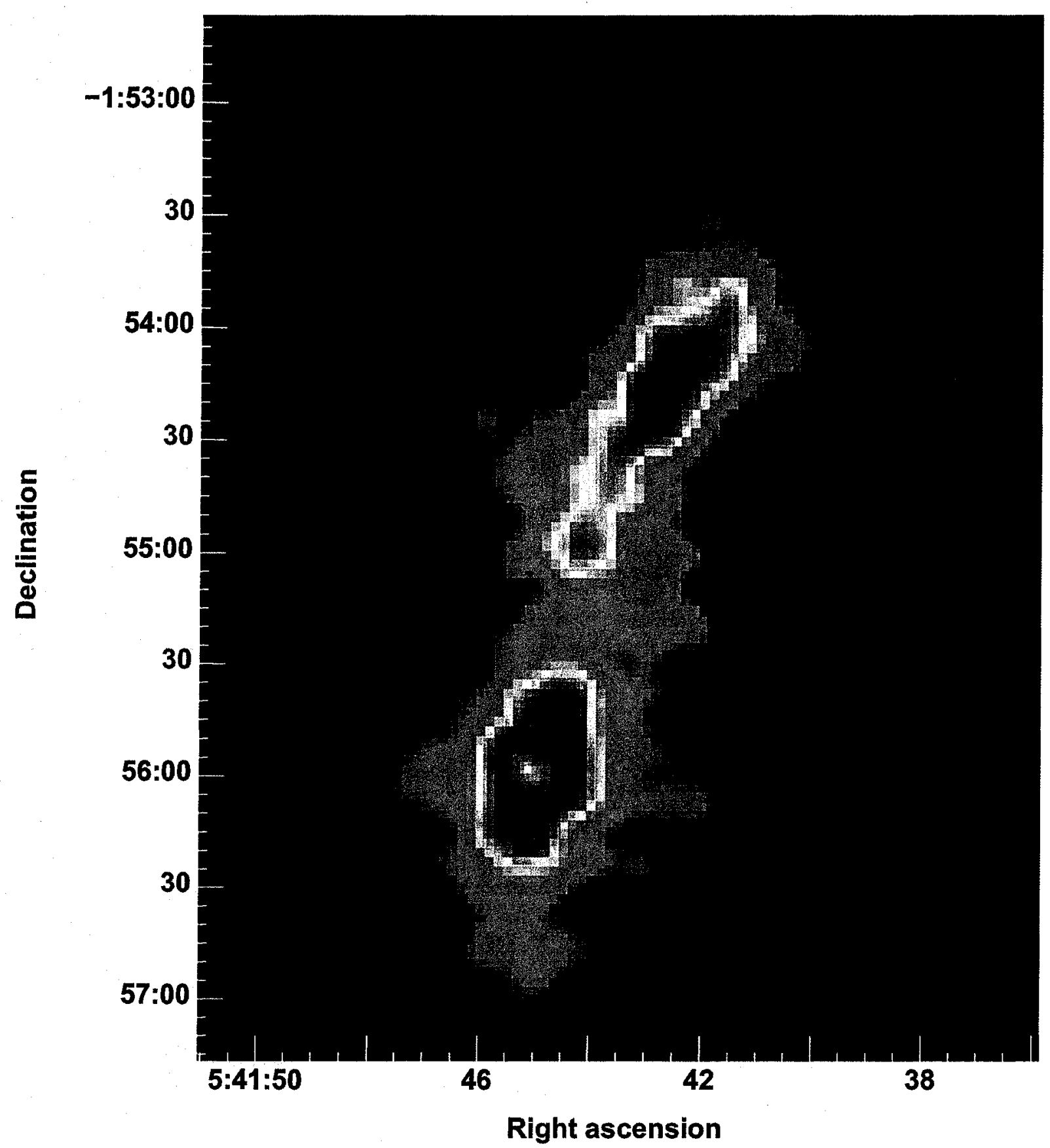

Figure 2.1: Integrated intensity map of NGC 2024 ridge observed in $\mathrm{H}_{2} \mathrm{CO} 3_{03} \rightarrow 2_{02}$ line at 218.2222 $\mathrm{GHz}$. The intensity is integrated over the velocity range of $8-13 \mathrm{~km} \mathrm{~s}^{-1}$. The maximum value is at $12 \mathrm{~K} \mathrm{~km} \mathrm{~s}^{-1}$, and the minimum is at $0 \mathrm{~K} \mathrm{~km} \mathrm{~s}^{-1}$. The beam size is $23^{\prime \prime}$. The coordinates are in epoch 2000 , as are all the subsequent figures unless otherwise stated. 


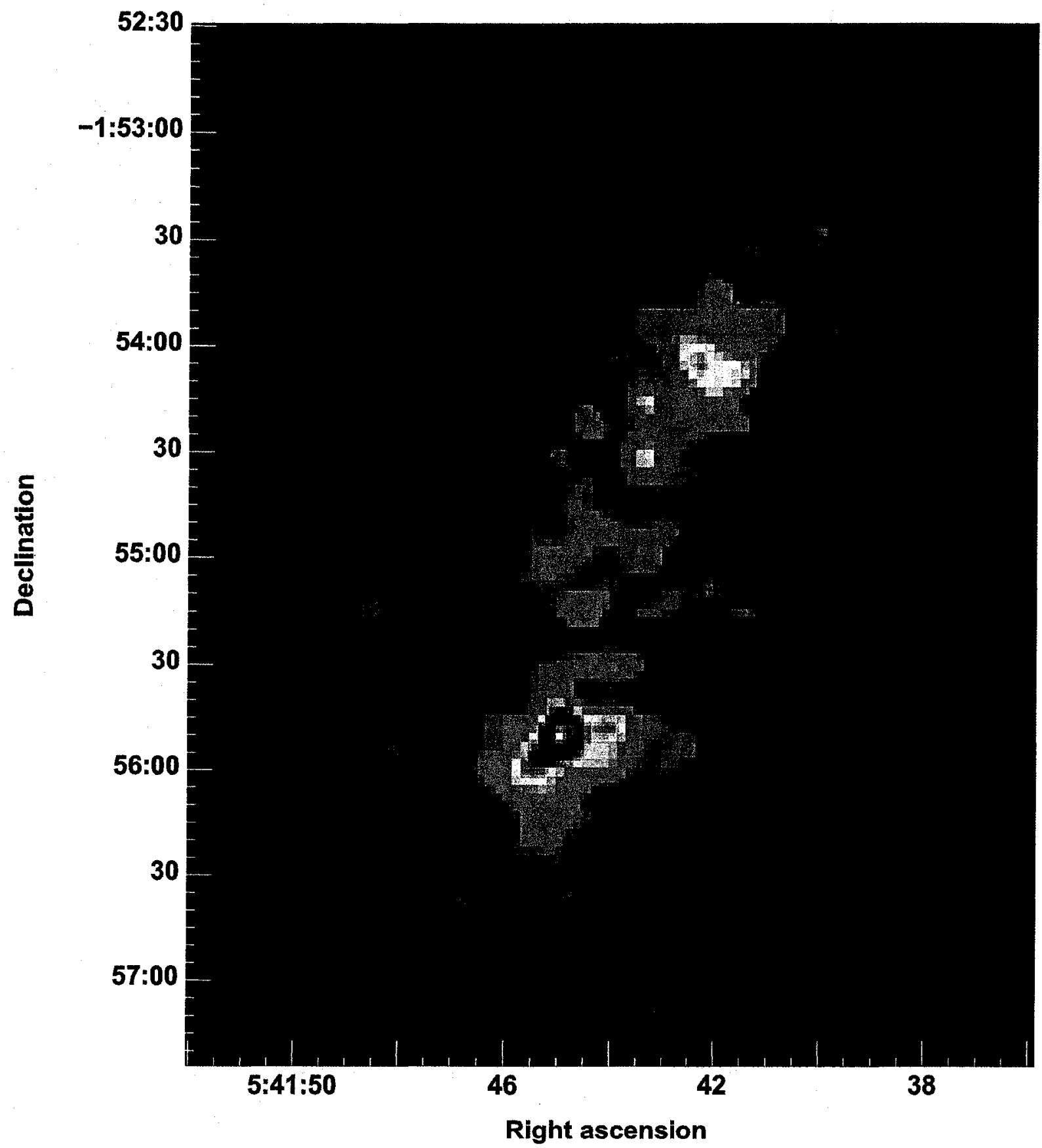

Figure 2.2: Integrated intensity map of NGC 2024 ridge observed in $\mathrm{H}_{2} \mathrm{CO} 3_{22} \rightarrow 2_{21}$ line at 218.4756 $\mathrm{GHz}$. The intensity is integrated over the velocity range of $8-13 \mathrm{~km} \mathrm{~s}^{-1}$. The maximum value is at $5 \mathrm{~K} \mathrm{~km} \mathrm{~s}^{-1}$, and the minimum is at $0 \mathrm{~K} \mathrm{~km} \mathrm{~s}^{-1}$. The beam size is $23^{\prime \prime}$. 

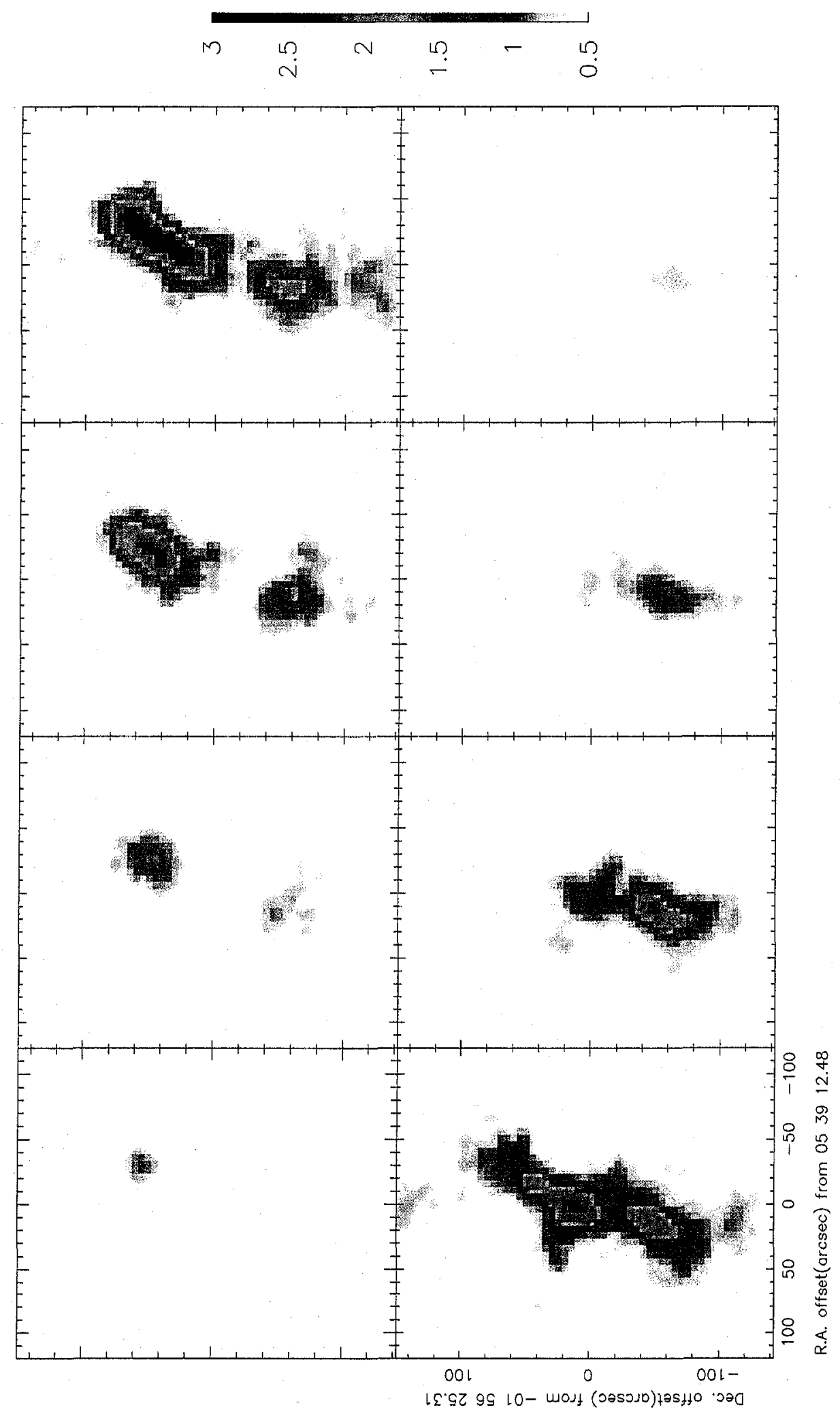

Figure 2.3: Channel maps of $\mathrm{H}_{2} \mathrm{CO}_{303} \rightarrow 2_{02}$ transition. The velocity range is from 9 to $12.5 \mathrm{~km} \mathrm{~s}^{-1}$, taken at intervals of $0.5 \mathrm{~km} \mathrm{~s}^{-1}$. 


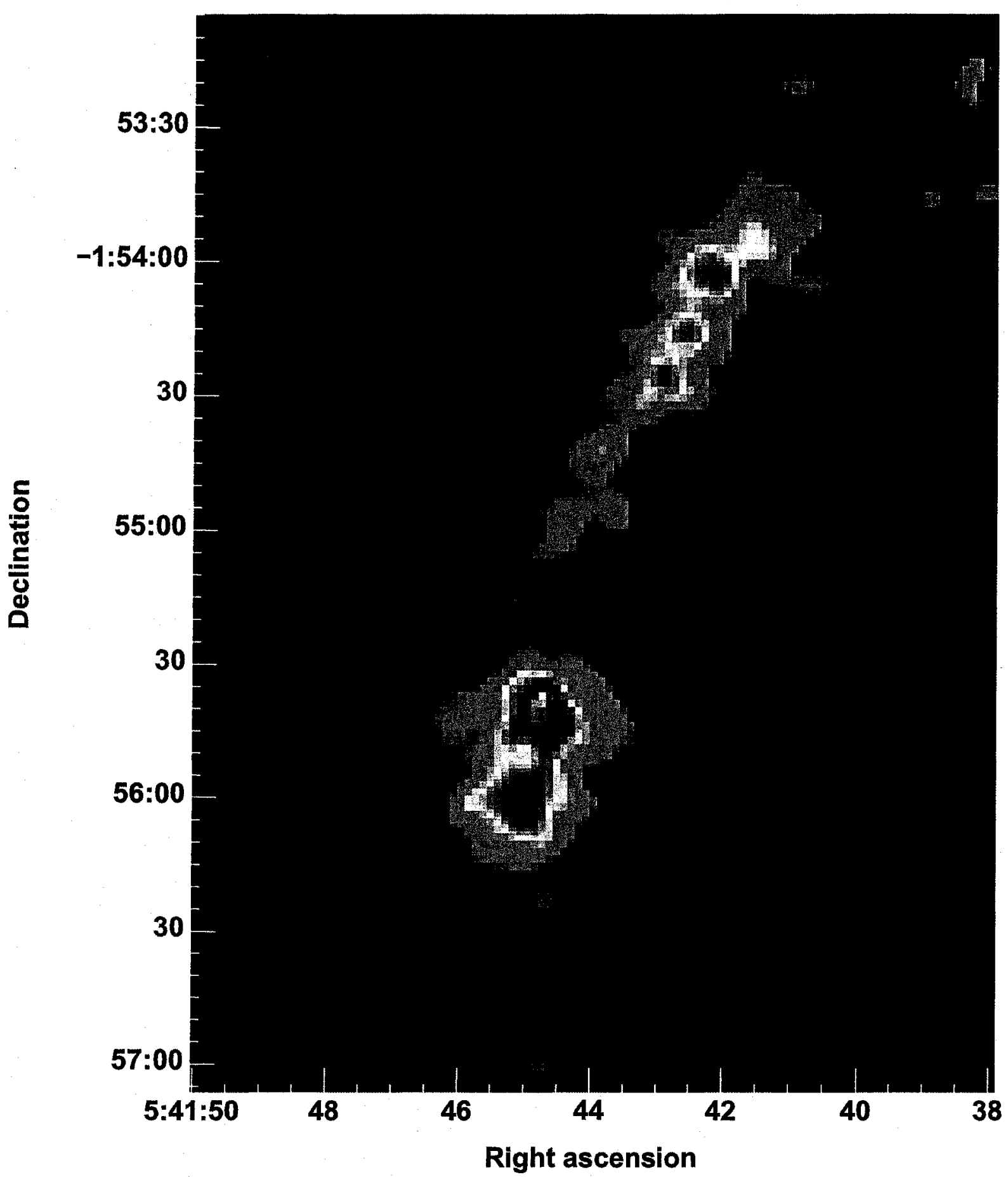

Figure 2.4: Integrated intensity map of NGC 2024 ridge observed in $\mathrm{H}_{2} \mathrm{CO} 5_{05} \rightarrow 4_{04}$ line at 362.7360 $\mathrm{GHz}$. The intensity is integrated over the velocity range of $8-13 \mathrm{~km} \mathrm{~s}^{-1}$. The maximum value is at $10.4 \mathrm{~K} \mathrm{~km} \mathrm{~s}^{-1}$, and the minimum is at $0 \mathrm{~K} \mathrm{~km} \mathrm{~s}^{-1}$. The beam size is $15^{\prime \prime}$. 


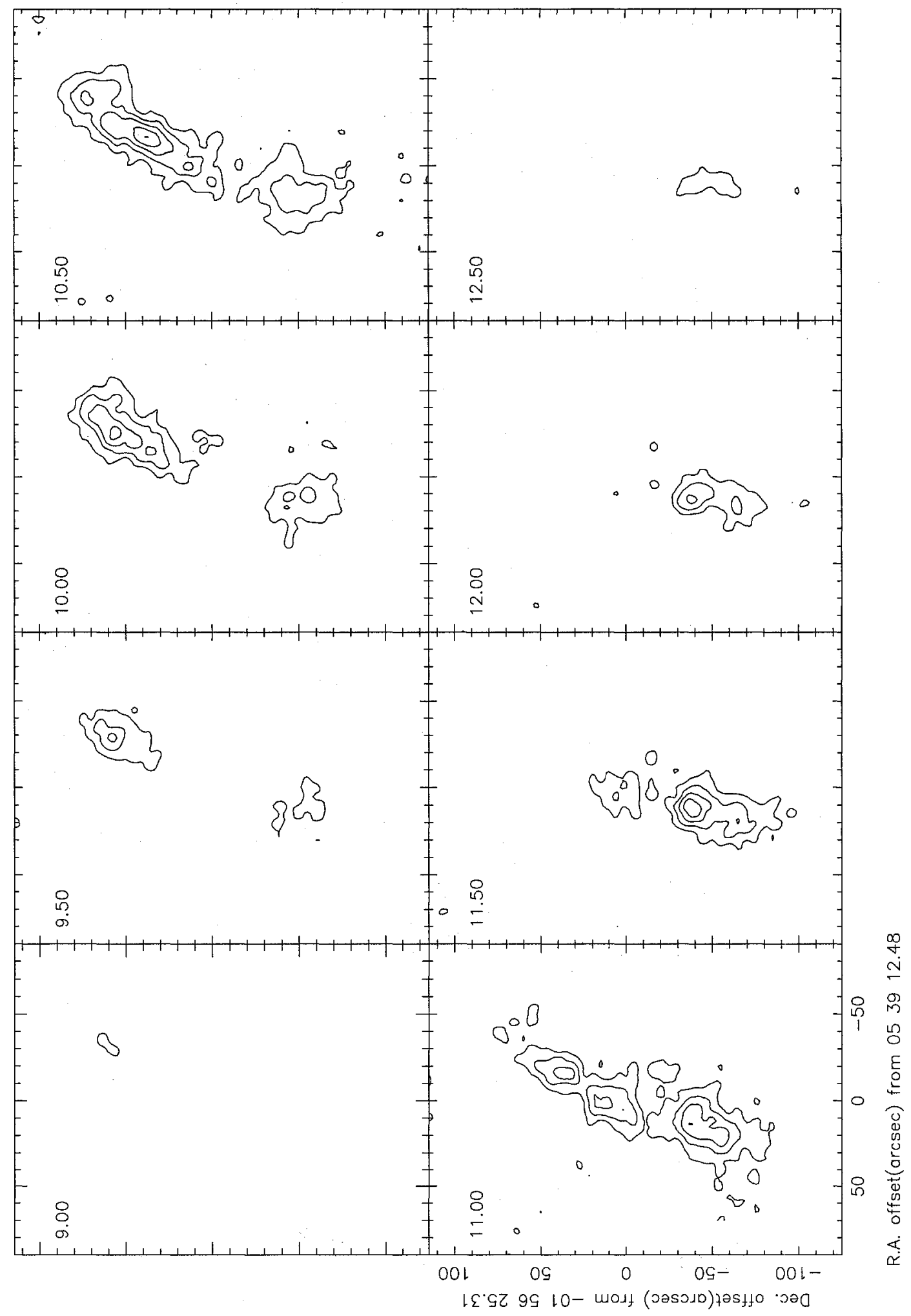

Figure 2.5: Contour channel maps of $\mathrm{H}_{2} \mathrm{CO} 5_{05} \rightarrow 4_{04}$ transition. The velocity range is from 9 to $12.5 \mathrm{~km}$ $\mathrm{s}^{-1}$ at intervals of $0.5 \mathrm{~km} \mathrm{~s}^{-1}$. 


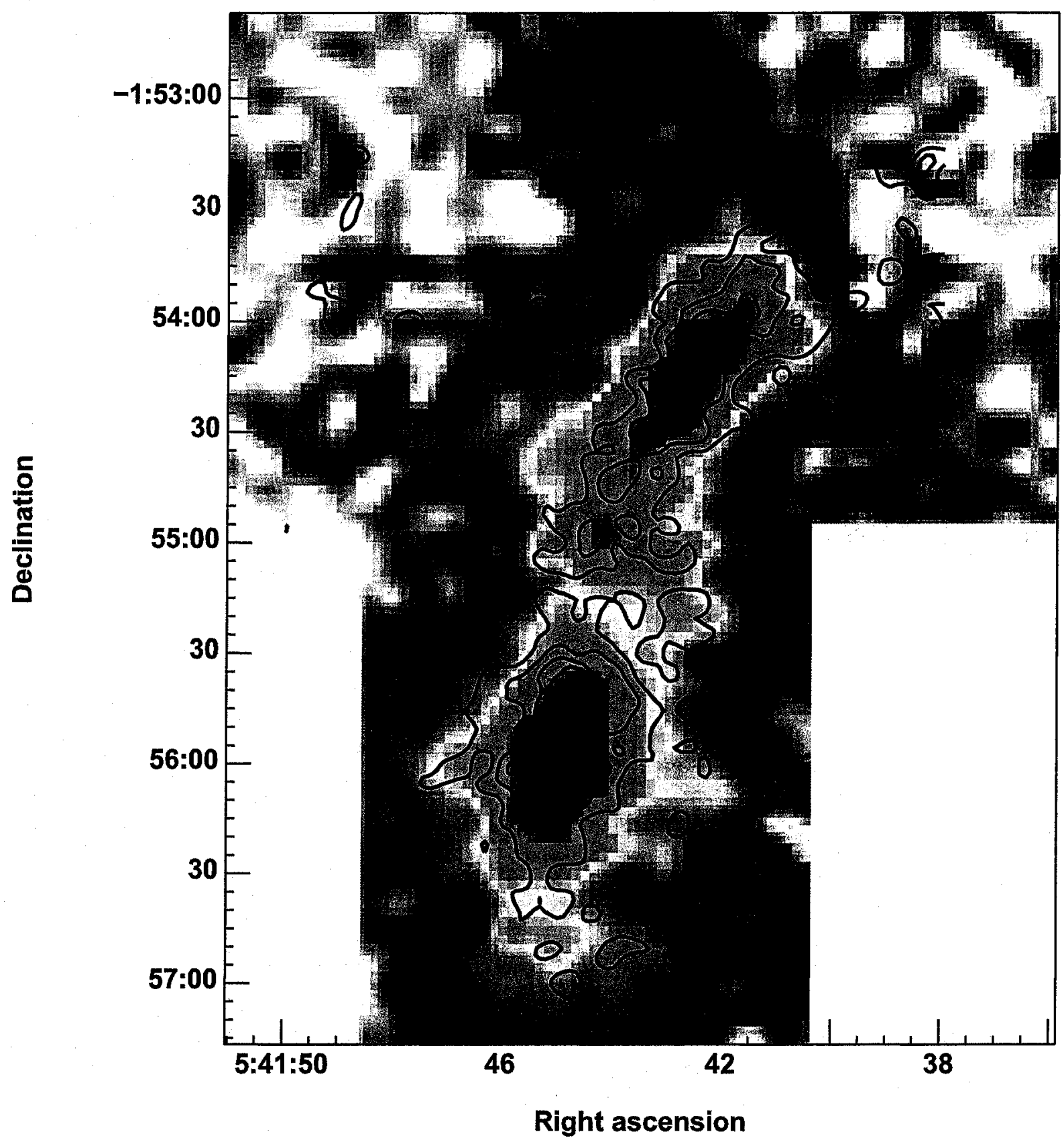

Figure 2.6: Contours of $\mathrm{H}_{2} \mathrm{CO} 5_{05} \rightarrow 4_{04}$ transition map plotted over $3_{03} \rightarrow 2_{02}$ transition map. The contours show intensities at $2,4,6,8$, and $10 \mathrm{~K} \mathrm{~km} \mathrm{~s}^{-1}$. 


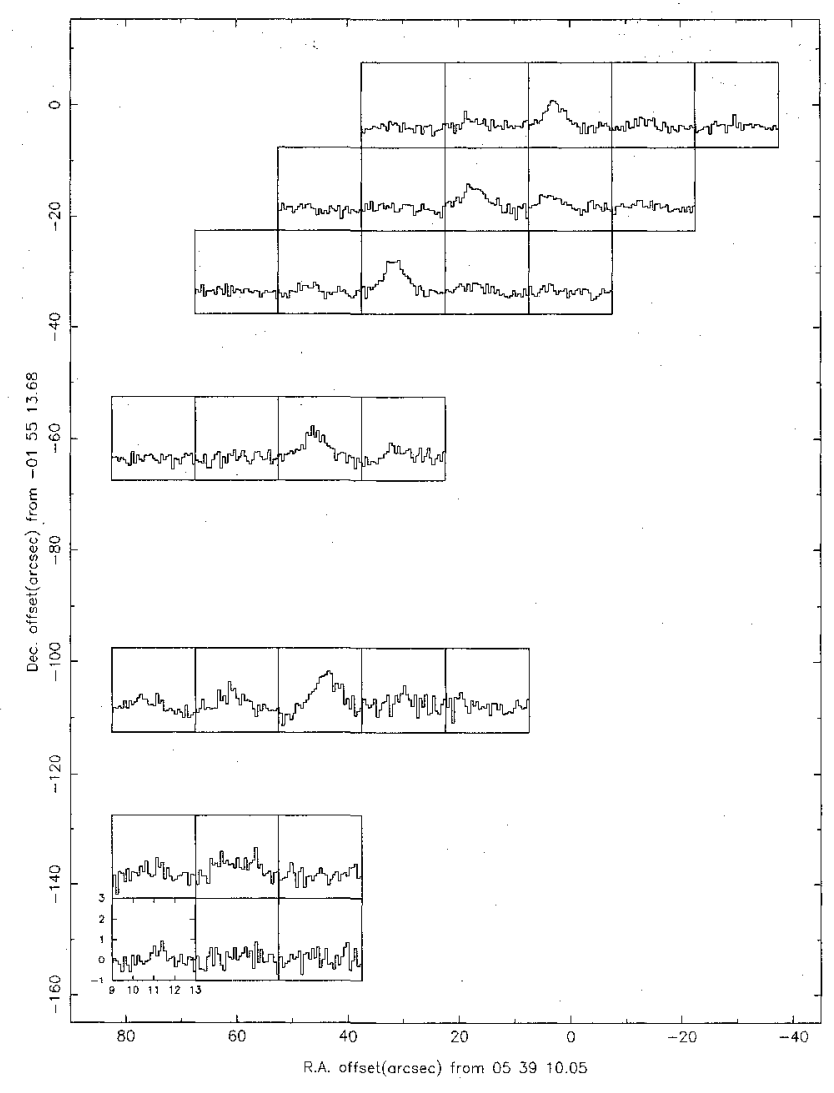

Figure 2.7: Grid map of $\mathrm{H}_{2} \mathrm{CO} 5_{23} \rightarrow 4_{22}$ transition at constant-declination cuts through FIR core positions. The position of each spectrum in the grid corresponds to its true position within NGC 2024.

are shown at their corresponding positions in Fig. 2.7. Since the positions of $5_{23} \rightarrow 4_{22}$ line observations are limited, a map could not be produced, but the cuts are used to derive temperatures at the most vital positions. The results are presented in section 3.1 in the following chapter.

\subsection{1 $\mathrm{HCO}^{+} J=3-2$ AND $\mathrm{H}^{13} \mathrm{CO}^{+} J=3-2$ SPECTRA}

The $\mathrm{HCO}^{+} J=3-2$ and $\mathrm{H}^{13} \mathrm{CO}^{+} J=3-2$ lines at frequencies 267.5576 and $260.2555 \mathrm{GHz}$ were taken at each FIR core position. Each spectrum was corrected for its baseline by fitting it to a polynomial baseline and removing the best fit. When more than one spectrum was available for a single position, the average was taken. Fig. 2.8 shows the 267.5576 and $260.2555 \mathrm{GHz}$ lines for the FIR core positions stacked in order of declination. The general trend of the northern FIR cores having a lower velocity than the southern cores in formaldehyde lines is seen here again. In both lines, the southern peaks have velocities that peak about 1 

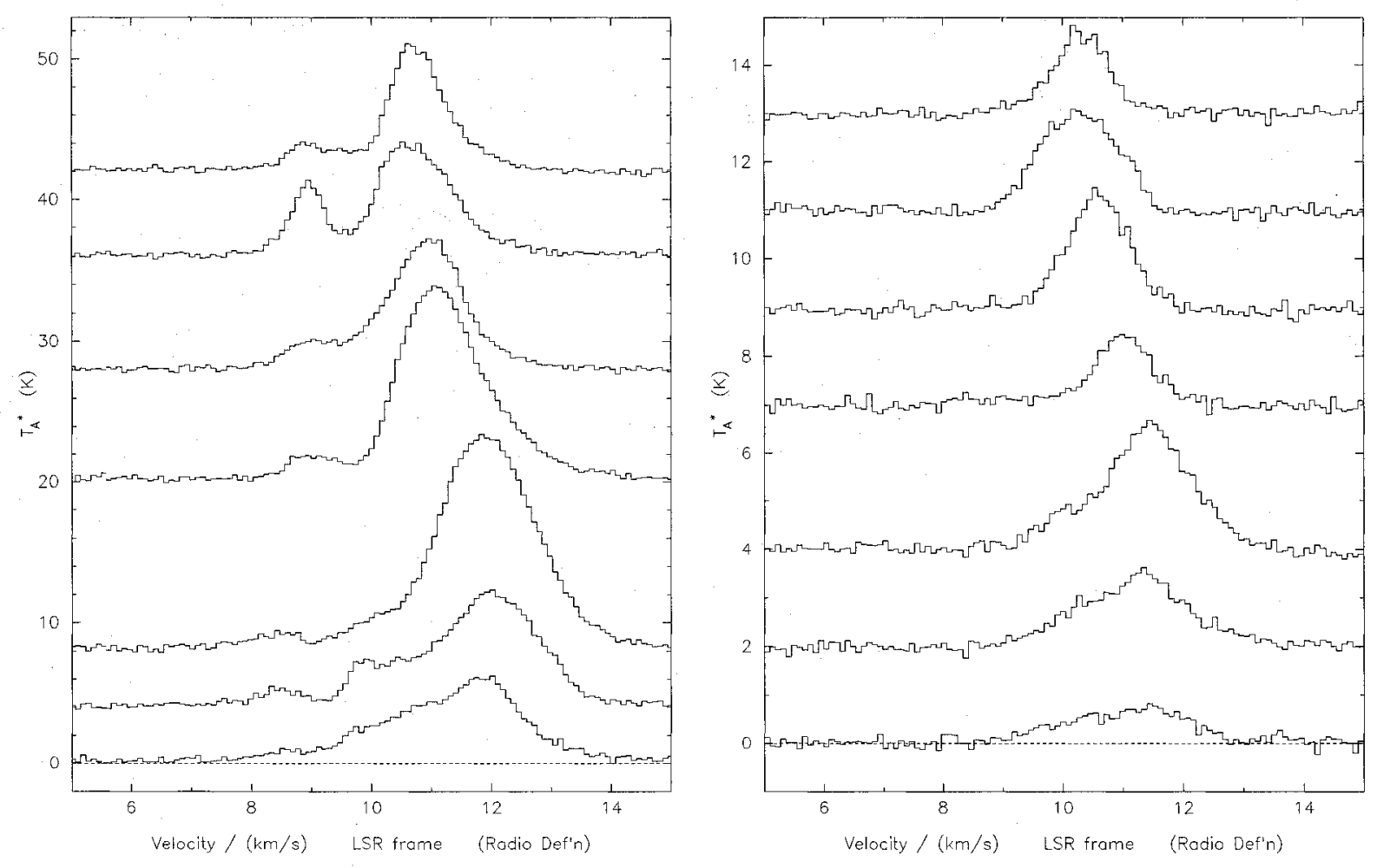

Figure 2.8: Spectra of $\mathrm{HCO}^{+} J=3-2$ line at $267.5576 \mathrm{GHz}$ (left) and the $\mathrm{H}^{13} \mathrm{CO}^{+} J=3-2$ line at $260.2555 \mathrm{GHz}$ at FIR core positions. The intensities are offset to display all the spectra simultaneously. The FIR positions are in order from FIR 1 (at top) to FIR 7 (at bottom). Note the difference in the $\mathrm{y}$-axis scales between the two plots.

$\mathrm{km} \mathrm{s}^{-1}$ greater than the northern FIR cores. The peaks of the FIR core positions in both lines are observed at the same velocities for each FIR core, but they also exhibit major differences. The intensities of the $\mathrm{HCO}^{+}$lines are stronger than the $\mathrm{H}^{13} \mathrm{CO}^{+}$lines. Also, the shapes of the peaks are vastly different between the two transitions. The $\mathrm{HCO}^{+}$lines show double-peak features, which is most prominent in FIR 2. This feature will be further discussed in Section 3.3 in the next chapter and in more detail in the discussion chapter.

\subsection{CUtS Through FIR CORE POSITIONS}

The FIR core positions in the $\mathrm{H}_{2} \mathrm{CO}$ transitions $3_{03} \rightarrow 2_{02}$ and $3_{22} \rightarrow 2_{21}$ are further examined by studying the gradients across cuts centered on the FIR core positions. The cuts are made so that the RA offsets 

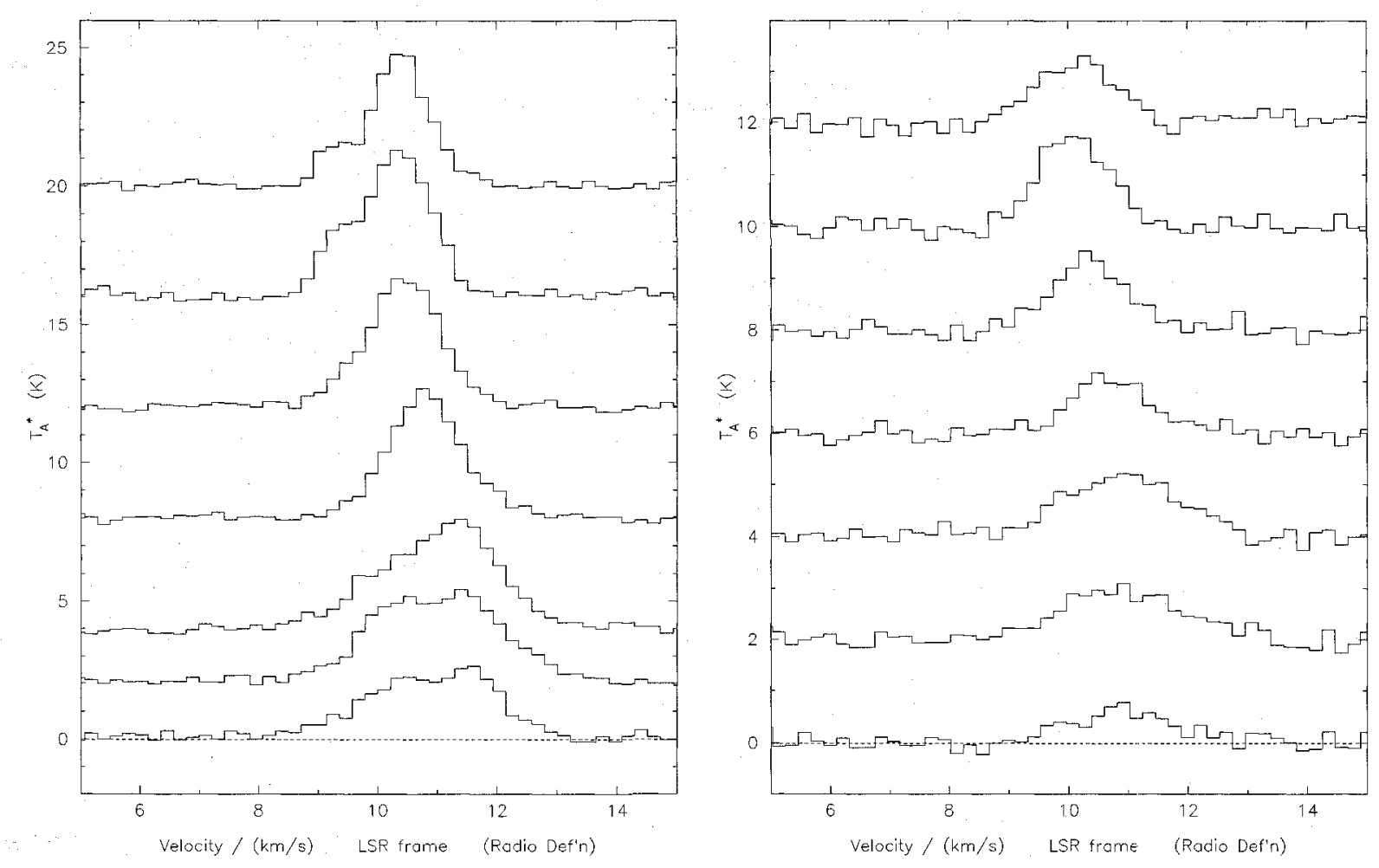

Figure 2.9: Spectra of $\mathrm{H}_{2} \mathrm{CO}_{303} \rightarrow 2_{02}$ line (left) and $3_{22} \rightarrow 2_{21}$ line (right) at FIR core positions: FIRs 1-7 from top to bottom. Note the difference in the y-axis scales between the two plots.

ranged from $-45^{\prime \prime}$ to $45^{\prime \prime}$ on either side of each FIR core position. In order to obtain a better signal-to-noise ratio, the spectra within the cuts were averaged over squares of 9 cells ( 3 by 3 cells). Similarly, the FIR cuts of the $5_{05} \rightarrow 4_{04}$ transition were averaged over 5 cells like the $5_{23} \rightarrow 4_{22}$ cuts. The measured integrated intensities of the averaged cuts are given in Table 2.4. Figs. 2.9-2.10 show the FIR core positions stacked vertically for the four different formaldehyde lines, separated by offsets that do not necessarily represent spatial distances. A common trend among all the lines is that the FIR cores positioned in the southern clump have higher velocity peaks compared to the cores in the northern clump. This is also observed in the channel maps presented in Figs.2.3 and 2.5. The peak velocity for each FIR core remains about the same for all transitions. As previously mentioned, the $3_{03} \rightarrow 2_{02}$ and $5_{05} \rightarrow 4_{04}$ lines are stronger than the $3_{22} \rightarrow 2_{21}$ and $5_{23} \rightarrow 4_{22}$ lines. The velocity information of these spectra at FIR positions is discussed in section 4.4.1 of the next chapter. 

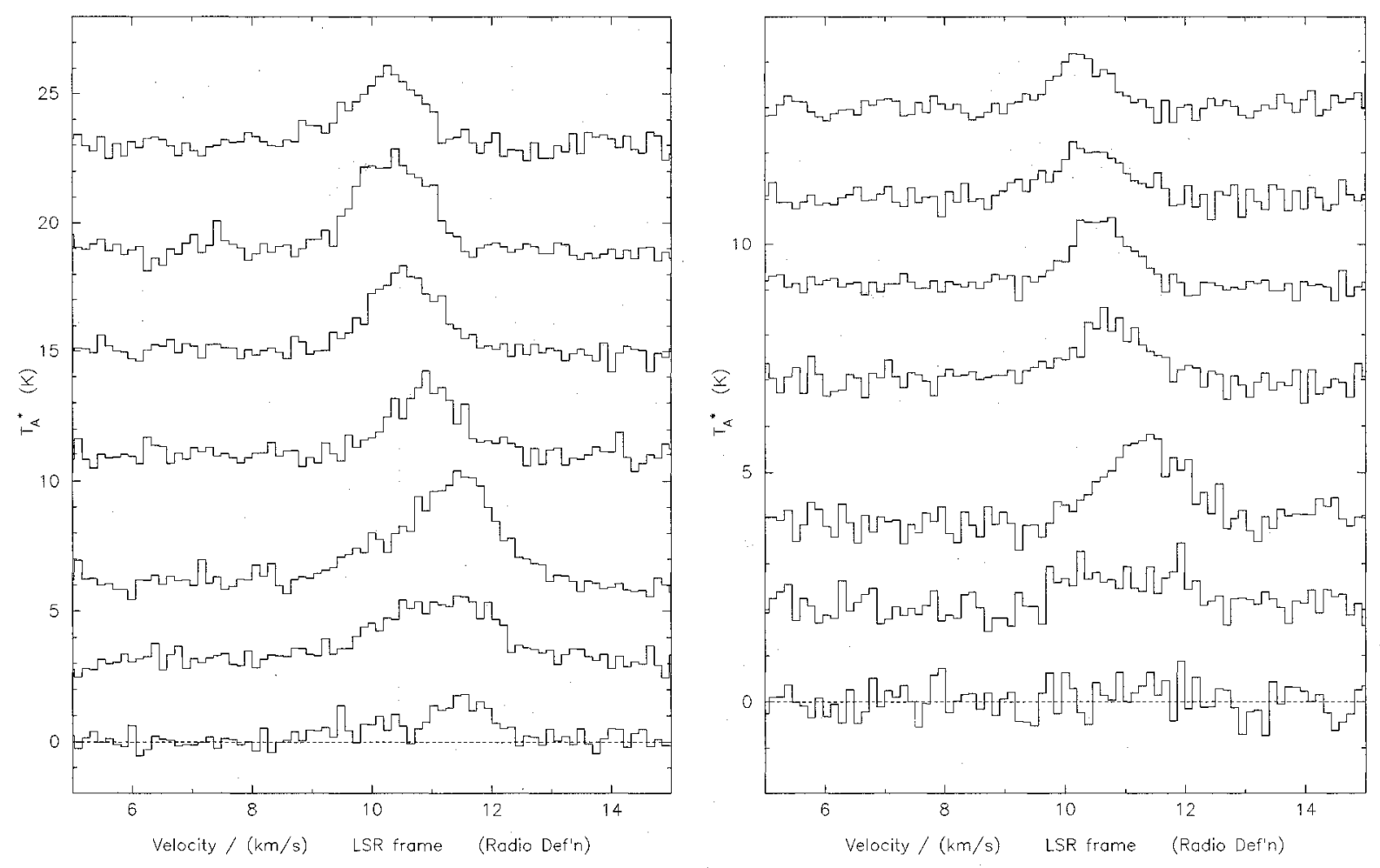

Figure 2.10: Spectra of $\mathrm{H}_{2} \mathrm{CO} 5_{05} \rightarrow 4_{04}$ line (left) and the $5_{23} \rightarrow 4_{22}$ line (right) at FIR core positions: FIRs 1-7 from top to bottom. Note the difference in the y-axis scales between the two plots. 
Table 2.4: Integrated Intensities at FIR Core Positions for $\mathrm{H}_{2} \mathrm{CO}$ Transitions

\begin{tabular}{|c|c|c|c|c|c|}
\hline Source & $\begin{array}{c}\text { Offset } \\
{\left[{ }^{\prime \prime}\right]}\end{array}$ & $\begin{array}{c}I\left(3_{03} \rightarrow 2_{02}\right)^{a} \\
{\left[\mathrm{~K} \mathrm{~km} \mathrm{~s}^{-1}\right]}\end{array}$ & $\begin{array}{c}\left(3_{22} \rightarrow 2_{21}\right)^{a} \\
{\left[\mathrm{~K} \mathrm{~km} \mathrm{~s}^{-1}\right]}\end{array}$ & $\begin{array}{c}I\left(5_{05} \rightarrow 4_{04}\right)^{a} \\
{\left[\mathrm{~K} \mathrm{~km} \mathrm{~s}^{-1}\right]}\end{array}$ & $\begin{array}{c}I\left(5_{23} \rightarrow 4_{22}\right)^{a} \\
{\left[\mathrm{~K} \mathrm{~km} \mathrm{~s}^{-1}\right]}\end{array}$ \\
\hline FIR 1 & $(-45,0)$ & $1.62 \pm 0.10$ & $0.76 \pm 0.09$ & & \\
\hline FIR 1 & $(-30,0)$ & $3.69 \pm 0.11$ & $1.22 \pm 0.09$ & $1.04 \pm 0.10$ & \\
\hline FIR 1 & $(-15,0)$ & $6.76 \pm 0.10$ & $2.12 \pm 0.09$ & $2.57 \pm 0.12$ & $0.66 \pm 0.08$ \\
\hline FIR 1 & $(0,0)$ & $6.78 \pm 0.10$ & $1.99 \pm 0.10$ & $5.00 \pm 0.16$ & $1.22 \pm 0.08$ \\
\hline FIR 1 & $(15,0)$ & $4.00 \pm 0.10$ & $1.00 \pm 0.09$ & $2.60 \pm 0.14$ & \\
\hline FIR 1 & $(30,0)$ & $1.26 \pm 0.09$ & $0.52 \pm 0.08$ & $1.67 \pm 0.11$ & $0.51 \pm 0.06$ \\
\hline FIR 1 & $(45,0)$ & $0.67 \pm 0.09$ & $0.26 \pm 0.08$ & $\ldots$ & $\ldots$ \\
\hline FIR 2 & $(-45,0)$ & $2.10 \pm 0.08$ & $0.34 \pm 0.07$ & $\ldots$ & $\ldots$ \\
\hline FIR 2 & $(-30,0)$ & $2.96 \pm 0.08$ & $0.86 \pm 0.08$ & $0.07 \pm 0.09$ & $0.25 \pm 0.06$ \\
\hline FIR 2 & $(-15,0)$ & $6.19 \pm 0.10$ & $1.60 \pm 0.08$ & $1.31 \pm 0.11$ & $0.18 \pm 0.07$ \\
\hline FIR 2 & $(0,0)$ & $8.86 \pm 0.08$ & $2.49 \pm 0.09$ & $5.54 \pm 0.14$ & $2.22 \pm 0.08$ \\
\hline FIR 2 & $(15,0)$ & $7.16 \pm 0.09$ & $2.02 \pm 0.08$ & $2.81 \pm 0.12$ & $1.38 \pm 0.09$ \\
\hline FIR 2 & $(30,0)$ & $4.27 \pm 0.09$ & $0.83 \pm 0.08$ & $1.62 \pm 0.11$ & $0.24 \pm 0.08$ \\
\hline FIR 2 & $(45,0)$ & $2.08 \pm 0.09$ & $0.16 \pm 0.09$ & $\ldots$ & $\ldots$ \\
\hline FIR 3 & $(-45,0)$ & $3.40 \pm 0.11$ & $0.49 \pm 0.08$ & $\ldots$ & $\ldots$ \\
\hline FIR 3 & $(-30,0)$ & $4.76 \pm 0.10$ & $1.03 \pm 0.09$ & $0.41 \pm 0.11$ & $0.48 \pm 0.05$ \\
\hline FIR 3 & $(-15,0)$ & $6.94 \pm 0.10$ & $1.71 \pm 0.11$ & $1.59 \pm 0.11$ & $1.03 \pm 0.07$ \\
\hline FIR 3 & $(0,0)$ & $7.65 \pm 0.11$ & $2.01 \pm 0.10$ & $5.89 \pm 0.14$ & $2.38 \pm 0.08$ \\
\hline FIR 3 & $(15,0)$ & $4.80 \pm 0.11$ & $1.16 \pm 0.10$ & $2.94 \pm 0.13$ & $1.14 \pm 0.08$ \\
\hline FIR 3 & $(30,0)$ & $2.17 \pm 0.10$ & $0.20 \pm 0.08$ & $0.26 \pm 0.10$ & $0.57 \pm 0.07$ \\
\hline FIR 3 & $(45,0)$ & $1.45 \pm 0.09$ & $0.01 \pm 0.08$ & $\cdots$ & \\
\hline FIR 4 & $(-45,0)$ & $1.56 \pm 0.11$ & $0.55 \pm 0.09$ & ... & .. \\
\hline FIR 4 & $(-30,0)$ & $2.77 \pm 0.10$ & $0.53 \pm 0.08$ & $0.02 \pm 0.10$ & $0.50 \pm 0.05$ \\
\hline FIR 4 & $(-15,0)$ & $5.32 \pm 0.10$ & $1.27 \pm 0.08$ & $1.04 \pm 0.12$ & $0.35 \pm 0.06$ \\
\hline FIR 4 & $(0,0)$ & $7.22 \pm 0.12$ & $1.68 \pm 0.09$ & $4.13 \pm 0.13$ & $3.28 \pm 0.12$ \\
\hline FIR 4 & $(15,0)$ & $5.76 \pm 0.10$ & $1.46 \pm 0.09$ & $1.98 \pm 0.13$ & $0.65 \pm 0.11$ \\
\hline FIR 4 & $(30,0)$ & $2.85 \pm 0.09$ & $0.57 \pm 0.08$ & $0.59 \pm 0.09$ & $0.28 \pm 0.06$ \\
\hline FIR 4 & $(45,0)$ & $1.15 \pm 0.10$ & $0.07 \pm 0.09$ & $\ldots$ & ... \\
\hline FIR 5 & $(-45,0)$ & $2.62 \pm 0.11$ & $0.70 \pm 0.09$ & $\ldots$ & $\ldots$ \\
\hline FIR 5 & $(-30,0)$ & $4.32 \pm 0.13$ & $1.14 \pm 0.09$ & $2.71 \pm 0.15$ & $0.81 \pm 0.15$ \\
\hline FIR 5 & $(-15,0)$ & $7.78 \pm 0.12$ & $2.61 \pm 0.10$ & $6.78 \pm 0.18$ & $1.64 \pm 0.17$ \\
\hline FIR 5 & $(0,0)$ & $9.00 \pm 0.12$ & $2.88 \pm 0.10$ & $6.65 \pm 0.18$ & $3.05 \pm 0.17$ \\
\hline FIR 5 & $(15,0)$ & $5.73 \pm 0.11$ & $1.70 \pm 0.10$ & $2.12 \pm 0.16$ & $1.65 \pm 0.11$ \\
\hline FIR 5 & $(30,0)$ & $2.99 \pm 0.11$ & $0.64 \pm 0.09$ & $1.76 \pm 0.13$ & $1.37 \pm 0.08$ \\
\hline FIR 5 & $(45,0)$ & $1.55 \pm 0.10$ & $0.37 \pm 0.08$ & $\ldots$ & $\ldots$ \\
\hline FIR 6 & $(-45,0)$ & $2.15 \pm 0.11$ & $0.62 \pm 0.10$ & .. & $\cdots$ \\
\hline FIR 6 & $(-30,0)$ & $4.01 \pm 0.12$ & $1.11 \pm 0.11$ & $\ldots$ & $\ldots$ \\
\hline FIR 6 & $(-15,0)$ & $7.28 \pm 0.12$ & $2,00 \pm 0.13$ & $1.94 \pm 0.16$ & $0.34 \pm 0.10$ \\
\hline FIR 6 & $(0,0)$ & $9.56 \pm 0.11$ & $2.40 \pm 0.12$ & $6.46 \pm 0.14$ & $2.57 \pm 0.15$ \\
\hline FIR 6 & $(15,0)$ & $6.63 \pm 0.11$ & $1.50 \pm 0.11$ & $4.35 \pm 0.17$ & $0.59 \pm 0.07$ \\
\hline FIR 6 & $(30,0)$ & $4.09 \pm 0.11$ & $1.25 \pm 0.11$ & $\ldots$ & $\cdots$ \\
\hline FIR 6 & $(45,0)$ & $2.90 \pm 0.11$ & $0.71 \pm 0.12$ & $\ldots$ & $\cdots$ \\
\hline FIR 7 & $(-45,0)$ & $1.64 \pm 0.10$ & $0.65 \pm 0.10$ & $\ldots$ & $\cdots$ \\
\hline FIR 7 & $(-30,0)$ & $3.06 \pm 0.11$ & $0.82 \pm 0.09$ & $\ldots$ & $\ldots$ \\
\hline FIR 7 & $(-15,0)$ & $5.74 \pm 0.11$ & $1.20 \pm 0.12$ & $1.26 \pm 0.12$ & $0.82 \pm 0.08$ \\
\hline FIR 7 & $(0,0)$ & $6.98 \pm 0.12$ & $1.39 \pm 0.10$ & $2.74 \pm 0.13$ & $0.08 \pm 0.11$ \\
\hline FIR 7 & $(15,0)$ & $4.48 \pm 0.10$ & $0.78 \pm 0.09$ & $1.43 \pm 0.14$ & $0.23 \pm 0.12$ \\
\hline FIR 7 & $(30,0)$ & $3.03 \pm 0.11$ & $0.90 \pm 0.09$ & $\ldots$ & $\cdots$ \\
\hline FIR 7 & $(45,0)$ & $1.97 \pm 0.10$ & $0.89 \pm 0.11$ & $\ldots$ & $\cdots$ \\
\hline
\end{tabular}

${ }^{a}$ All intensities integrated over velocity range $5-15 \mathrm{~km} \mathrm{~s}^{-1}$. 


\section{AnAlysis}

\subsection{KINETIC TEMPERATURE AND DENSITY}

The $\mathrm{H}_{2} \mathrm{CO}$ line intensity ratios $3_{03} \rightarrow 2_{02} / 3_{22} \rightarrow 2_{21}$ and $5_{05} \rightarrow 4_{04} / 5_{23} \rightarrow 4_{22}$ are useful in providing two independent measurements of the kinetic temperature of the gas. Mangum and Wootten (1993) demonstrated how the kinetic temperature from $\mathrm{H}_{2} \mathrm{CO}$ transitions may be calculated assuming that the gas is optically thin, and adopting the LTE model. Using the measurements of the integrated intensities obtained from the antenna temperatures, the following expression from Mangum and Wootten (1993) can be used to derive the kinetic temperature,

$$
T_{k i n}=\left(E_{u}^{2}-E_{u}^{1}\right)\left[\ln \left(\frac{S_{2}}{S_{1}} \frac{I_{1}}{I_{2}}\right)\right]^{-1}
$$

where $E_{u}$ is the energy of the upper level of the transition, $S$ is the line strength, and the subscripts and superscripts of 1 and 2 correspond to the $3_{03} \rightarrow 2_{02}$ and $3_{22} \rightarrow 2_{21}$ line respectively. The values used for the upper energies and the line strengths of the two transitions are given in Table 2.1.

For a reasonable evaluation of uncertainties in temperature, the lower and upper limits are calculated for the temperatures derived for the FIR core cuts. First, the averaged spectrum is observed to determine the velocity linewidth $\Delta V_{\text {line }}$ (in $\mathrm{km} \mathrm{s}^{-1}$ ). The velocity channel width $\Delta V_{c h}$ (in $\mathrm{km} \mathrm{s}^{-1}$ ) is found from $c\left(\Delta \nu / \nu_{\text {rest }}\right)$ where $c$ is the speed of light, $\Delta \nu$ is the channel width in units of frequency $(\mathrm{Hz})$ and $\nu_{\text {rest }}$ is the rest frequency of the transition in units of frequency $(\mathrm{Hz})$. The line width is then converted to units of channels by $\Delta \nu_{\text {line }} / \Delta \nu_{c h}$. The uncertainty in intensity $\Delta I$ is then found from,

$$
\Delta I=\frac{\sigma \Delta \nu_{\text {line }}}{\sqrt{n}}
$$

where $\sigma$ is the standard deviation obtained directly from SPECX for a specified velocity range. The total 
intensity ratio uncertainty $\sigma_{t o t}$ is then obtained from the following expression (Bevington, 1969):

$$
\sigma_{t o t}=\frac{I_{1}}{I_{2}} \sqrt{\left(\frac{\Delta I_{1}}{I_{1}}\right)^{2}+\left(\frac{\Delta I_{2}}{I_{2}}\right)^{2}}
$$

The upper and lower limits in temperature are derived by using the upper and lower limits of the intensity ratio:

$$
\begin{aligned}
& T_{k i n}^{u p p e r}=\left(E_{u}^{2}-E_{u}^{1}\right)\left\{\ln \left[\frac{S_{2}}{S_{1}}\left(\frac{I_{1}}{I_{2}}+\sigma_{t o t}\right)\right]\right\}^{-1} \\
& T_{k i n}^{\text {lower }}=\left(E_{u}^{2}-E_{u}^{1}\right)\left\{\ln \left[\frac{S_{2}}{S_{1}}\left(\frac{I_{1}}{I_{2}}-\sigma_{t o t}\right)\right]\right\}^{-1} .
\end{aligned}
$$

And the final temperatures with the corresponding upper and lower limits are expressed as follows:

$$
\left[T_{k i n}\right]_{\left(T_{k i n}^{\text {lower }}-T_{k i n}\right)}^{\left(T_{k i n}^{u p p e r}-T_{k i n}\right)}
$$

For the $5_{05} \rightarrow 4_{04} / 5_{23} \rightarrow 4_{22}$ transition ratio, an additional set of uncertainties are taken into account due to the calibration of two separate observations, whereas it is unnecessary for the $3_{03} \rightarrow 2_{02} / 3_{22} \rightarrow 2_{21}$ ratio, since the two lines were observed within a single beam. The calibration uncertainty of $15 \%$ is adopted, although up to $30 \%$ can be expected for $370 \mathrm{GHz}$ (Schulz et, al., 1991), for each frequency. Combining these uncertainties in the form of ratios results in a higher set of uncertainties compared with the uncertainties solely from spectral noise. The temperatures derived for various offsets at each FIR core position are given in Table 3.1. Note the large uncertainties for the $\mathrm{H}_{2} \mathrm{CO} 5_{05} \rightarrow 4_{04} / 5_{23} \rightarrow 4_{22}$ derived temperatures. Hence, the temperatures from the $3_{03} \rightarrow 2_{02} / 3_{22} \rightarrow 2_{21}$ ratio are more reliable, and these are mostly adopted for subsequent calculations.

The kinetic temperatures above are derived assuming the LTE model and optically thin gas. Another commonly used approximation is the large velocity gradient (LVG) model. Since the LTE model and LVG model use different approximations and assumptions, it is a useful check to see whether the temperatures derived by the two methods are in agreement. The LVG $H_{2}$ number densities and the observed $3_{03} \rightarrow$ 
Table 3.1: Kinetic Temperatures at FIR Core Positions

\begin{tabular}{l|ccccc}
\hline \hline Source & $\begin{array}{c}\text { Offset } \\
{["]}\end{array}$ & $\begin{array}{c}I\left(33_{33} \rightarrow 2_{02}\right) \\
I\left(3_{22} \rightarrow 2_{21}\right)\end{array}$ & $\begin{array}{c}T_{k i n}\left(\frac{3_{03} \rightarrow 2_{02}}{3_{22} \rightarrow 2_{21}}\right) \\
{[\mathrm{K}]}\end{array}$ & $\frac{I\left(5_{00} \rightarrow 4_{04}\right)}{I\left(5_{23} \rightarrow 44_{22}\right)}$ & $\begin{array}{c}T_{k i n}\left(\frac{5_{05} \rightarrow 4_{01}}{5_{23} \rightarrow 4_{22}}\right)^{a} \\
{[\mathrm{~K}]}\end{array}$ \\
\hline FIR 1 & $(-15,0)$ & $3.19 \pm 0.15$ & $82_{-8}^{+9}$ & $1.65 \pm 0.35$ & $145_{-59}^{+389}$ \\
FIR 1 & $(0,0)$ & $3.41 \pm 0.18$ & $74_{-7}^{+8}$ & $3.31 \pm 0.70$ & $46_{-8}^{+14}$ \\
FIR 1 & $(15,0)$ & $4.00 \pm 0.36$ & $59_{-8}^{+9}$ & $\ldots$ & $\ldots$ \\
FIR 2 & $(-15,0)$ & $3.87 \pm 0.21$ & $61_{-5}^{+6}$ & $2.15 \pm 0.46$ & $80_{-22}^{+54}$ \\
FIR 2 & $(0,0)$ & $3.56 \pm 0.13$ & $69_{-4}^{+5}$ & $2.72 \pm 0.58$ & $57_{-12}^{+23}$ \\
FIR 2 & $(15,0)$ & $3.55 \pm 0.15$ & $69_{-5}^{+6}$ & $2.55 \pm 0.54$ & $62_{-14}^{+28}$ \\
FIR 3 & $(-15,0)$ & $4.06 \pm 0.26$ & $58_{-5}^{+6}$ & $7.39 \pm 1.57$ & $26_{-3}^{+4}$ \\
FIR 3 & $(0,0)$ & $3.81 \pm 0.20$ & $63_{-5}^{+6}$ & $1.79 \pm 0.38$ & $116_{-41}^{+161}$ \\
FIR 3 & $(15,0)$ & $4.14 \pm 0.36$ & $57_{-7}^{+8}$ & $7.03 \pm 1.49$ & $27_{-3}^{+4}$ \\
FIR 4 & $(-15,0)$ & $4.19 \pm 0.27$ & $56_{-5}^{+6}$ & $\ldots$ & $\ldots$ \\
FIR 4 & $(0,0)$ & $4.30 \pm 0.25$ & $54_{-4}^{+5}$ & $2.09 \pm 0.44$ & $84_{-24}^{+62}$ \\
FIR 4 & $(15,0)$ & $3.95 \pm 0.25$ & $60_{-6}^{+6}$ & $3.60 \pm 0.76$ & $43_{-7}^{+12}$ \\
FIR 5 & $(-15,0)$ & $2.98 \pm 0.12$ & $93_{-9}^{+11}$ & $1.79 \pm 1.00$ & $34_{-5}^{+7}$ \\
FIR 5 & $(0,0)$ & $3.13 \pm 0.12$ & $85_{-7}^{+8}$ & $3.72 \pm 0.79$ & $42_{-7}^{+11}$ \\
FIR 5 & $(15,0)$ & $3.37 \pm 0.22$ & $75_{-9}^{+11}$ & $1.79 \pm 0.38$ & $117_{-42}^{+166}$ \\
FIR 6 & $(-15,0)$ & $3.64 \pm 0.24$ & $67_{-7}^{+8}$ & $2.27 \pm 0.48$ & $73_{-19}^{+43}$ \\
FIR 6 & $(0,0)$ & $3.98 \pm 0.20$ & $59_{-4}^{+5}$ & $3.11 \pm 0.66$ & $49_{-9}^{+16}$ \\
FIR 6 & $(15,0)$ & $4.42 \pm 0.33$ & $52_{-5}^{+6}$ & $10.00 \pm 2.12$ & $22_{-2}^{+3}$ \\
FIR 7 & $(-15,0)$ & $4.78 \pm 0.48$ & $48_{-5}^{+6}$ & $3.41 \pm 0.72$ & $45_{-8}^{+13}$ \\
FIR 7 & $(0,0)$ & $5.02 \pm 0.39$ & $46_{-4}^{+4}$ & $8.62 \pm 1.83$ & $24_{-2}^{+3}$ \\
FIR 7 & $(15,0)$ & $5.74 \pm 0.68$ & $41_{-5}^{+5}$ & $4.19 \pm 0.89$ & $38_{-6}^{+9}$ \\
\hline
\end{tabular}

${ }^{a}$ Uncertainties from $5_{05} \rightarrow 4_{04} / 5_{23} \rightarrow 4_{22}$ ratios arise from calibration uncertainties and systematic error. 
Table 3.2: LTE and LVG Derived Temperature Comparison

\begin{tabular}{|c|c|c|c|c|c|}
\hline \multirow[t]{3}{*}{ Source } & \multirow{3}{*}{$\begin{array}{c}\text { Offset } \\
{\left[{ }^{\prime \prime}\right]}\end{array}$} & \multicolumn{2}{|c|}{$T_{k i n}\left(\frac{303 \rightarrow 202}{30}\right)$} & \multicolumn{2}{|c|}{$\bar{T}_{k \text { kin }}\left(\frac{5_{05} \rightarrow 4_{04}}{5}\right)$} \\
\hline & & LTE & $\mathrm{LVG}^{a}$ & LTE & $\mathrm{LVG}^{a}$ \\
\hline & & {$[\mathrm{K}]$} & {$[\mathrm{K}]$} & {$[\mathrm{K}]$} & [K] \\
\hline FIR 1 & $(-15,0)$ & $82_{-8}^{+9}$ & $90 \pm 10$ & $145_{-47}^{+131}$ & $120 \pm 10$ \\
\hline FIR 1 & $(0,0)$ & $74_{-7}^{+8}$ & $80 \pm 10$ & $46_{-4}^{+4}$ & $50 \pm 10$ \\
\hline FIR 1 & $(15,0)$ & $59_{-8}^{+9}$ & $60 \pm 10$ & & ‥ \\
\hline FIR 2 & $(-15,0)$ & $61_{-5}^{+6}$ & $50 \pm 10$ & $80_{-27}^{+108}$ & $60 \pm 10$ \\
\hline FIR 2 & $(0,0)$ & $69_{-4}^{+5}$ & $70 \pm 10$ & $57_{-4}^{+5}$ & $60 \pm 10$ \\
\hline FIR 2 & $(15,0)$ & $69_{-5}^{+6}$ & $70 \pm 10$ & $62_{-7}^{+\frac{9}{9}}$ & $60 \pm 10$ \\
\hline FIR 3 & $(-15,0)$ & $58_{-5}^{+6}$ & $50 \pm 10$ & $26_{-5}^{+11}$ & . \\
\hline FIR 3 & $(0,0)$ & $63_{-5}^{+6}$ & $70 \pm 10$ & $116_{-16}^{+19}$ & $100 \pm 10$ \\
\hline FIR 3 & $(15,0)$ & $57_{-7}^{+8}$ & $60 \pm 10$ & $27_{-2}^{+2}$ & ‥ \\
\hline FIR 4 & $(-15,0)$ & $56_{-5}^{+6}$ & $60 \pm 10$ & & \\
\hline FIR 4 & $(0,0)$ & $54_{-4}^{+5}$ & $60 \pm 10$ & $84_{-12}^{+14}$ & $70 \pm 10$ \\
\hline FIR 4 & $(15,0)$ & $60_{-6}^{+6}$ & $50 \pm 10$ & $43_{-5}^{+6}$ & $40 \pm 10$ \\
\hline FIR 5 & $(-15,0)$ & $93_{-9}^{+11}$ & $100 \pm 10$ & $34_{-4}^{+6}$ & $40 \pm 10$ \\
\hline FIR 5 & $(0,0)$ & $85_{-7}^{+8}$ & $100 \pm 10$ & $42_{-3}^{+3}$ & $40 \pm 10$ \\
\hline FIR 5 & $(15,0)$ & $75_{-9}^{+11}$ & $80 \pm 10$ & $117_{-21}^{+30}$ & $100 \pm 10$ \\
\hline FIR 6 & $(-15,0)$ & $67_{-7}^{+8}$ & $70 \pm 10$ & $73_{-18}^{+37}$ & $60 \pm 10$ \\
\hline FIR 6 & $(0,0)$ & $59_{-4}^{+5}$ & $60 \pm 10$ & $49_{-4}^{+5}$ & $50 \pm 10$ \\
\hline FIR 6 & $(15,0)$ & $52_{-5}^{+6}$ & $50 \pm 10$ & $22_{-2}^{+2}$ & . \\
\hline FIR 7 & $(-15,0)$ & $48_{-5}^{+6}$ & $50 \pm 10$ & $45_{-9}^{+18}$ & $50 \pm 10$ \\
\hline FIR 7 & $(0,0)$ & $46_{-4}^{+4}$ & $40 \pm 10$ & $24_{-3}^{+6}$ & \\
\hline FIR 7 & $(15,0)$ & $41_{-5}^{+5}$ & $40 \pm 10$ & $38_{-8}^{+17}$ & $30 \pm 10$ \\
\hline
\end{tabular}

${ }^{a}$ LVG temperatures estimated from LVG models of van Dishoeck et al. (1993).

$2_{02} / 3_{22} \rightarrow 2_{21}$ and $5_{05} \rightarrow 4_{04} / 5_{23} \rightarrow 4_{22}$ line ratios can be used to find the LVG temperature estimates from van Dishoeck et al. (1993). In contrast to the $3_{03} \rightarrow 2_{02} / 5_{05} \rightarrow 4_{04}$ ratio, these two line ratios are sensitive to temperature rather than density. Table 3.2 shows the estimated LVG temperatures alongside the LTE temperatures for comparison. These temperatures were estimated from a plot of select line ratios, so the uncertainties arise from lack or resolution in the given plots. Hence, the chosen uncertainties for all LVG temperature estimates are the same. The two sets of temperatures give good agreement within their uncertainties, and is discussed further in Section 4.

Intensity ratios of $3_{03} \rightarrow 2_{02} / 5_{05} \rightarrow 4_{04}$, together with gas kinetic temperatures, offer number density constraints in the LVG approximation. van Dishoeck et al. (1993) have used LVG models to calculate the $3_{03} \rightarrow 2_{02} / 5_{05} \rightarrow 4_{04}$ intensity ratio for a range of $H_{2}$ densities and gas kinetic temperatures. Their results are used here to obtain $\mathrm{H}_{2}$ densities from formaldehyde $3_{03} \rightarrow 2_{02} / 5_{05} \rightarrow 4_{04}$ intensity ratio. Table 3.3 shows the $\mathrm{H}_{2} \mathrm{CO}$ number density estimates for various offsets of each FIR core. As with the LVG 
Table 3.3: $H_{2}$ Number Densities at FIR Core Positions

\begin{tabular}{c|ccc}
\hline \hline Source & $\begin{array}{c}\text { Offset } \\
{\left[{ }^{\prime}\right]}\end{array}$ & $\begin{array}{c}\frac{T\left(3_{03} \rightarrow 2_{02}\right)}{I\left(5_{05} \rightarrow 4_{04}\right)} \\
\text { FIR 1 }\end{array}$ & $\begin{array}{c}n(15,0) \\
{\left[\mathrm{cm}^{-3}\right]}\end{array}$ \\
FIR 1 & $(0,0)$ & $1.31 \pm 0.12$ & $1 \times 10^{6} \pm 0.5 \times 10^{6}$ \\
FIR 1 & $(-15,0)$ & $1.61 \pm 0.17$ & $2 \times 10^{6} \pm 0.5 \times 10^{6}$ \\
FIR 2 & $(15,0)$ & $4.33 \pm 0.41$ & $6 \times 10^{6} \pm 0.5 \times 10^{6}$ \\
FIR 2 & $(0,0)$ & $1.79 \pm 0.07$ & $2 \times 10^{6} \pm 0.5 \times 10^{6}$ \\
FIR 2 & $(-15,0)$ & $2.74 \pm 0.16$ & $1 \times 10^{6} \pm 0.5 \times 10^{6}$ \\
FIR 3 & $(15,0)$ & $4.49 \pm 0.37$ & $5 \times 10^{5} \pm 0.5 \times 10^{5}$ \\
FIR 3 & $(0,0)$ & $1.67 \pm 0.06$ & $2 \times 10^{6} \pm 0.5 \times 10^{6}$ \\
FIR 3 & $(-15,0)$ & $2.23 \pm 0.13$ & $2 \times 10^{6} \pm 0.5 \times 10^{6}$ \\
FIR 4 & $(15,0)$ & $2.21 \pm 0.18$ & $2 \times 10^{6} \pm 0.5 \times 10^{6}$ \\
FIR 4 & $(0,0)$ & $2.05 \pm 0.11$ & $2 \times 10^{6} \pm 0.5 \times 10^{6}$ \\
FIR 4 & $(-15,0)$ & $4.01 \pm 0.36$ & $6 \times 10^{5} \pm 0.5 \times 10^{5}$ \\
FIR 5 & $(15,0)$ & $1.40 \pm 0.07$ & $2 \times 10^{6} \pm 0.5 \times 10^{6}$ \\
FIR 5 & $(0,0)$ & $1.12 \pm 0.04$ & $2 \times 10^{6} \pm 0.5 \times 10^{6}$ \\
FIR 5 & $(-15,0)$ & $1.37 \pm 0.08$ & $2 \times 10^{6} \pm 0.5 \times 10^{6}$ \\
FIR 6 & $(15,0)$ & $3.74 \pm 0.29$ & $1 \times 10^{6} \pm 0.5 \times 10^{6}$ \\
FIR 6 & $(0,0)$ & $1.85 \pm 0.06$ & $2 \times 10^{6} \pm 0.5 \times 10^{6}$ \\
FIR 6 & $(-15,0)$ & $1.79 \pm 0.10$ & $2 \times 10^{6} \pm 0.5 \times 10^{6}$ \\
FIR 7 & $(15,0)$ & $3.48 \pm 0.30$ & $2 \times 10^{6} \pm 0.5 \times 10^{6}$ \\
FIR 7 & $(0,0)$ & $3.40 \pm 0.23$ & $2 \times 10^{6} \pm 0.5 \times 10^{6}$ \\
FIR 7 & $(-15,0)$ & $6.34 \pm 0.85$ & $5 \times 10^{5} \pm 0.5 \times 10^{5}$ \\
\hline
\end{tabular}

${ }^{a}$ Values estimated from LVG models of van Dishoeck et al. (1993) using $\mathrm{H}_{2} \mathrm{CO}$ intensity ratio $3_{03} \rightarrow 2_{02} / 5_{05} \rightarrow 4_{04}$.

temperature estimates, the LVG model plots are offered for only a select number of intensity ratio values. The uncertainties are therefore reflective of the limited resolution of the plots.

In the optically thin limit, the column density is given by,

$$
N_{t o t}=\frac{8 \pi k \nu_{u l}^{2} Q\left(T_{r o t}\right)}{h c^{3} A_{u l} g_{u}} e^{\frac{E_{u}}{k T_{r o t}}} \int T_{m b} d \nu
$$

where $\nu_{u l}$ is the transition frequency, $A_{u l}$ is the Einstein coefficient for spontaneous transition, $g_{u}$ is the statistical weight of the upper state, $Q\left(T_{\text {rot }}\right)$ is the partition function as a function of rotational temperature $\left(T_{\text {rot }}\right), E_{u}$ is the energy of the upper state, $k$ is the Boltzmann constant, $h$ is the Planck constant, $c$ is the speed of light, and $\int T_{m b} d \nu$ is the main beam temperature integrated over the velocity linewidth $d \nu$ in units 
of $\mathrm{K} \mathrm{km} \mathrm{s}^{-1}$. For $\mathrm{H}_{2} \mathrm{CO}$, this equation can be simplified to,

$$
\frac{N_{t o t}}{Q\left(T_{r o t}\right)} e^{-\frac{E_{u}}{k T_{r o t}}}=\frac{1.67 \times 10^{14}}{\nu \mu^{2} S} \int T_{m b} d \nu
$$

where $\mu$ is the permanent dipole moment and $S$ is the line strength. Using the expression from Blake et al. (1987), the partition function can be given by:

$$
Q\left(T_{r o t}\right)=2\left[\frac{\pi\left(k T_{\text {rot }}\right)^{3}}{h^{3} A B C}\right]^{1 / 2}
$$

The rotational constants $A, B$, and $C$ were obtained from the JPL catalog of spectroscopy ${ }^{1}$. The total $\mathrm{H}_{2} \mathrm{CO}$ column densities derived from the above equations are given in Table 3.4. If an appropriate value for the $\mathrm{H}_{2} \mathrm{CO}$ fractional abundance relative to $\mathrm{H}_{2}$ was known at specific regions of NGC 2024, the total $\mathrm{H}_{2}$ column densities may be calculated. This is not entirely reliable, however, since a constant abundance ratio for such an extensive region is unlikely, and the fractional abundance varies according to the temperature of the region. Instead of calculating the $\mathrm{H}_{2}$ column densities, the fractional abundance at the FIR core positions are derived using these $\mathrm{H}_{2} \mathrm{CO}$ column densities and the $\mathrm{H}_{2}$ column densities from previous data (Johnstone et al., 2006). These calculations are presented in section 3.5 of this chapter, and are discussed further in chapter 4 .

\subsection{MASS}

Masses for clumps or cores may be found by various methods, using known properties such as densities and sizes. One approach is to make use of emission from dust, and to assume a dust temperature. A recent study of NGC 2024 using SCUBA $850 \mu \mathrm{m}$ data by Johnstone et al. (2006) in combination with the gas kinetic temperatures obtained from formaldehyde transitions allows for the derivation of core masses. From Johnstone et al. (2006),

$$
\frac{M_{\text {clump }}}{M_{\odot}}=0.59 S_{850}\left[\exp \left(\frac{17 K}{T_{d}}\right)-1\right]\left(\frac{\kappa_{850}}{0.02 c m^{2} g^{-1}}\right)^{-1}\left(\frac{d}{400 p c}\right)^{2}
$$

\footnotetext{
${ }^{1} \mathrm{JPL}$ molecular spectroscopy site http://spec.jpl.nasa.gov/
} 
Table 3.4: $\mathrm{H}_{2} \mathrm{CO}$ Column Densities at FIR Core Positions

\begin{tabular}{l|ccc}
\hline \hline Source & $\begin{array}{c}\text { Offset } \\
{\left[{ }^{\prime \prime}\right]}\end{array}$ & $\begin{array}{c}N\left(\mathrm{H}_{2} \mathrm{CO}\right)_{3_{03} \rightarrow 202} \\
{\left[\mathrm{~cm}^{-2}\right]}\end{array}$ & $\begin{array}{c}N\left(\mathrm{H}_{2} \mathrm{CO}\right)_{3_{22} \rightarrow 2_{21}} \\
{\left[\mathrm{~cm}^{-2}\right]}\end{array}$ \\
\hline FIR 1 & $(-15,0)$ & $2.3 \times 10^{14} \pm 2.6 \times 10^{13}$ & $7.2 \times 10^{14} \pm 4.6 \times 10^{13}$ \\
FIR 1 & $(0,0)$ & $2.0 \times 10^{14} \pm 9.1 \times 10^{13}$ & $6.7 \times 10^{14} \pm 3.7 \times 10^{13}$ \\
FIR 1 & $(15,0)$ & $8.9 \times 10^{13} \pm 1.9 \times 10^{13}$ & $3.6 \times 10^{14} \pm 1.9 \times 10^{13}$ \\
FIR 2 & $(-15,0)$ & $1.5 \times 10^{14} \pm 1.0 \times 10^{14}$ & $5.6 \times 10^{14} \pm 1.8 \times 10^{13}$ \\
FIR 2 & $(0,0)$ & $2.4 \times 10^{14} \pm 3.0 \times 10^{13}$ & $8.5 \times 10^{14} \pm 2.7 \times 10^{13}$ \\
FIR 2 & $(15,0)$ & $1.9 \times 10^{14} \pm 1.1 \times 10^{14}$ & $6.9 \times 10^{14} \pm 2.5 \times 10^{13}$ \\
FIR 3 & $(-15,0)$ & $1.5 \times 10^{14} \pm 4.4 \times 10^{13}$ & $6.2 \times 10^{14} \pm 2.0 \times 10^{13}$ \\
FIR 3 & $(0,0)$ & $1.8 \times 10^{14} \pm 6.8 \times 10^{13}$ & $7.0 \times 10^{14} \pm 2.4 \times 10^{13}$ \\
FIR 3 & $(15,0)$ & $1.0 \times 10^{14} \pm 7.0 \times 10^{13}$ & $4.2 \times 10^{14} \pm 1.8 \times 10^{13}$ \\
FIR 4 & $(-15,0)$ & $1.1 \times 10^{14} \pm 4.3 \times 10^{13}$ & $4.7 \times 10^{14} \pm 1.3 \times 10^{13}$ \\
FIR 4 & $(0,0)$ & $1.5 \times 10^{14} \pm 1.2 \times 10^{13}$ & $6.3 \times 10^{14} \pm 1.3 \times 10^{13}$ \\
FIR 4 & $(15,0)$ & $1.3 \times 10^{14} \pm 7.2 \times 10^{13}$ & $5.2 \times 10^{14} \pm 1.9 \times 10^{13}$ \\
FIR 5 & $(-15,0)$ & $3.0 \times 10^{14} \pm 3.8 \times 10^{13}$ & $9.0 \times 10^{14} \pm 6.4 \times 10^{13}$ \\
FIR 5 & $(0,0)$ & $3.1 \times 10^{14} \pm 1.2 \times 10^{14}$ & $9.8 \times 10^{14} \pm 5.2 \times 10^{13}$ \\
FIR 5 & $(15,0)$ & $1.7 \times 10^{14} \pm 9.7 \times 10^{13}$ & $5.7 \times 10^{14} \pm 4.3 \times 10^{13}$ \\
FIR 6 & $(-15,0)$ & $1.9 \times 10^{14} \pm 4.5 \times 10^{13}$ & $6.9 \times 10^{14} \pm 3.8 \times 10^{13}$ \\
FIR 6 & $(0,0)$ & $2.2 \times 10^{14} \pm 7.1 \times 10^{13}$ & $8.6 \times 10^{14} \pm 2.3 \times 10^{13}$ \\
FIR 6 & $(15,0)$ & $1.3 \times 10^{14} \pm 1.4 \times 10^{13}$ & $5.7 \times 10^{14} \pm 1.3 \times 10^{13}$ \\
FIR 7 & $(-15,0)$ & $1.0 \times 10^{14} \pm 2.5 \times 10^{13}$ & $4.9 \times 10^{14} \pm 9.3 \times 10^{12}$ \\
FIR 7 & $(0,0)$ & $1.2 \times 10^{14} \pm 4.3 \times 10^{13}$ & $6.0 \times 10^{14} \pm 3.6 \times 10^{12}$ \\
FIR 7 & $(15,0)$ & $6.7 \times 10^{13} \pm 2.9 \times 10^{13}$ & $3.9 \times 10^{14} \pm 9.4 \times 10^{12}$ \\
\hline
\end{tabular}

where $T_{d}$ is the dust temperature, $S_{850}$ is the $850 \mu \mathrm{m}$ flux, $\kappa_{850}$ is the mass absorption coefficient 0.02 $\mathrm{cm}^{2} \mathrm{~g}^{-1}$ from Johnstone et al. (2006), and $d$ is the distance to the source $415 \mathrm{pc}$. The dust temperature is assumed to be equal to the gas kinetic temperature, $T_{k i n}$. This should be valid for the very high gas densities in NGC 2024 (see Table 3.3). Johnstone et al. (2006) applied a clump finder to the $850 \mu \mathrm{m}$ map and presented the size of each clump, the total flux inside the clump, and the clump mass for a dust temperature of $20 \mathrm{~K}$. Using the $\mathrm{H}_{2} \mathrm{CO}$ derived temperatures from the ratio $3_{03} \rightarrow 2_{02} / 3_{22} \rightarrow 2_{21}$, the clump masses are rederived, and also the mass within a $15^{\prime \prime}$ beam. The results are shown in Table 3.5. The clump masses calculated here are used to evaluate the virial stability of the FIR cores in section 4.4.2.

\section{3 $\mathrm{HCO}^{+}$AND $\mathrm{H}^{13} \mathrm{CO}^{+}$}

With the anticipation of detecting possible infall at the core positions, the FIR cores were observed in $\mathrm{HCO}^{+}$ $(J=3-2)$ and $\mathrm{H}^{13} \mathrm{CO}^{+}(J=3-2)$. The expected double-peaked lines are observed for $\mathrm{HCO}^{+}$, while the $\mathrm{H}^{13} \mathrm{CO}^{+}$lines are all single peaked (as seen in Fig. 2.8), so the possibility of multiple velocity components 
Table 3.5: Masses Derived for FIRs 3, 4,5, and 6

\begin{tabular}{c|cc}
\hline \hline \multirow{3}{*}{ Source } & \multicolumn{2}{|c}{$M\left(T_{\mathrm{H}_{2} C O}\right)^{a b}$} \\
& $\begin{array}{c}\text { per 15" beam } \\
\text { clump total }\end{array}$ \\
& {$\left[\mathrm{M}_{\odot}\right.$ beam $\left.^{-1}\right]$} & {$\left[\mathrm{M}_{\odot}\right]$} \\
\hline FIR 3 & 2.0 & 15.6 \\
FIR 4 & 1.7 & 14.0 \\
FIR 5 & 2.0 & 12.0 \\
FIR 6 & 2.0 & 22.4 \\
\hline
\end{tabular}

${ }^{a}$ Used temperatures derived from $\mathrm{H}_{2} \mathrm{CO}$ line ratio $3_{03} \rightarrow 2_{02} / 3_{22} \rightarrow 2_{21}$.

${ }^{b}$ Mass absorption coefficient of $\kappa=0.02$ $\mathrm{cm}^{2} \mathrm{~g}^{-1}$ used.

${ }^{c}$ Masses derived for clump sizes given by Johnstone et al. (2006).

is ruled out. The velocities at which the $\mathrm{H}^{13} \mathrm{CO}^{+}$lines peaked are approximately at the same velocities as the self-absorption dips of the $\mathrm{HCO}^{+}$lines, further supporting this interpretation. The possibility of infalling cores, however, cannot be established, since the $\mathrm{HCO}^{+}$peaks show red-skewed profiles, rather than blue-skewed profiles. The implications of a red-skewed profile are remarked upon in chapter 4 . The $\mathrm{HCO}^{+}$ spectra were therefore all treated as self-absorption lines. Since the self-absorbed portions of the lines can affect the outcome of calculations, they were corrected for by fitting each spectrum with a Gaussian curve. The fitted Gaussian lines were used to evaluate the antenna temperature. Fig. 3.1 shows the best-fitting Gaussian curves for the $\mathrm{HCO}^{+}$spectra from each core.

\subsubsection{OPTICAL DEPTH AND EXCITATION TEMPERATURE}

The intensities from the two lines of $\mathrm{HCO}^{+} J=3-2$ and $\mathrm{H}^{13} \mathrm{CO}^{+} J=3-2$ can give a ratio which allows for the optical depth $(\tau)$ to be calculated. For any optical depth $\tau$, the radiation temperature is given by,

$$
T_{R}=\left(1-e^{-\tau}\right) \frac{h \nu / k}{e^{h \nu / k T_{c x}}-1}
$$

where the radiation temperature is related to the observed antenna temperature by $T_{R}=T_{A}^{*} / \eta_{m b}$ as described in section 3.1. Provided the two transitions have the same excitation temperature $\left(T_{e x}\right)$, and neglecting the small difference in frequency between the two transitions so $\nu\left(\mathrm{HCO}^{+}\right) \cong \nu\left(\mathrm{H}^{13} \mathrm{CO}^{+}\right)$, the 


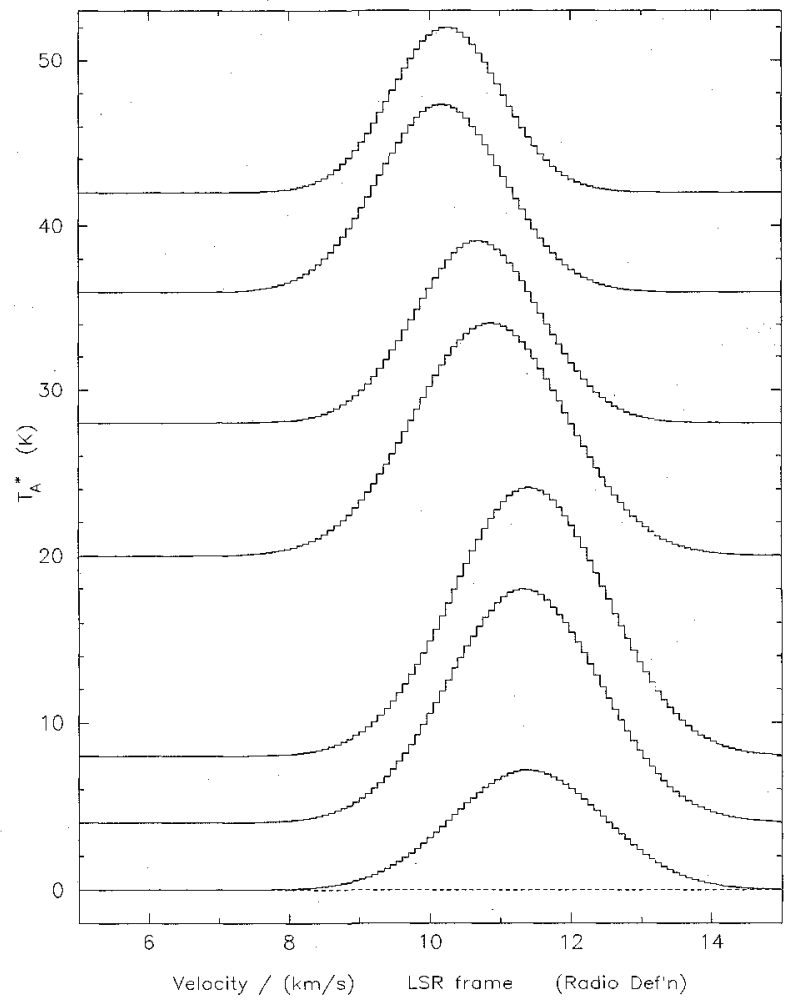

Figure 3.1: Best fitting Gaussian curves for spectra of $\mathrm{HCO}^{+}$lines at $267 \mathrm{GHz}$ to correct for self-absorption at positions of FIR 1-7 (top to bottom). 
Table 3.6: Optical Depths and Excitation Temperatures at FIR Core Positions

\begin{tabular}{c|ccccc}
\hline \hline Source & $\frac{T_{A}^{*}\left(\mathrm{HCO}^{+}\right)}{T_{A}^{*}\left(\mathrm{H}^{13} \mathrm{CO}\right)}$ & $\tau\left(H^{13} \mathrm{CO}+\right)^{a}$ & $\tau(\mathrm{HCO+})^{a}$ & $\begin{array}{c}T_{e x}\left(H^{13} \mathrm{CO}^{+}\right)^{b} \\
{[\mathrm{~K}]}\end{array}$ & $\begin{array}{c}T_{\text {ex }}\left(\mathrm{HCO}^{+}\right)^{b} \\
{[\mathrm{~K}]}\end{array}$ \\
\hline FIR 1 & $9.9 \pm 0.1$ & 0.1 & 6.4 & 34 & 34 \\
FIR 2 & $8.3 \pm 0.2$ & 0.1 & 7.7 & 42 & 43 \\
FIR 3 & $9.4 \pm 0.1$ & 0.1 & 6.7 & 42 & 42 \\
FIR 4 & $20.3 \pm 0.2$ & 0.1 & 2.9 & 64 & 64 \\
FIR 5 & $9.0 \pm 0.2$ & 0.1 & 7.0 & 70 & 70 \\
FIR 6 & $12.1 \pm 0.2$ & 0.1 & 5.2 & 61 & 61 \\
FIR 7 & $10.4 \pm 0.2$ & 0.1 & 6.1 & 34 & 34 \\
\hline
\end{tabular}

${ }^{a}$ uncertainties \pm less than $1 \%$

${ }^{b}$ uncertainties \pm less than $2 \%$

following relationship can be used to find the optical depths for both $\mathrm{HCO}^{+}$and $\mathrm{H}^{13} \mathrm{CO}^{+}$,

$$
\frac{T_{A}^{*}\left(H C O^{+}\right)}{T_{A}^{*}\left(H^{13} C O^{+}\right)} \cong \frac{1-e^{-\tau\left(H C O^{+}\right)}}{1-e^{-\tau\left(H^{13} C O^{+}\right)}}=\frac{1-e^{-A \tau\left(H^{13} C O^{+}\right)}}{1-e^{-\tau\left(H^{13} C O^{+}\right)}}
$$

where $A$ is the abundance ratio $\mathrm{HCO}^{+} / \mathrm{H}^{13} \mathrm{CO}^{+}$, and $\tau\left(\mathrm{HCO}^{+}\right)=A \tau\left(\mathrm{H}^{13} \mathrm{CO}^{+}\right)$. The excitation temperature can then be found, given the optical depth and the observed radiation temperature:

$$
T_{e x}=\left\{\frac{k}{h \nu} \ln \left[\left(1-e^{-\tau}\right) \frac{h \nu / k}{k T_{R}}+1\right]\right\}^{-1} .
$$

Using the abundance ratio of $A=60$, the optical depths were derived from the observed $\mathrm{H}^{13} \mathrm{CO}^{+}$antenna temperatures and absorption-corrected $\mathrm{HCO}^{+}$antenna temperatures for each FIR core position. The excitation temperatures were then calculated from the derived optical depths for both transitions. The final values are given in Table 3.6.

The derived excitation temperatures deviate from the $\mathrm{H}_{2} \mathrm{CO}$ derived kinetic temperatures. Although a few FIR core excitation temperatures are very similar to the kinetic temperatures from $\mathrm{H}_{2} \mathrm{CO}$ (FIRs 4 and 6), the others are as different as up to $\sim 40 \mathrm{~K}$. Comparisons between the two sets of temperatures and their explanations will be discussed in more detail in Section 4. 
Table 3.7: $\mathrm{H}^{13} \mathrm{CO}^{+}$and $\mathrm{HCO}^{+}$Column Densities at FIR Core Positions

\begin{tabular}{c|cc}
\hline \hline Source & $\begin{array}{c}N\left(\mathrm{H}^{13} \mathrm{CO}^{+}\right) \\
{\left[\mathrm{cm}^{-2}\right]}\end{array}$ & $\begin{array}{c}N\left(\mathrm{HCO}^{+}\right) \\
{\left[\mathrm{cm}^{-2}\right]}\end{array}$ \\
\hline FIR 1 & $1.5 \times 10^{12}$ & $8.8 \times 10^{13}$ \\
FIR 2 & $2.5 \times 10^{12}$ & $1.5 \times 10^{14}$ \\
FIR 3 & $2.1 \times 10^{12}$ & $1.3 \times 10^{14}$ \\
FIR 4 & $1.8 \times 10^{12}$ & $1.1 \times 10^{14}$ \\
FIR 5 & $5.1 \times 10^{12}$ & $3.0 \times 10^{14}$ \\
FIR 6 & $3.0 \times 10^{12}$ & $1.8 \times 10^{14}$ \\
FIR 7 & $1.4 \times 10^{12}$ & $8.2 \times 10^{13}$ \\
\hline
\end{tabular}

\subsubsection{COLUMN DENSITY}

Using the observed radiation temperature, the calculated optical depth, and the excitation temperature, the $\mathrm{H}^{13} \mathrm{CO}^{+}$column densities are derived from the expression given by Hogerheijde et al. (1997),

$$
N=10^{5} \frac{3 k^{2}}{4 h \pi^{3} \mu^{2} \nu^{2}} e^{\frac{h \nu J_{l}}{2 k T_{e x}}} \frac{T_{e x}+h \nu / 6 k\left(J_{l}+1\right)}{e^{h \nu / k T_{e x}}} \int T_{R}\left(\frac{\tau}{1-e^{-\tau}}\right) d V
$$

which is a simplified version of an expression from Scoville et al. (1986):

$$
N=\frac{3 k}{8 \pi^{3} B \mu^{2}} \frac{e^{h B J_{l}\left(J_{l}+1\right) / k T_{e x}}}{J_{l}+1} \frac{T_{e x}+h B / 3 k}{1-e^{-h \nu / k T_{e x}}} \int T_{R} d V
$$

The column densities for $\mathrm{HCO}^{+}$is then obtained by multiplying the column densities from $\mathrm{H}^{13} \mathrm{CO}^{+}$by the abundance ratio $A(60)$. Table 3.7 shows the column densities derived for $\mathrm{H}^{13} \mathrm{CO}^{+}$and $\mathrm{HCO}^{+}$at the FIR core positions. Fractional abundances for $\mathrm{HCO}^{+} / \mathrm{H}_{2}$ are derived from these column densities and presented in section 3.5 .

\subsection{Column DENSity From EXtinction AND $850 \mu \mathrm{M}$}

Haisch et al. (2001) presented infrared photometry of the NGC 2024 embedded star cluster. In this study, the authors surveyed the region in search of circumstellar disks around YSOs at mid-IR $(10.8 \mu \mathrm{m})$. Whereever possible, their data were used in conjunction with previous $J H K L$ photometry data to derive visual extinctions, $A_{V}$, toward several IR sources in the vicinity of NGC 2024. These source positions are displayed in section 4.3.4. Using the extinction given for these positions, the $H_{2}$ column densities are derived assuming 
the ratio of extinction to the $B-V$ color excess $R=A_{V} / E(B-V)=3.1$ (Mitchell et al., 2001) and the relationship between the $H_{2}$ column density and extinction to be,

$$
N_{A_{V}}=1.87 \times 10^{21} \mathrm{~cm}^{-2} \mathrm{~A}_{\mathrm{V}}
$$

from Mitchell et al. (2001). The column densities are also derived at the same positions from the $850 \mu \mathrm{m}$ emission data from Johnstone et al. (2006) using the expression given by Fissel et al. (2007),

$$
N(850 \mu m)=3.89 \times 10^{22} S_{850}\left(\frac{\kappa_{850}}{0.02 \mathrm{~cm}^{2} \mathrm{~g}^{-1}}\right)^{-1}\left[\exp \left(\frac{17 K}{T_{d}}\right)-1\right] \mathrm{cm}^{-2}
$$

where the temperature in the expression was approximated for each individual source. This was done by considering the $\mathrm{H}_{2} \mathrm{CO}$ derived kinetic temperatures, and taking into account the distance between the source position and the ridge. The $850 \mu \mathrm{m}$ flux at each position is determined by doing photometry (in GAIA) on the map from Johnstone et al. (2006). The positions of the IR sources are labeled with their source IDs used by Haisch et al. (2001) in Fig. 4.11. The column densities derived for some of the positions in the vicinity of NGC 2024 from the above two methods are presented in Table 3.8. The comparison of the two derived sets of $\mathrm{H}_{2}$ column densities and the consequences of these results are discussed in section 4.3.4.

\subsection{Fractional Abundance}

In many molecular studies, a constant fractional abundance is assumed for the chemical species to derive $\mathrm{H}_{2}$ column densities from molecular observations. Fractional abundance is typically the ratio of the density of a species over the density of $H_{2}$. When a fractional abundance is assumed, the molecular density from observations is the only necessary parameter to derive the $H_{2}$ density. This may be deceptive, however, since the assumed fractional abundances are often found for one specific region, and may be different for the evaluation of a different region. Even within the same region, the fractional abundance may not remain constant, especially for very dense cores. A more realistic approach is to reverse the process, by considering a separate measurement of $\mathrm{H}_{2}$ column densities. Using this in combination with derived molecular column 
Table 3.8: $H_{2}$ Column Densities from Extinction and $850 \mu \mathrm{m}$

\begin{tabular}{c|cccccc}
\hline \hline Source ID & RA & Dec & $\begin{array}{c}\mathrm{T}^{b} \\
{[\mathrm{~K}]}\end{array}$ & $\begin{array}{c}A_{V}{ }^{c} \\
N_{\left(H_{2}\right)_{850 \mu m}{ }^{d}} \\
{\left[\mathrm{~cm}^{-2}\right]}\end{array}$ & $\begin{array}{c}N\left(H_{2}\right)_{A_{V}} \\
{\left[\mathrm{~cm}^{-2}\right]}\end{array}$ \\
\hline 2 & $05: 41: 45.79$ & $-01: 54: 39.05$ & 40 & 8.7 & $5.4 \times 10^{21}$ & $1.6 \times 10^{22}$ \\
4 & $05: 41: 39.04$ & $-01: 52: 09.74$ & 40 & 14 & $2.7 \times 10^{21}$ & $2.6 \times 10^{22}$ \\
14 & $05: 41: 37.22$ & $-01: 53: 15.51$ & 40 & 0.8 & $1.2 \times 10^{21}$ & $1.5 \times 10^{22}$ \\
26 & $05: 41: 36.19$ & $-01: 54: 26.83$ & 40 & 7.5 & $1.0 \times 10^{21}$ & $1.4 \times 10^{22}$ \\
33 & $05: 41: 38.27$ & $-01: 50: 40.88$ & 40 & 9.4 & $5.8 \times 10^{21}$ & $1.8 \times 10^{22}$ \\
45 & $05: 41: 39.20$ & $-01: 54: 16.15$ & 50 & 12 & $1.2 \times 10^{22}$ & $2.2 \times 10^{22}$ \\
53 & $05: 41: 36.80$ & $-01: 54: 00.78$ & 40 & 5.6 & $3.5 \times 10^{21}$ & $1.0 \times 10^{22}$ \\
58 & $05: 41: 44.38$ & $-01: 55: 24.73$ & 80 & 8.8 & $5.2 \times 10^{22}$ & $1.6 \times 10^{22}$ \\
61 & $05: 41: 30.11$ & $-01: 53: 36.02$ & 50 & 11 & $9.1 \times 10^{21}$ & $2.1 \times 10^{22}$ \\
73 & $05: 41: 44.79$ & $-01: 54: 37.06$ & 70 & 11 & $4.9 \times 10^{22}$ & $2.1 \times 10^{22}$ \\
74 & $05: 41: 45.00$ & $-01: 54: 07.57$ & 60 & 43 & $1.2 \times 10^{22}$ & $8.0 \times 10^{22}$ \\
80 & $05: 41: 41.89$ & $-01: 54: 25.95$ & 65 & 12 & $2.1 \times 10^{22}$ & $2.2 \times 10^{22}$ \\
88 & $05: 41: 53.88$ & $-01: 55: 16.82$ & 40 & 9.8 & $6.4 \times 10^{21}$ & $1.8 \times 10^{22}$ \\
92 & $05: 41: 45.98$ & $-01: 55: 03.04$ & 60 & 14 & $1.9 \times 10^{22}$ & $2.6 \times 10^{22}$ \\
98 & $05: 41: 50.23$ & $-01: 57: 45.15$ & 40 & 6.1 & $1.4 \times 10^{22}$ & $1.1 \times 10^{22}$ \\
\hline
\end{tabular}

\footnotetext{
${ }^{a}$ Source IDs correspond to the same IDs from Haisch et al. (2001)

${ }^{b}$ Temperatures at IR source positions estimated using $\mathrm{H}_{2} \mathrm{CO}$ derived temperatures at FIR core positions

${ }^{c}$ Visual extinction towards mid-IR sources given by Haisch et al. (2001)

${ }^{d} 850 \mu \mathrm{m}$ SCUBA data from Johnstone et al. (2006)
}

densities, it is possible to obtain directlychemical fractional abundances for specific positions. Here, the column densities derived for $\mathrm{H}_{2} \mathrm{CO}$ and $\mathrm{HCO}^{+}$are used with the column densities derived from sub-mm dust observations [850 $\mu \mathrm{m}$ from Johnstone et al. (2006)] for the FIR core positions to get $\mathrm{H}_{2} \mathrm{CO}$ and $\mathrm{HCO}^{+}$ fractional abundances. Johnstone et al. (2006) used $850 \mu \mathrm{m}$ data to derive properties of dense dust clumps in the vicinity of NGC 2024. Using the $21^{\prime \prime}$ beam convolved fluxes given by Fissel et al. (2007), $H_{2}$ column densities are found from Eqn. 3.17 for each FIR position. The result is presented in Table 3.9. The $\mathrm{H}_{2} \mathrm{CO}$ column densities used for the abundance ratio calculations came from the derivations of the $3_{22} \rightarrow 2_{21}$ transition (shown in Table 3.4), and the $\mathrm{HCO}^{+}$column densities came from multiplying the $\mathrm{H}^{13} \mathrm{CO}^{+}$ column densities by the assumed abundance ratio for $\mathrm{HCO}^{+} / \mathrm{H}^{13} \mathrm{CO}^{+}$.

The calculated $\mathrm{H}_{2} \mathrm{CO}$ fractional abundances range between $2 \times 10^{-9}-5 \times 10^{-9}$. These values agree well with a number of $\mathrm{H}_{2} \mathrm{CO}$ fractional abundances in previous literature. In regions of massive star formation, with warm temperatures $\left(60\right.$ to $90 \mathrm{~K}$ ), the fractional abundance of $\mathrm{H}_{2} \mathrm{CO}$ has been observed to be a few $\times 10^{-9}$ (van der Tak et al., 2000; Schreyer et al., 2002; Doty et al., 2002). Higher values have been found, however, for instance, in TMC-1 and L134N, where the observed fractional abundance was $\cong 2 \times 10^{-8}$ (van 
Table 3.9: Fractional Abundance for $\mathrm{H}_{2} \mathrm{CO}$ and $\mathrm{HCO}^{+}$at FIR Core Positions

\begin{tabular}{l|ccc}
\hline Source & $\begin{array}{c}N\left(\bar{H}_{2}\right)_{850 \mu m} \\
{\left[\mathrm{~cm}^{-2}\right]}\end{array}$ & \multicolumn{2}{c}{ Abundances $^{a}$} \\
\cline { 3 - 4 } & $\frac{\mathrm{H}_{2} \mathrm{CO} b}{\mathrm{H}_{2}}$ & $\frac{\mathrm{HCO}^{+} c}{\mathrm{H}_{2}}$ \\
\hline FIR 1 & $1.4 \times 10^{23}$ & $5.0 \times 10^{-9}$ & $6.5 \times 10^{-10}$ \\
FIR 2 & $2.2 \times 10^{23}$ & $3.9 \times 10^{-9}$ & $6.9 \times 10^{-10}$ \\
FIR 3 & $3.2 \times 10^{23}$ & $2.2 \times 10^{-9}$ & $4.0 \times 10^{-10}$ \\
FIR 4 & $2.5 \times 10^{23}$ & $2.5 \times 10^{-9}$ & $4.3 \times 10^{-10}$ \\
FIR 5 & $4.3 \times 10^{23}$ & $2.3 \times 10^{-9}$ & $7.1 \times 10^{-10}$ \\
FIR 6 & $2.2 \times 10^{23}$ & $2.6 \times 10^{-9}$ & $5.4 \times 10^{-10}$ \\
FIR 7 & $2.6 \times 10^{23}$ & $2.3 \times 10^{-9}$ & $3.2 \times 10^{-10}$ \\
\hline
\end{tabular}

\footnotetext{
${ }^{a} \mathrm{H}_{2}$ column densities derived from SCUBA $850 \mu \mathrm{m}$ data of Johnstone et al. (2006)

${ }^{b} \mathrm{H}_{2} \mathrm{CO}$ fractional abundance obtained from $\mathrm{H}_{2} \mathrm{CO}$ column densities derived using $3_{22} \rightarrow 2_{21}$ transition.

${ }^{c} \mathrm{HCO}^{+}$fractional abundances derived assuming a fixed abundance ratio for $\mathrm{HCO}^{+} / \mathrm{H}^{13} \mathrm{CO}^{+}$(Table 3.7).
}

Dishoeck et al., 1993). This study focused on cold, dark clouds, and may not be applicable to the dense, warm ridge of NGC 2024 .

The fractional abundances of $\mathrm{HCO}^{+} / \mathrm{H}_{2}$ are given in Table 3.9 to be approximately $5 \times 10^{-10}$. In comparison with other $\mathrm{HCO}^{+}$fractional abundance observations, this value is relatively low. van Dishoeck et al. (1993) gave $\mathrm{HCO}^{+}$abundances around $7 \times 10^{-9}$ for cold dark clouds in TMC-1 and L134N. For a region more resembling the NGC 2024 ridge, Blake et al. (1987) found an upper limit for the fractional abundance to be around $10^{-9}$, which is greater by a factor of 2 . Similar values $\left(1 \times 10^{-9}-5 \times 10^{-9}\right)$ were found by Helmich (1996) for IR sources in the W3 molecular gas, which is a massive star forming region. The values derived in this thesis are lower than the presented molecular regions, but there are several feasible explanations for the possible cause in the differences. One possible factor comes from the assumption which was made that $\mathrm{HCO}^{+}$and $\mathrm{H}^{13} \mathrm{CO}^{+}$had the same excitation temperature. This was forced in order to calculate the optical depth. Another likelihood is that the assumed abundance ratio of $\mathrm{HCO}^{+} / \mathrm{H}^{13} \mathrm{CO}^{+}$was incorrect, and may have been smaller. These explanations are discussed further in Section 4.2 for the comparison between the $\mathrm{HCO}^{+}$derived excitation temperatures and the $\mathrm{H}_{2} \mathrm{CO}$ derived kinetic temperatures. 


\section{Discussion}

\subsection{VARIATION OF PHYSICAL PROPERTIES THROUGHOUT RIDGE}

The temperature and density distribution across a core can unravel information concerning the object's present stage of core/star formation. For instance, a class -1 source in an isolated region should reveal a low temperature center surrounded by warmer envelopes. The core's only source of heating comes from the interstellar radiation field (ISRF) due to the absence of a central stellar source (Evans, 2003; Di Francesco et al., 2001). The density of the core should also be radially dependent by a power law $\left(n \propto r^{-2}\right)$ as suggested for singular isothermal spheres by Shu (1977). Observations have shown that this is not always the case, especially for clustered environments, and that the central profiles exhibit a flattened or plateaued distribution, whereas the outer radii show the expected steeper fall off (Di Francesco et al., 2001). This density distribution has been predicted for the inside-out collapse, where the inner radii are expected to show a power law of $-\frac{3}{2}$ (Young and Evans, 2005). The temperature and density distributions of cores in clustered regions may not show the same features as isolated regions, and further observations are needed to constrain the properties of clustered cores. Hence an observational test of the temperature and density variation can reveal the development of the core in various ways.

\subsubsection{TEMPERATURE}

The results of the temperature derivations show that the FIR cores have very warm temperatures, ranging from 46 to $85 \mathrm{~K}$ for the $3_{03} \rightarrow 2_{02} / 3_{22} \rightarrow 2_{21}$ ratios. The temperatures derived from the $5_{05} \rightarrow 4_{04} / 5_{23} \rightarrow 4_{22}$ ratios give a wider range, from 24 to $116 \mathrm{~K}$ for the cores, but these temperatures have larger uncertainties as previously discussed. Regardless of which set of temperatures is being considered, they are much higher than the cold dust temperatures originally presented by Mezger et al. (1992), and more in agreement with recent temperature studies.

The cuts of constant declination across the FIR cores (presented in Table 3.1) show that there is little variation in temperature between each FIR core and its immediate surroundings. In general, from Table 

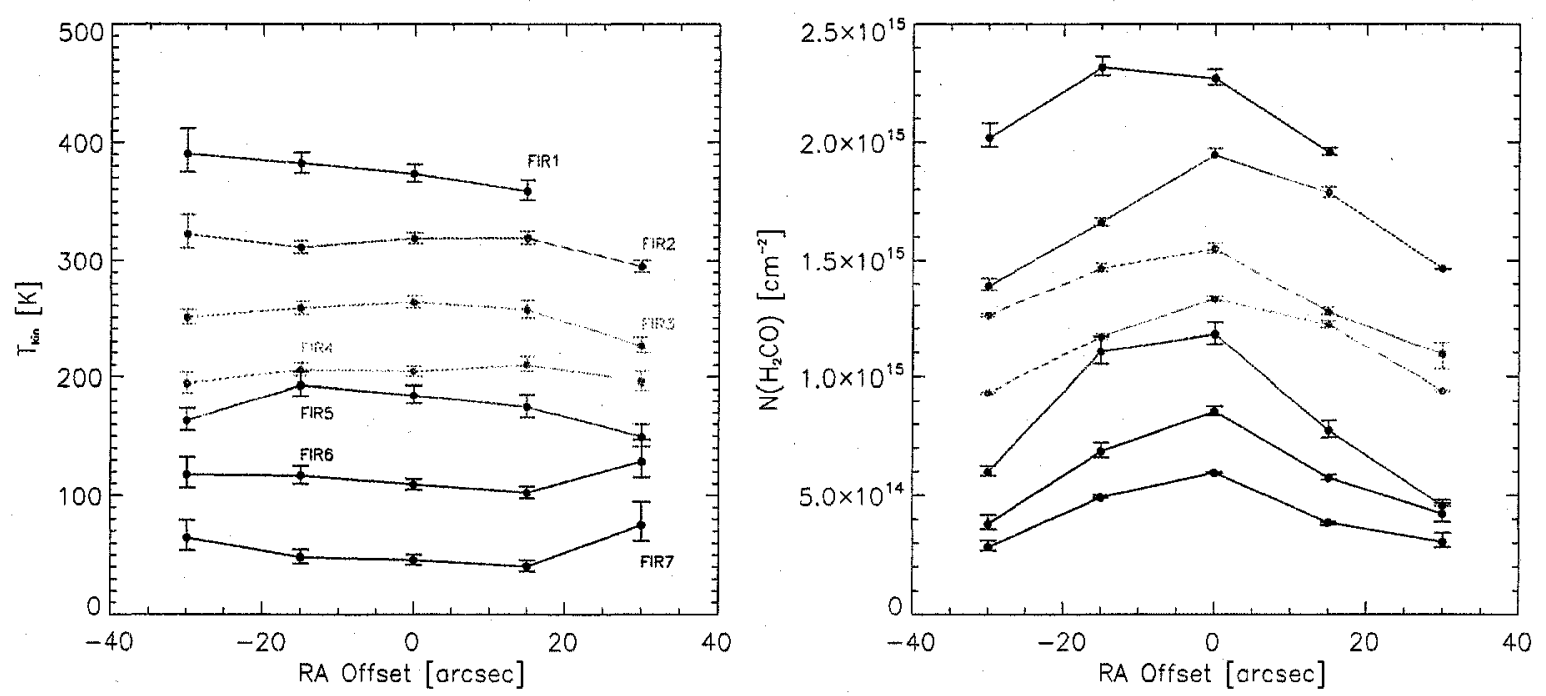

Figure 4.1: Variation of temperature derived from $\mathrm{H}_{2} \mathrm{CO}$ line ratio $3_{03} \rightarrow 2_{02} / 3_{22} \rightarrow 2_{21}$ (left) and formaldehyde column density derived from $\mathrm{H}_{2} \mathrm{CO} 3_{02} \rightarrow 2_{02}$ line (right) across FIR cuts. The cuts are in order from FIR 1 to FIR 7 (top to bottom). Each cut is arbitrarily offset to display only its variations in comparison with the other cuts.

2.4, the integrated intensities measured at the positions of the FIR cores are the largest along the cuts, in some cases significantly higher (FIRs 4-6). But the temperature depends on the intensity ratios, and these values varied little (from Eqn. 3.1). Fig. 4.1 (left) illustrates this point, showing that the temperature remains constant along the cuts, and there is no consistent trend from one FIR core cut to another.

The lack of evidence that the cores have significantly higher temperatures than their surrounding material weakens the claim of internal heating within the FIR cores. Lack of internal heating suggests that the cores are not being heated by embedded sources, and therefore do not contain massive protostars. The core centers also fail to show cooler temperatures than their surroundings, which makes it difficult to comment on whether they are being heated primarily by an outside source. Schulz et al. (1991) - in their study of the FIR cores using $C S$ excitation-found that the gas temperatures decreased with increasing column density towards the core centers found in this study, and they claimed that the main source of heating must be located outside. The warm core centers suggest that the clumpiness of the cloud allows external radiation to reach core centers. 


\subsubsection{DENSITY}

The density estimates $n\left(H_{2}\right)$ obtained from LVG models using the $\mathrm{H}_{2} \mathrm{CO}$ line ratio $3_{03} \rightarrow 2_{02} / 3_{22} \rightarrow 2_{21}$ (Table 3.3) show that the FIR core positions tend to display peak densities within the cuts. The FIR cores all have densities of $\sim 2 \times 10^{6} \mathrm{~cm}^{-3}$, with little deviation in density from one FIR core to another. It is difficult to quantify the significance of the variations in densities across cuts since these values were only roughly estimated. Nonetheless, it is probably safe to say that the cores have higher densities than their surrounding material. The column density calculations of the FIR core cuts derived from $\mathrm{H}_{2} \mathrm{CO}$ also show this, as expected. The variation of column densities derived using the $\mathrm{H}_{2} \mathrm{CO}$ derived temperatures and the line intensities from the $\mathrm{H}_{2} \mathrm{CO}_{3} 3_{03} \rightarrow 2_{02}$ line across the FIR core cuts are shown in Fig. 4.1 (right). The center core positions peak in column densities, but they do not fall off rapidly. FIR 5 shows the largest variation in column densities, possibly implying a more advanced stage for the embedded source. The core positions in general had higher column densities, but there were more variations in the values among different FIR core positions. The variation, again, is small. For the column densities derived from $3_{03} \rightarrow 2_{02}$, the cores had the range $5.9 \times 10^{21}-1.6 \times 10^{22} \mathrm{~cm}^{-2}$, with FIR 7 being the lowest and FIR 5 being the highest. From the $3_{22} \rightarrow 2_{21}$ derivations, the column densities range from $3.0 \times 10^{22}-4.9 \times 10^{22}$ $\mathrm{cm}^{-2}$, with the lowest and highest values corresponding to the same cores as in $3_{03} \rightarrow 2_{02}$ derivations. It appears that the southern clump has slightly higher column densities than the northern clump, neglecting the lowest value for FIR 7. This can also be said for the column densities derived from $\mathrm{H}^{13} \mathrm{CO}^{+}$and $\mathrm{HCO}^{+}$. This may be revealing the spatial orientation of the northern and southern clumps.

\subsection{TEMPERATURE COMPARISON}

The kinetic temperatures derived from the $\mathrm{H}_{2} \mathrm{CO}$ line ratio $3_{03} \rightarrow 2_{02} / 3_{03} \rightarrow 2_{02}$ and the excitation temperatures derived from $\mathrm{HCO}^{+}$and $\mathrm{H}^{13} \mathrm{CO}^{+}$, as presented in Table 3.1 and 3.6, have yielded two different sets of temperatures. Although the difference is almost negligible in a few FIR cores, most temperature differences range between 20 and $40 \mathrm{~K}$. This difference is not alarming, however, since the two temperatures were derived using two different methods with different assumptions. For example, in obtaining the $\mathrm{HCO}^{+}$and $\mathrm{H}^{13} \mathrm{CO}^{+}$excitation temperatures, it was initially specified that the two species share a 
common excitation temperature. This is not necessarily so, because the difference in optical depths requires that the radiation in the two lines is produced in different gas volumes which may have different kinetic temperatures. Another assumption was an abundance ratio for $\mathrm{HCO}^{+} / \mathrm{H}^{13} \mathrm{CO}^{+}$which was used to obtain the optical depth needed for the excitation temperature derivations. With these possible sources of uncertainty in mind, the $\mathrm{H}_{2} \mathrm{CO}$ derived kinetic temperatures are more trustworthy, and prone to fewer inaccuracies. In support of the reliability of the $\mathrm{H}_{2} \mathrm{CO}$ temperatures, a comparison between the $\mathrm{H}_{2} \mathrm{CO}$ LTE and LVG derived temperatures show that they are in good agreement (Tab.3.2). With the exception of a few positions, the LTE derived temperatures agree with the LVG derived temperatures within their uncertainties. Even in the most deviant case, the LTE and LVG temperatures disagree by only $7 \mathrm{~K}$. This also suggests that LTE is a good approximation for the $\mathrm{H}_{2} \mathrm{CO}$ level populations.

\subsection{StRUCtURE AND MORPhOLOGY}

\subsubsection{Comparison With $\mathrm{H}_{2} \mathrm{CO}$ absorption}

NGC 2024 contains an H II region which only partially coincides with the entire ridge, specifically with the northern clump. This spatial coincidence allows observations of molecular gas against the H II region continuum. Crutcher et al. (1986) presented absorption observations of NGC 2024 in the $H_{2} C O 1_{10} \rightarrow 1_{11}$ line at $4.8297 \mathrm{GHz}(\lambda 6 \mathrm{~cm})$. The positions of their absorption measurements are shown in Fig. 4.2. By comparing the absorption minima with the measurements made from $\mathrm{H}_{2} \mathrm{CO}$ emission, inferences can be made about whether the two observations are tracing the same region. Fig. 4.3 shows the absorption spectra from Crutcher et al. (1986) compared with the observed formaldehyde emission spectra. The emission peaks have been inverted in the intensity direction for easier comparison with the absorption. The two sets of spectral lines appear to be tracing gases of two different velocities. For example, at the position called NCP [second from top in Fig. 4.3 (left)], the absorption minimum is at $\sim 9.2 \mathrm{~km} \mathrm{~s}^{-1}$, while the emission peak [top spectrum of Fig. 4.3 (right)] is at $\sim 10.8 \mathrm{~km} \mathrm{~s}^{-1}$. The observed differences in kinematics suggest that the absorbing and emitting formaldehyde are spatially separate, although coinciding along the observed line-of-sight. In the $3_{03} \rightarrow 2_{02}$ spectra of FIRs 1-4 (cores in the northern clump), a very faint dip can be seen at the corresponding velocities of the absorption peaks. This absorption is too faint to be clearly 
detected in any of the other lines. The absorbing gas must be in front of the $\mathrm{H} \mathrm{II}$ region and must be less dense by an order of magnitude $\left(\sim 10^{5} \mathrm{~cm}^{-3}\right)$ than the emitting region. The $\mathrm{HCO}^{+}$emission spectra show self-absorption notches at approximately the same velocity peaks as the absorption peaks from Crutcher et al. (1986) for the northern clump cores FIRs $1-4\left(v_{a b s}=9.9 \mathrm{~km} \mathrm{~s}^{-1}\right)$. Since there are no absorption studies in $\mathrm{H}_{2} \mathrm{CO}$ for the southern clump, the presence of low density foreground gas in the southern clump cannot be determined. Schulz et al. (1991) suggested that since the northern clump detected in emission was not observed in the absorption study of Crutcher et al. (1986), the northern clump is most-likely located behind the $\mathrm{H}$ II region, whereas the low density gas observed in absorption is lying in front of the $\mathrm{H}$ II region. Further discussion of the dense molecular gas relative to the H II region is given in the following section.

\subsubsection{COMPARISON WITH DUST: FIR $1300 \mu \mathrm{M}$, SCUBA 850 AND $450 \mu \mathrm{M}$}

Fig. 4.4 and Fig. 4.5 show the contours of the $\mathrm{H}_{2} \mathrm{CO} 3_{03} \rightarrow 2_{02}$ transition and the $5_{05} \rightarrow 4_{04}$ transition with the positions of the FIR cores as presented by Mezger et al. (1992). From the $3_{03} \rightarrow 2_{02}$ map, we see a fair amount of agreement between the FIR core positions and the formaldehyde peaks. Most of the FIR cores can be found within $15^{\prime \prime}$ of a $3_{03} \rightarrow 2_{02}$ peak, which is a difference of approximately two thirds of the beamwidth. FIRs 1,5 , and 7 are not clearly distinguished peaks in $3_{03} \rightarrow 2_{02}$, but this may be a matter of resolution. All FIR core positions can be located in alignment with the center of the ridge, and shows no offset in right ascension. The formaldehyde peaks of the $5_{05} \rightarrow 4_{04}$ transition map-observed in higher resolution-agree more closely in position with the FIR core positions. All FIR core positions lie within 5" of a formaldehyde peak, which is one third of the beamwidth. A peculiarity is that there are at least three prominent formaldehyde peaks that were not detected in dust emission by Mezger et al. $(1988 ; 1992)$ : one between FIR 2 and FIR 3, and two just south of FIR 4. The higher resolution $5_{05} \rightarrow 4_{04}$ map also reveals that the structures of the formaldehyde cores are not circular, but are more complex. The peak near FIR 5 appears as though it may be a combination of two or more cores, as suggested by Wiesemeyer et al. (1997). Overall, the formaldehyde peaks of the two transitions $\left(3_{03} \rightarrow 2_{02}\right.$ and $\left.5_{05} \rightarrow 4_{04}\right)$ show good agreement with the positions of the FIR cores, displaying that the dust and molecular gas peak in the same positions.

The formaldehyde transition maps were interpolated to smooth out the smaller features mostly due 


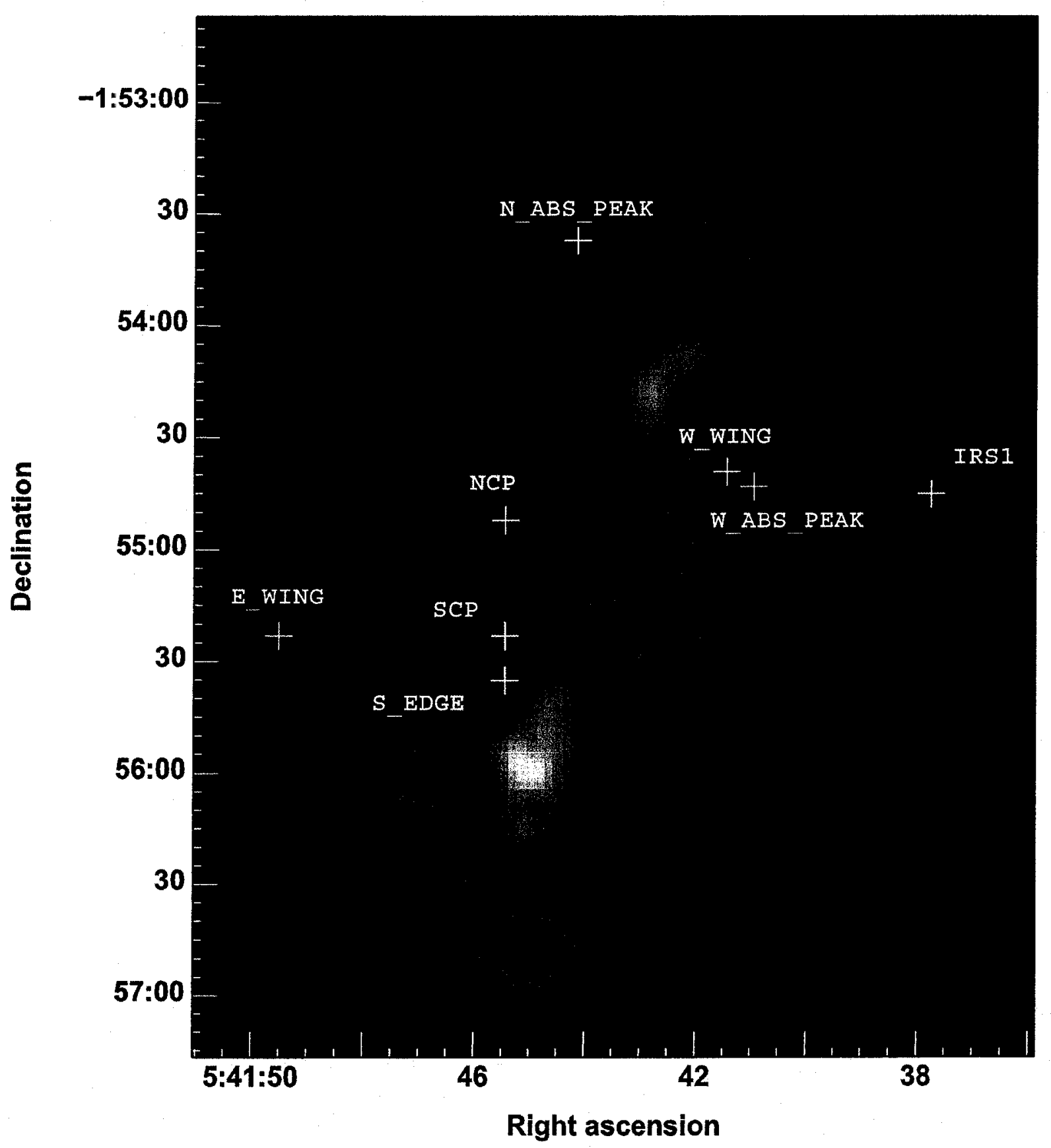

Figure 4.2: $\mathrm{H}_{2} \mathrm{CO}_{303} \rightarrow 2_{02}$ line map with labeled positions of VLA absorption measurements from Crutcher et al. (1986). 

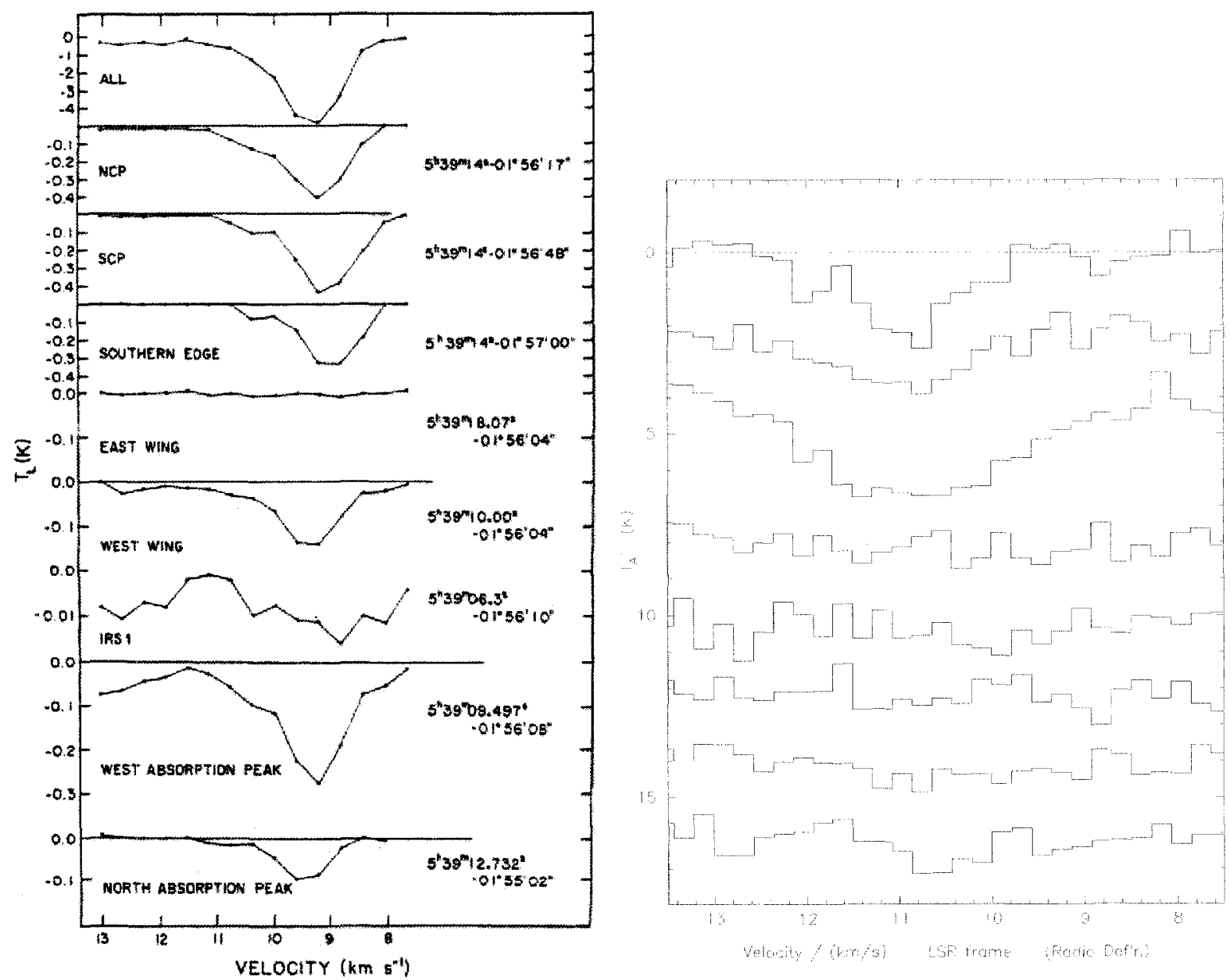

Figure 4.3: Cm absorption of $\mathrm{H}_{2} \mathrm{CO} 1_{10} \rightarrow 1_{11}$ line at $4.8297 \mathrm{GHz}$ from Crutcher et al. (1986) (left) and inverted emission spectra from $\mathrm{H}_{2} \mathrm{CO}_{303} \rightarrow 2_{02}$ line corresponding to all eight positions (right). 
to noise and emphasize the major primary features. In the $5_{05} \rightarrow 4_{04}$ case, the contours reveal a strong resemblance to the $450 \mu \mathrm{m}$ map. Fig. 4.6 displays the contours of the $5_{05} \rightarrow 4_{04}$ map plotted over a gray scale map of $450 \mu \mathrm{m}$. Although the peaks may be shifted by a few arcseconds, the positions of the peaks are in very close agreement.

Fig. 4.7 shows the contours of the $850 \mu \mathrm{m}$ SCUBA map plotted over the formaldehyde $3_{03} \rightarrow 2_{02}$ transition map. As can be observed from the comparisons between the SCUBA contours and the formaldehyde transition, the peaks loosely correspond to each other, but the differences are noticeable. In the northern clump, the formaldehyde peaks are generally slightly south of the SCUBA peaks. The clearest example is FIR 4: the formaldehyde counterpart is $15-20^{\prime \prime}$ offset in the south direction from the SCUBA counterpart. The misalignment of the position is peculiar, but is perhaps revealing the presence of the unipolar outflow in FIR 4 (Moore and Yamashita, 1995) although the direction of the outflow was approximated to be along southeast-northwest projected orientation instead of directly north-south. This inference was made due only to the detection of a small near-IR reflection nebula and the previous cases in which such types were usually associated with energetic molecular outflows. Further inspection of the outflow would be needed to clearly identify it as the cause of the difference between the positions of the formaldehyde emission peak and in the sub-mm SCUBA peak. In formaldehyde, FIR 2 and FIR 3 counterparts appear to be blended into one strong peak in between the two positions, whereas the SCUBA peak appears slightly more spatially correlated with FIR 3, though it is still difficult to distinguish the separation between FIR 2 and FIR 3. In the southern clump, the peaks are more in agreement. The positions of the peaks are more closely aligned between the formaldehyde map and the SCUBA contours. One difference to note is that the strongest peak in each case is different from the other. The strongest SCUBA peak appears at FIR 5, whereas in formaldehyde, the strongest emission peak appears more associated with FIR 6 . In both cases, FIR 7 is not clearly distinguishable, but rather appears as an extension of FIR 6 . Overall, the more diffuse outer boundary of the formaldehyde map appears shifted slightly southward in comparison to the SCUBA contours. This is more clearly observable in the southern clump. Why aren't the formaldehyde emission peaks aligned with the submm SCUBA peaks? This is perplexing because dust and molecular gas are expected to reside co-spatially. In reality, maps of molecular emission and dust emission often show major differences in detail. In fact, maps of the same region in different molecular emission lines (e.g. 


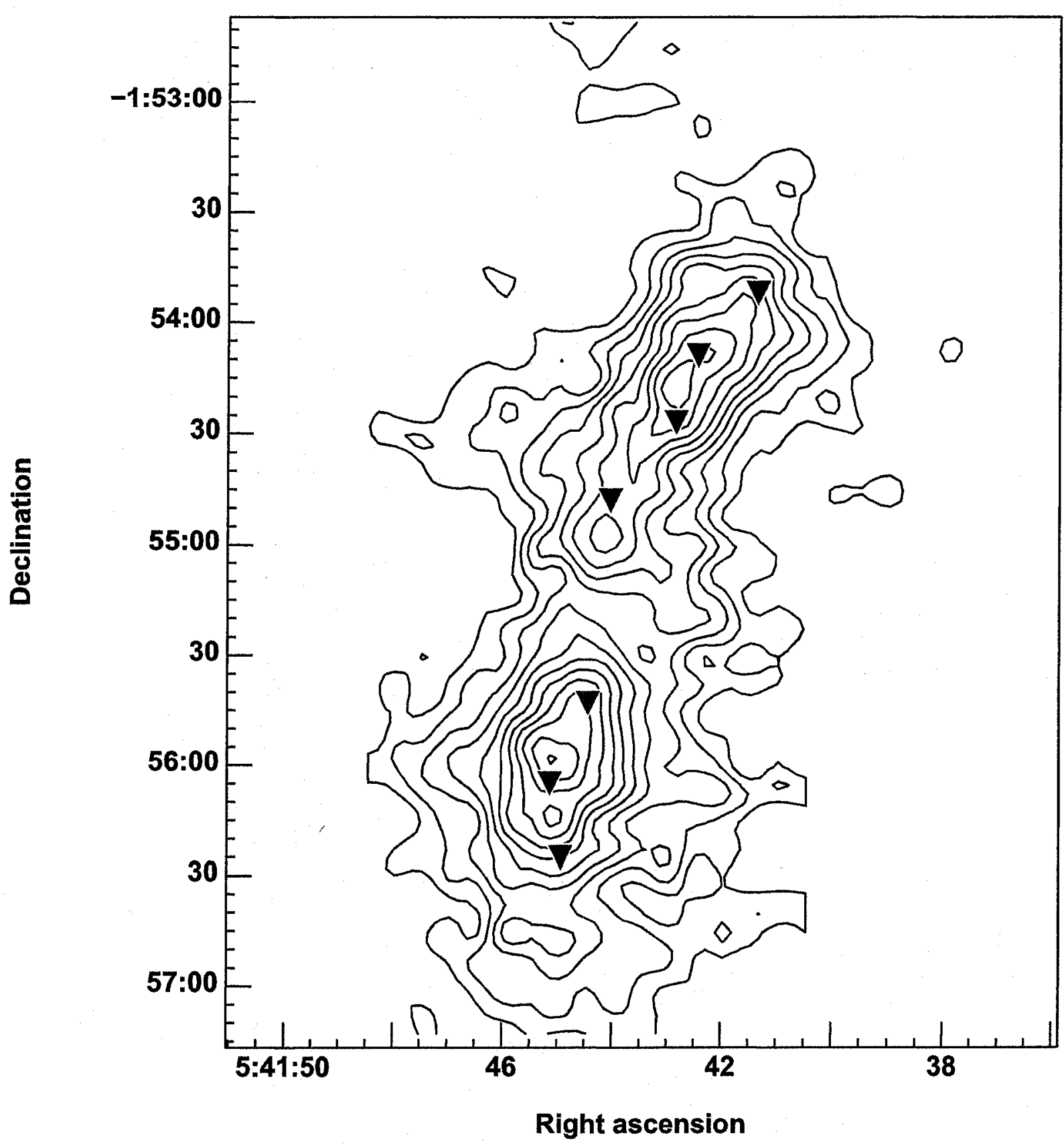

Figure 4.4: Contour map of $\mathrm{H}_{2} \mathrm{CO}_{303} \rightarrow 2_{02}$ transition. The positions of the FIR cores are indicated by red triangles. The contours represent intensities between 2 to $12 \mathrm{~K} \mathrm{~km} \mathrm{~s}^{-1}$, at intervals of $1 \mathrm{~K}$ $\mathrm{km} \mathrm{s}^{-1}$. 


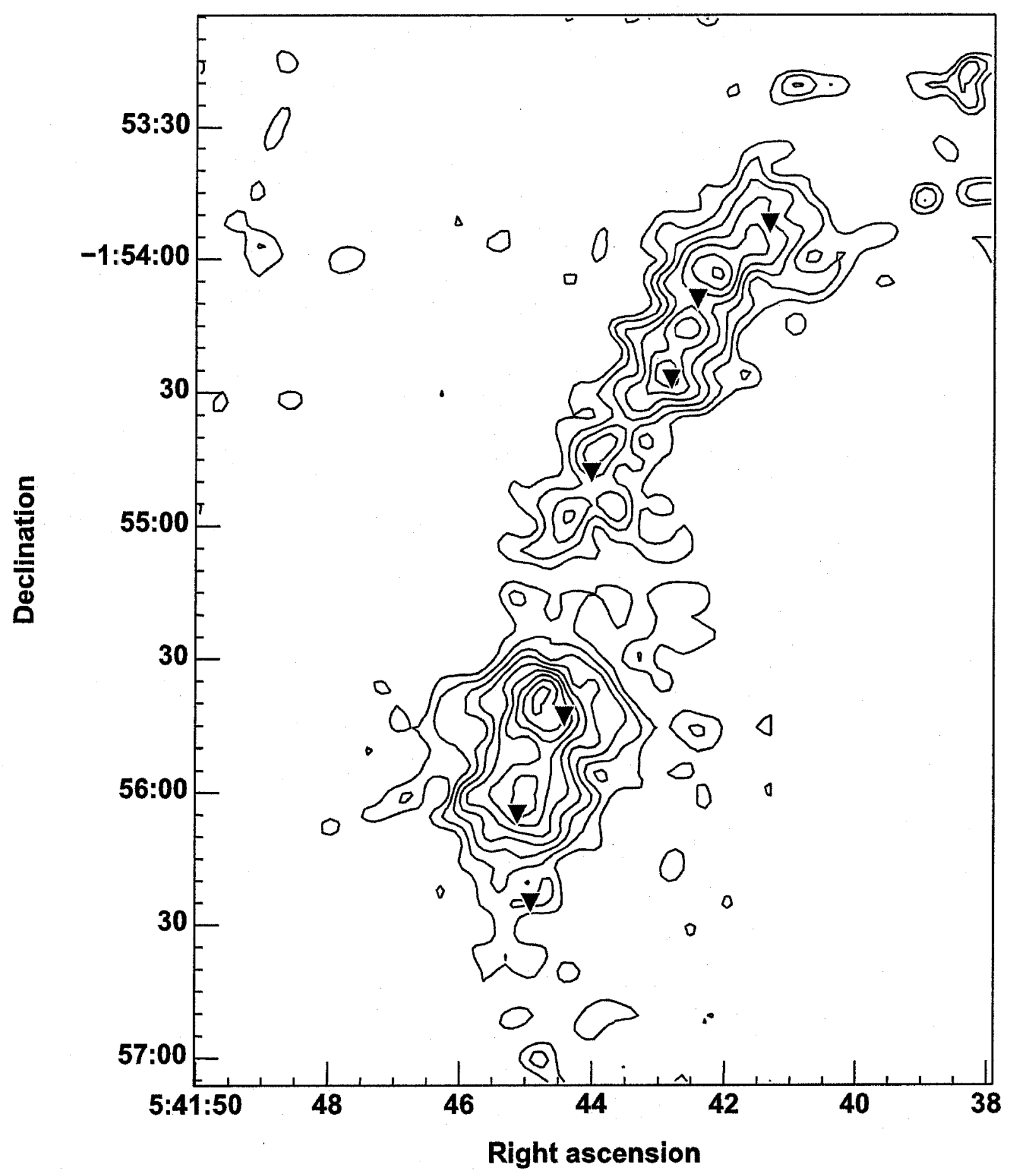

Figure 4.5: Contour map of $\mathrm{H}_{2} \mathrm{CO}_{505} \rightarrow 4_{04}$ transition. The positions of the FIR cores are indicated by red triangles. The contours represent intensities between 2 to $10 \mathrm{~K} \mathrm{~km} \mathrm{~s}^{-1}$, at intervals of $1 \mathrm{~K}$ $\mathrm{km} \mathrm{s}^{-1}$. 




Figure 4.6: Contours of interpolated $\mathrm{H}_{2} \mathrm{CO} 5_{05} \rightarrow 4_{04}$ line plotted over gray scale map of $450 \mu \mathrm{m}$. The contours of the $5_{05} \rightarrow 4_{04}$ line display intensities between 1 and $10 \mathrm{~K} \mathrm{~km} \mathrm{~s}^{-1}$ at steps of $1 \mathrm{~K}$ $\mathrm{km} \mathrm{s}^{-1}$. 
$\mathrm{HCO}^{+}, \mathrm{N}_{2} \mathrm{H}^{+}, \mathrm{CS}$ ) often exhibit peaks at different positions. One proposed explanation for this curiosity is attributed to molecular excitation issues, and how the temperature for excitation varies from one species to another. Another possibility is that variations in the fractional abundances from place to place may contribute to the observed peak positions in different molecules.

\subsubsection{COMPARISON WITH OTHER MOLECULAR STUDIES}

Figs.4.8 and 4.9 show the contours of the $\mathrm{H}_{2} \mathrm{CO}_{303} \rightarrow 2_{02}$ transition map and the $5_{05} \rightarrow 4_{04}$ transition map with the positions of the FIR cores presented by Mezger et al. (1992), and CS peaks presented by Schulz et al. (1991). The only strong correlation with the $3_{03} \rightarrow 2_{02}$ peaks and the CS peaks appears at one peak near FIR 6. CS 2, 3, and 4 appear close to (within $15^{\prime \prime}$ of) $3_{03} \rightarrow 2_{02}$ peaks. The contours of the $5_{05} \rightarrow 4_{04}$ map show closer correlation with the CS peaks, probably due to its higher resolution. CS 2 , $3,4,5,6$, and 7 are all located within $5^{\prime \prime}$ of $5_{05} \rightarrow 4_{04}$ peaks. A major difference between $C S$ and $\mathrm{H}_{2} \mathrm{CO}$ worth pointing out is that an arc seen in CS connecting the northern and southern clumps is not apparent in either of the $\mathrm{H}_{2} \mathrm{CO}$ maps. Our $\mathrm{H}_{2} \mathrm{CO}_{5} \mathrm{~S}_{05} \rightarrow 4_{04}$ transition map also shows a number of peaks that are not seen in CS. Schulz et al. (1991) pointed out that differences between different molecular distributions most likely reflect the chemical abundance gradients inside the very high density cores.

\subsubsection{EMBEDDED IR SOURCES: COMPARISON WITH MID- AND NEAR-IR}

Haisch et al. (2000) presented a near-IR $3.4 \mu \mathrm{m}$ survey of the NGC 2024 region and detected 257 sources within $110 \operatorname{arcmin}^{2}$. The positions of these sources that had complete $J H K L$ photometry from previous studies were presented, and compared with the location of the NGC 2024 ridge. Fig. 4.10 displays the location of the near-IR sources in the vicinity of the ridge. FIRs 1,2 , and 7 have near-IR sources within $10^{\prime \prime}$, as can be observed from Fig. 4.10. However, these may well be coincidences, since the remainder of the near-IR sources in general show no association with the ridge, and there is no sign that the star cluster originated from the ridge. Hence, there is no strong evidence for embedded young stars within the FIR cores. It is worth noting again that the sources presented in the study were not complete, and there may have been sources detected that were more closely corresponding to the FIR cores but left off from the list due to lack of available $J H K L$ photometry data. 


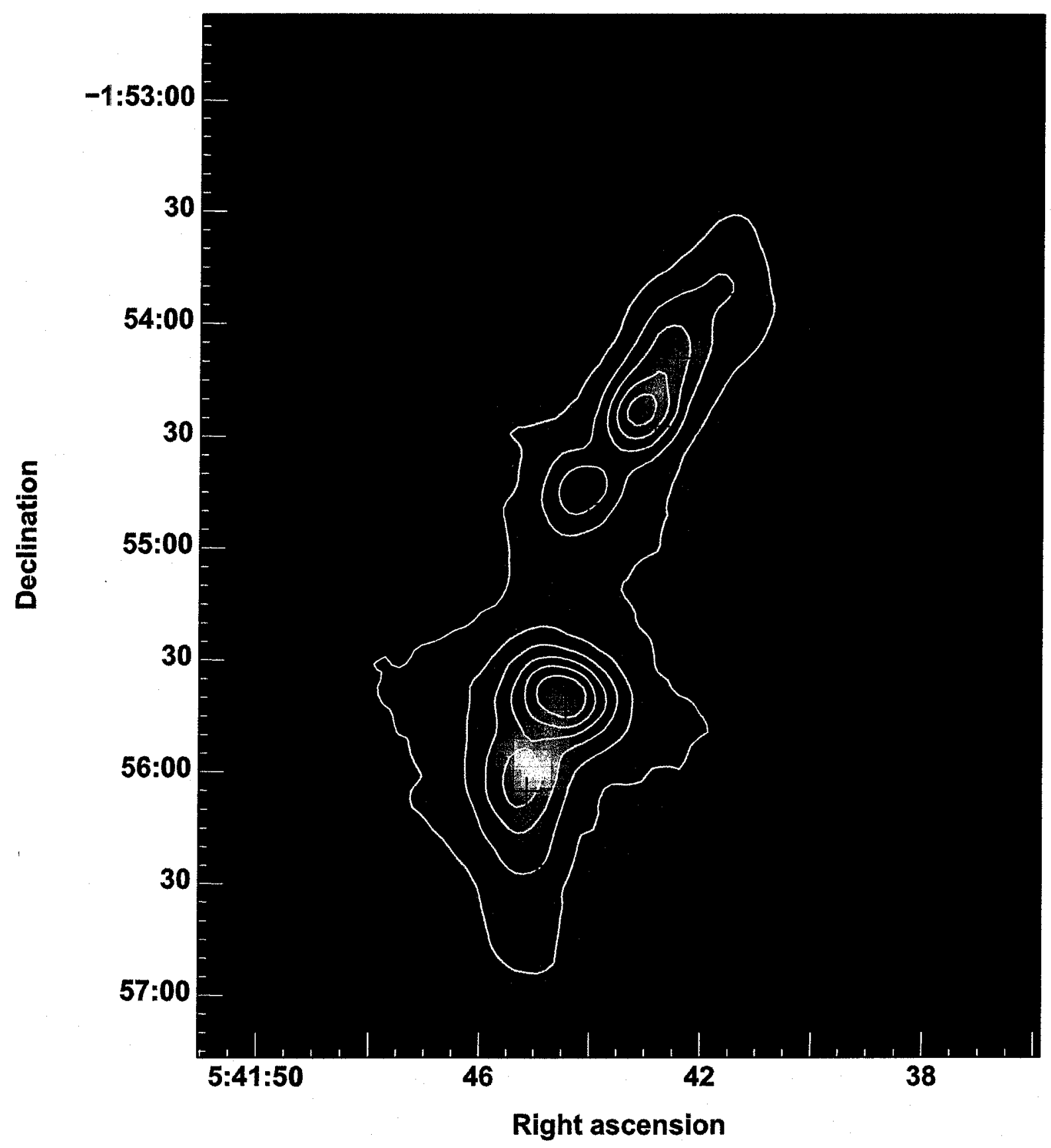

Figure 4.7: Contours of SCUBA $850 \mu \mathrm{m}$ map plotted over $\mathrm{H}_{2} \mathrm{CO}_{303} \rightarrow 2_{02}$ transition gray scale map. The $850 \mu \mathrm{m}$ contours represent intensities at $2,4,6,8,10$, and $12 \mathrm{~K} \mathrm{~km} \mathrm{~s}^{-1}$. The crosses indicate the positions of the FIR cores. 


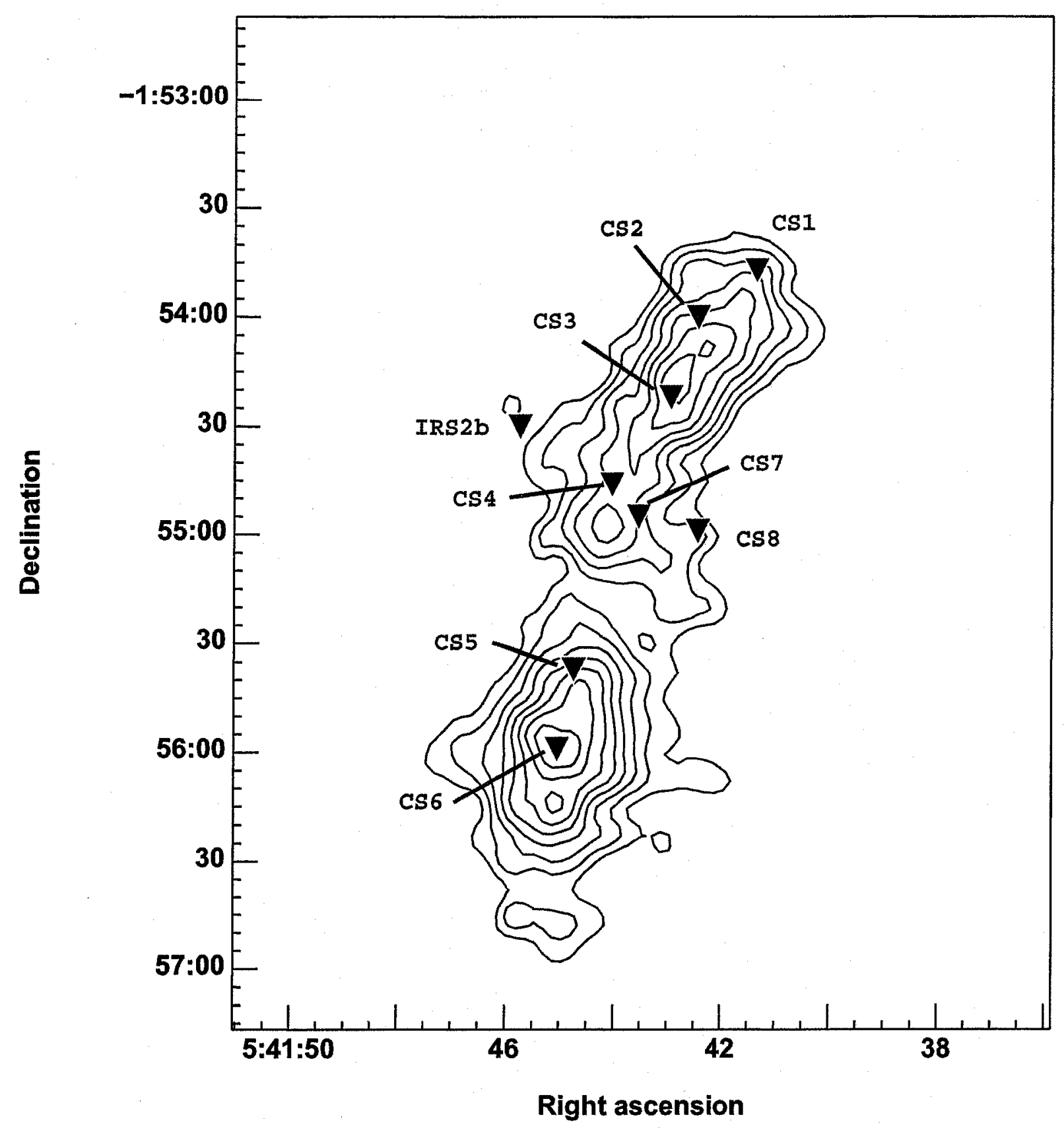

Figure 4.8: $\mathrm{H}_{2} \mathrm{CO} 3_{03} \rightarrow 202$ transition contours with positions of FIR cores, $C S$ cores from Schulz et al. (1991) and ionizing source IRS2b. The contours show intensities from 4 to $12 \mathrm{~K} \mathrm{~km} \mathrm{~s}^{-1}$ at steps of $1 \mathrm{~K} \mathrm{~km} \mathrm{~s}^{-1}$. 


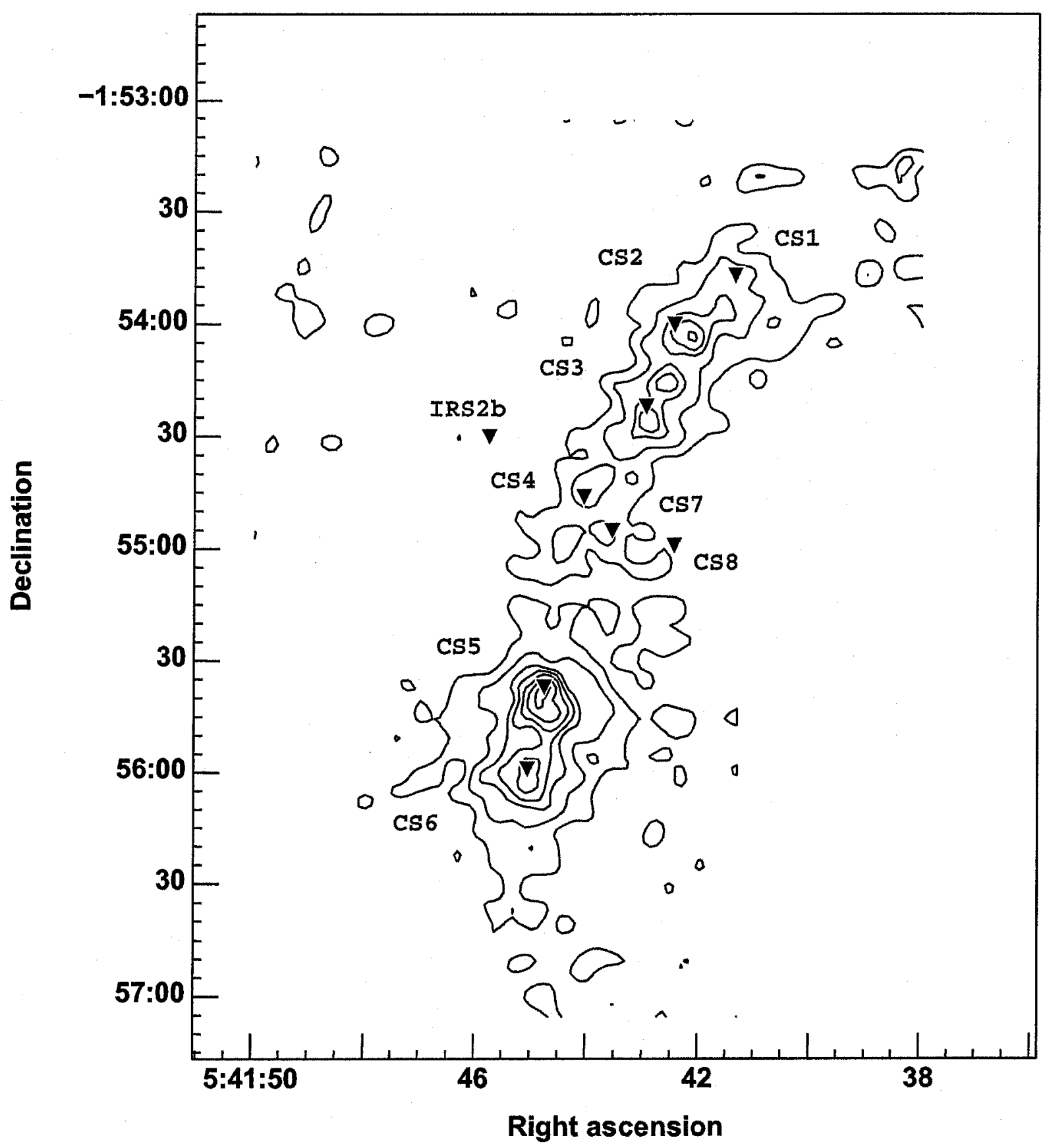

Figure 4.9: $\mathrm{H}_{2} \mathrm{CO} \mathrm{5}_{05} \rightarrow 4_{04}$ transition contours with positions of FIR cores, $\mathrm{CS}$ cores from Schulz et al. (1991) and the ionizing source IRS2b. The contours show intensities from $2,4,6,7,8,9$, and $10 \mathrm{~K} \mathrm{~km} \mathrm{~s}^{-1}$. 


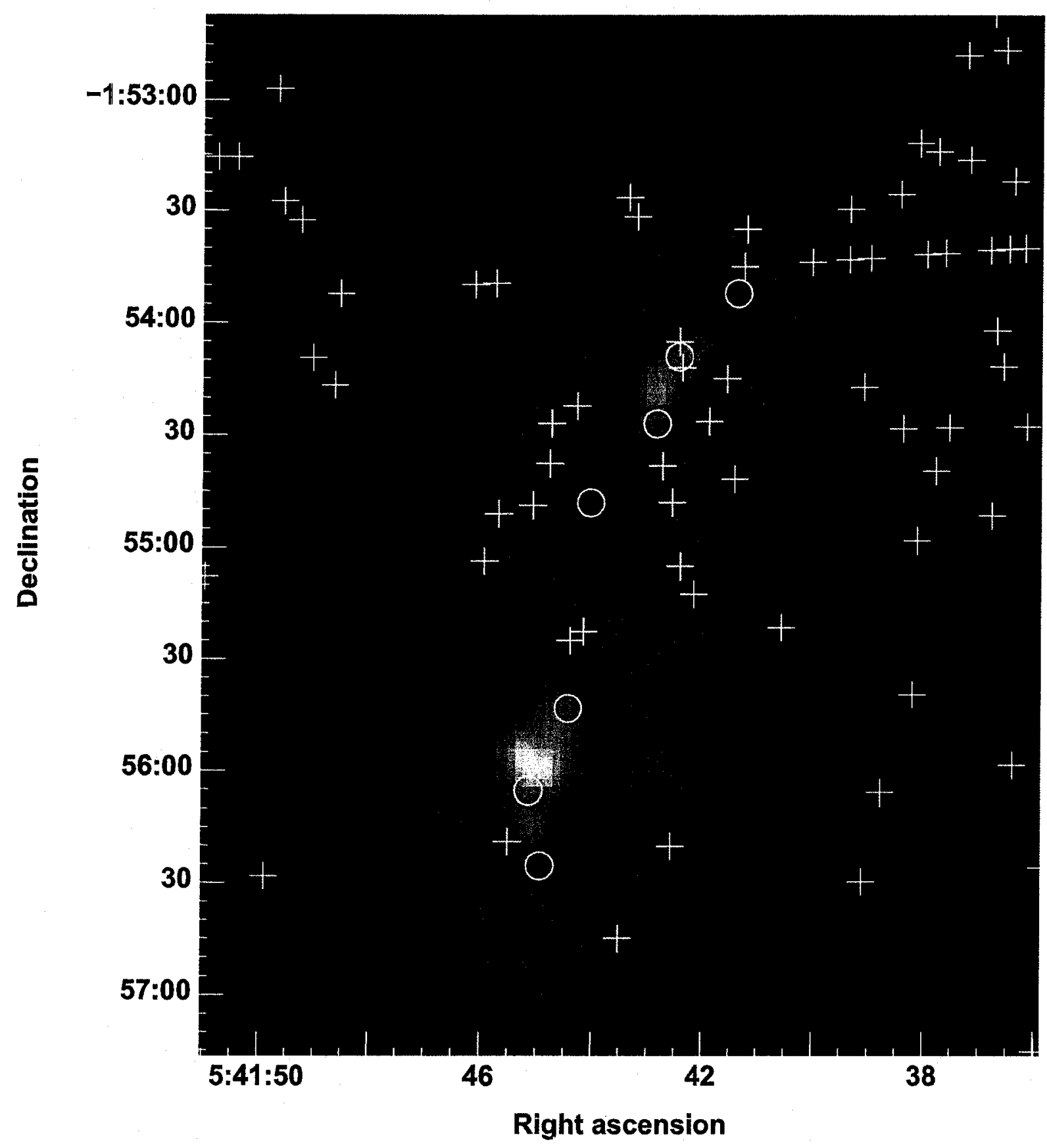

Figure 4.10: Comparison of $\mathrm{H}_{2} \mathrm{CO} 3_{03} \rightarrow 2_{02}$ map with NIR $(3.4 \mu \mathrm{m}) L$-band sources from Haisch et al. (2000). The plus signs indicate the positions of NIR sources, and the 7 open circles indicate the positions of the FIR cores. There are no exact coincidences of the NIR sources with the FIR cores. 
Haisch et al. (2001) surveyed NGC 2024 in the mid-IR at $10.8 \mu \mathrm{m}$ to find evidence for circumstellar disks around YSOs in the region. The detected sources with previous $J H K L$ photometry data were presented and used to calculate extinction towards the mid-IR sources. Fig. 4.11 shows the positions of some of the detected disk sources relative to the ridge, labeled by the source IDs assigned by Haisch et al. (2001). The objects which are excluded from the figure (source IDs $4,33,88$, and 98) are outside the map boundary. None of the detected sources have positions which coincide with the FIR cores. They are, however, close enough to the ridge to aid in the analysis of the structure of NGC 2024. In section 3.4, the visual extinctions towards the mid-IR sources are used to derive $\mathrm{H}_{2}$ column densities. These column densities are compared with $H_{2}$ column densities derived from the SCUBA $850 \mu \mathrm{m}$ data [from Johnstone et al. (2006)] at the same positions as the mid-IR sources. The result shown in Table 3.8 displays the two sets of column densities. Allowing a difference of a factor of 2 to be taken as an "agreement", the following statistics are found from the comparison. There are 11 positions where the $H_{2}$ column densities are in agreement, and four positions where the $H_{2}$ column densities derived from $850 \mu \mathrm{m}$ data are greater. The majority of the mid-IR sources have column densities in agreement with the sub-mm derived column densities. These sources are more likely to be located behind the ridge. However, four mid-IR sources show extinction derived column densities which are higher than the sub-mm derived column densities. These few stars may be embedded in their small envelopes or circumstellar disks, and may have been diluted in the beam of the SCUBA observations. There is no suggestion that any of the mid-IR sources is located in front of the ridge.

Fig. 4.12 shows the formaldehyde $3_{03} \rightarrow 2_{02}$ contours overlaid on the Midcourse Space Experiment (MSX) $8 \mu \mathrm{m}$ image. This mid-IR emission traces the dust heated by ionization, and outlines the sharply delineated "bay" to the south, which is ionization bounded. (Johnstone et al., 2006). The spatial agreement of this sharply ionized southern boundary of the HII region with the "gap" separating the northern and southern clumps of NGC 2024 has clear implications. The inclination of the ridge along our line of sight can be inferred as the northern clump tilting away from us as the southern clump is tilted towards us. The area separating the two clumps may be due to the obstruction of the sharply ionized "bay".

The $850 \mu \mathrm{m}$ study by Johnstone et al. (2006) identified discrete emission features (clumps) from NGC 2024. The detected clumps were presented, with calculated mass and radius for each source, assuming a constant temperature of $20 \mathrm{~K}$. Comparing the source positions with the positions of the FIR cores, it was 


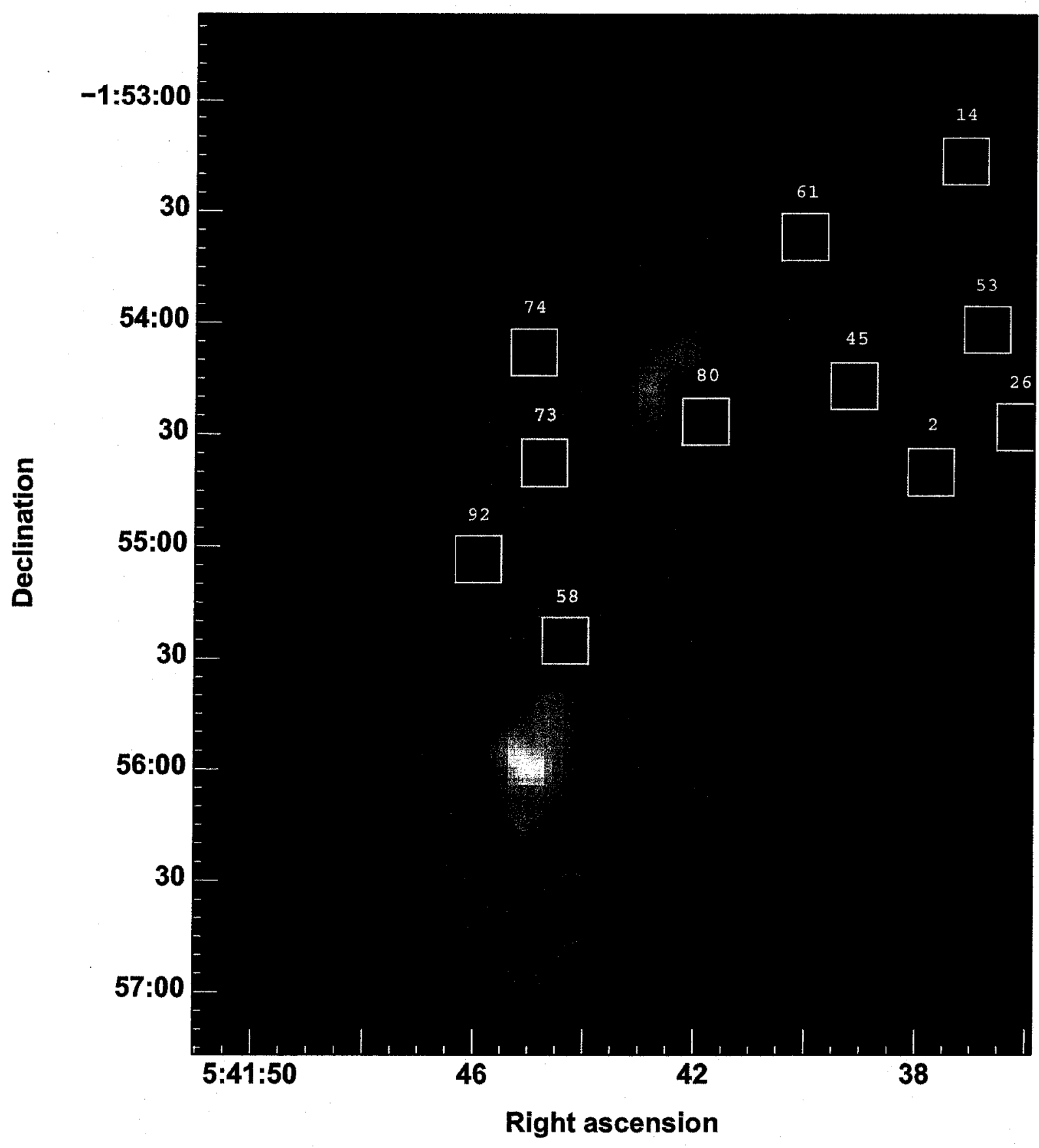

Figure 4.11: Comparison of $\mathrm{H}_{2} \mathrm{CO}_{303} \rightarrow 2_{02}$ map with MIR $(10.8 \mu \mathrm{m}) \mathrm{N}$-band sources from Haisch et al. (2001). The MIR sources are labeled using the same source IDs as Haisch et al. (2001). 


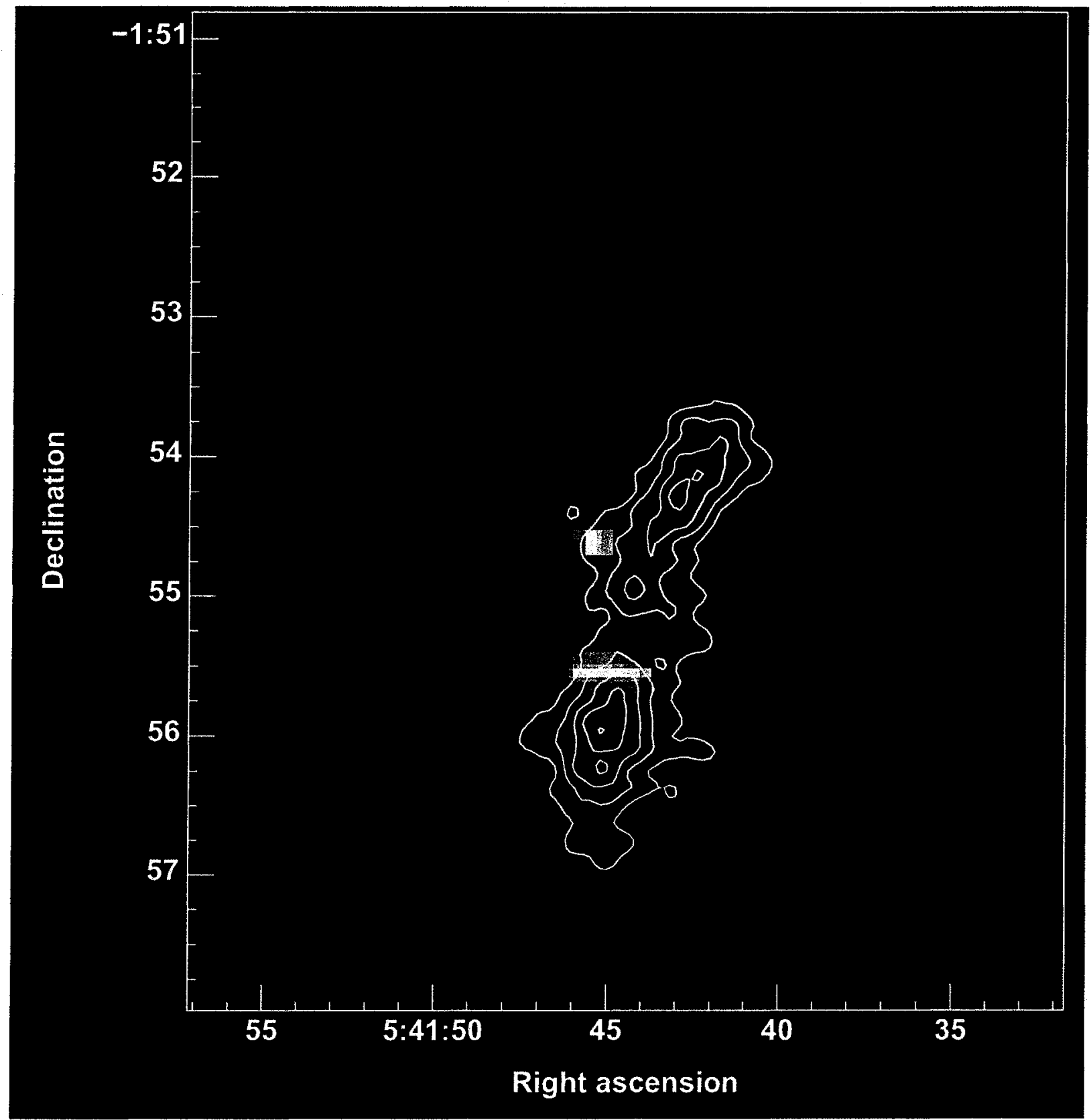

Figure 4.12: MSX $8 \mu \mathrm{m}$ with contours of $\mathrm{H}_{2} \mathrm{CO}_{303} \rightarrow 2_{02}$ line. The contours show intensities between 4 and $12 \mathrm{~K} \mathrm{~km} \mathrm{~s}^{-1}$ at steps of $2 \mathrm{~K} \mathrm{~km} \mathrm{~s}^{-1} .8 \mu \mathrm{m}$ traces the high temperature dust heated by ionization. The ionization front can be seen to coincide with the gap separating the northern clump and the southern clump. 
found that the four most massive clumps were coincident with FIR sources, specifically FIRs 3-6. Fig. 4.13 from Johnstone et al. (2006) shows the same region in various wavelengths. The bottom left panel displays the positions of the detected clumps and their sizes signified by the size of the crosses. This image clearly shows the major clumps' agreement with FIR core positions. The remaining FIR cores are undetected, but their failure to be identified may be due to the automated clump finder used and the criteria limits defined by Johnstone et al. (2006). The possibility that their spatial proximities to each other are too close and may be overlapping is another factor that may play a role in their going unnoticed.

\subsubsection{COMPARISON WITH OPTICAL}

The association of the molecular ridge with the dust is evident when comparing the contours of the formaldehyde maps with the optical image. Fig. 4.14 (top) is NGC 2024 from the DSS, with the contours indicating the various strengths of the formaldehyde $3_{03} \rightarrow 2_{02}$ transition. The DSS image takes up a much larger region than the formaldehyde contours, but it appears that the peaks of strongest formaldehyde emission coincide closely to the large central dust band obscuring the H II region. This observation strongly supports the notion that the molecular gas is cohabiting with the dust responsible for the obscuration in the foreground of the H II region. The weak extension of the northern clump formaldehyde emission to the east appears to correspond to the larger dust arm extending to the east. The inclination of the entire ridge to the north-west appears to follow the north-east tilt of the east arm of the dust. Fig. 4.14 (bottom) which shows the correlation of the $850 \mu \mathrm{m}$ contours with the optical image displays the same trends as the formaldehyde emission. The outer diffuse extension follows the same general course as the dust band. These observations suggest that the $850 \mu \mathrm{m}$ dust and the $\mathrm{H}_{2} \mathrm{CO}$ transitions are tracing the same region, their resembling shapes with the optical dust band implies that they are located in front of the H II region. The similar shapes of the dust band and the molecular ridge may merely be a coincidence, however, since the molecular ridge was not detected in the formaldehyde absorption study of Crutcher et al. (1986). 




Figure 4.13: Fig.6 from Johnstone et al. (2006). NGC 2024 at (top-left) $450 \mu \mathrm{m}$, (top-right) optical from DSS, (bottom-left) $850 \mu \mathrm{m}$ with plus signs indicating the positions of the detected clumps and sizes indicating the calculated clump masses, and (bottom-right) $8 \mu \mathrm{m}$ showing the sharply delineated south "bay". 

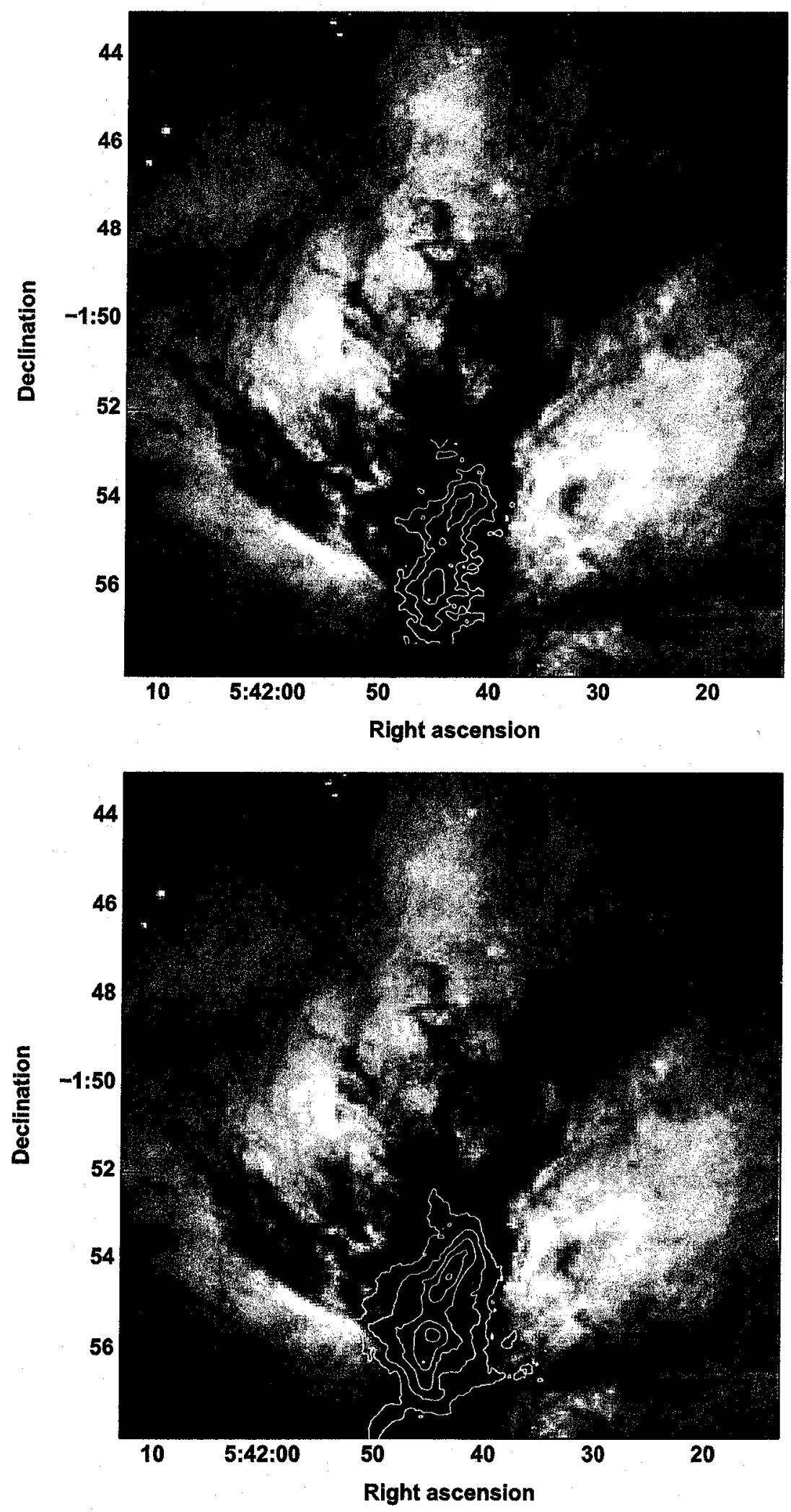

Figure 4.14: DSS image of NGC 2024 with contours of $\mathrm{H}_{2} \mathrm{CO}_{303} \rightarrow 2_{02}$ (top) and contours of SCUBA $850 \mu \mathrm{m}$ (bottom). The $\mathrm{H}_{2} \mathrm{CO}$ contours show intensities at 2,4 , and $8 \mathrm{~K} \mathrm{~km} \mathrm{~s}^{-1}$. The SCUBA contours show intensities at $0.5,1,2,4$, and $10 \mathrm{~K} \mathrm{~km} \mathrm{~s}^{-1}$. 


\subsection{StAR FORMATION}

The study of star formation has mostly been limited to the observations of isolated "field" molecular clouds, due to the limit of sensitivity and resolution of the instruments. Isolated regions are less obscured by dust, and therefore have been more intensively investigated. Isolated regions nurse the formation of low-mass stars, and the absence of massive stars-whose presence would dominate the luminosity of the lower mass stars-provides a simpler situation for observation. More recently, since the development of infrared and sub-mm instruments, the thick dust in clustered environments can be penetrated to probe the activity taking place in embedded clusters of young stars. NGC 2024 is an example of such an environment, where the center-most dense region could not be studied in detail until recently. Because we see that binary and multiple star systems are more common, it is important that we understand the nature of cluster environments and the interactions in clustered star formation. Such clustered environments provoke curiosity, since star formation must be more complicated than in isolated regions, and the influence of the surroundings must have some effect. For example, what kind of effect does it have on formation when neighboring stars are competing for the same material, and how is evolution affected by nearby massive stars blowing away gas? Questions about whether the circumstellar disks in such regions are disrupted by close-by stars have also been posed, as is the result in simulations (Bate et al., 2003). Another curiosity is that clusters give birth to stars of all different masses, and high-mass stars tend to be found surrounded by low-mass stars in clusters. Low and high-mass stars differ vastly in their formations. High-mass stars have greater densities and masses so they collapse on smaller time scales. Their luminosities and higher effective temperatures also relate to their faster evolution, making it more difficult to identify the processes for high-mass star formation. The difficulty caused is by the fact that high-mass stars are fewer in number and tend to be more embedded in the thickest regions of dust. Simulations have shown that the most massive stars tend to form in cluster centers, where there are enhanced accretion and interaction rates (Bonnell and Bate, 2002; Bonnell et al., 2003). In contrast to low-mass stars that can be found in all star-forming regions, high-mass stars are only found in Giant Molecular Clouds. Their presence is inferred, for example, by observations of masers such as $\mathrm{OH}$ and $\mathrm{H}_{2} \mathrm{O}$ which arise in outflows or circumstellar disks. In low-mass star formation, magnetic fields contribute significantly to counteract gravity, but in high-mass stars, their 
gravity is too strong and is less affected by the magnetic field.

In earlier studies of NGC 2024, the masses of the FIR cores were determined to be very massive due to the low estimated temperatures. Most studies concluded that the region was undergoing massive starformation. Mezger et al. (1992) derived masses around $10 \mathrm{M}_{\odot}$ within $5^{\prime \prime}$ of FIR cores, and Johnstone et al. (2001) found an average of $\sim 70 \mathrm{M}_{\odot}$ for FIR cores within a $21^{\prime \prime}$ beam. These studies assumed a constant dust temperature around $20 \mathrm{~K}$, which is lower than the $\mathrm{H}_{2} \mathrm{CO}$ derived temperatures. The masses of the FIR cores derived here are much lower. The highest of which was for FIR 5 (using the temperature derived from $3_{03} \rightarrow 2_{02} / 3_{22} \rightarrow 2_{21}$ ) at $3.7 \mathrm{M}_{\odot}$, far from a high-mass star. The lowest was FIR 1 at $1.2 \mathrm{M}_{\odot}$. These values are an order of magnitude less than the previously derived masses. Hence, the NGC 2024 ridge cannot be a site of massive star formation, and the possibility that the cores will form high-mass stars is much lower than previously thought.

\subsubsection{Velocity inFormation}

The channel maps at several velocity increments (Figs.2.3 and 2.5) and the molecular spectra of the FIR cores (Figs.2.9 and 2.10) presented in Chapter 2 provide clues about the velocity characteristics and the dynamics of the gas being traced. As mentioned before, the channel maps (in $3_{03} \rightarrow 2_{02}$ and $5_{05} \rightarrow 4_{04}$ ) show that the northern clump peaks at lower velocities $\left(\sim 10.5 \mathrm{~km} \mathrm{~s}^{-1}\right)$ whereas the southern clump peaks at higher velocities $\left(\sim 11.5 \mathrm{~km} \mathrm{~s}^{-1}\right)$. The spectra of the individual FIR cores show similar velocity features. FIRs 1-4 (in the northern clump) range in peak velocities from $10.2-10.6 \mathrm{~km} \mathrm{~s}^{-1}$. FIRs 5-7 (in the southern clump) all display peak velocities near $11.4 \mathrm{~km} \mathrm{~s}^{-1}$, about $1 \mathrm{~km} \mathrm{~s}^{-1}$ higher than the northern clump cores. It is unclear whether this velocity difference between the northern and southern clumps is suggesting rotation, but it may be safe to say that the two clumps are kinematically different, though weakly linked. The gap of faint emission between the northern and southern clumps is a good indicator of this. This gap corresponds spatially to the sharp ionization bound of the H II region, perhaps indicating that the northern and southern clumps may be separated by the ionized gas.

The line profiles also offer clues about the turbulent nature of the region. The FIR core spectra have

very broad lines (FWHM of $\sim 2 \mathrm{~km} \mathrm{~s}^{-1}$ ), approximately ten times the thermal line width, signifying a considerable amount of turbulence. The lines for the FIR cores in the southern clump have broader lines, 
indicating that the southern clump is more turbulent. Winds from young stars and the interaction between mołecular and ionized regions are some of the possible sources of turbulence (Shu et al., 1987). The line shapes of the cores are also suggestive of non-quiescent areas. From Fig. 2.9, it is clear that the lines are asymmetric, and non-Gaussian. More apparent in the $\mathrm{H}_{2} \mathrm{CO} 3_{03} \rightarrow 2_{02}$ and $5_{05} \rightarrow 4_{04}$ lines of FIRs 4-6 are the blue shoulders, and a weak red wing, most prominent in FIR 5 . These features are suggestive of the core gas being in motion, possibly experiencing dynamic events such as outflows. This is in agreement with evidence of outflows from previous observations: FIR 4 (Moore and Yamashita, 1995), FIR 5 (Richer et al., 1992), and FIR 6 (Richer, 1990).

Direct observation of gravitational collapse is difficult, and only indirect estimates of infall speeds are available from statistical studies (Evans, 2003). The difficulty arises from the fact that the collapse velocity is small, and easily obscured by other motions such as turbulence, thermal broadening, rotational motions, and energetic outflows. Infall is ideally observed as a double peaked profile, where the blue peak is stronger than the red. Although the intentions of observing the FIR cores in $\mathrm{HCO}^{+}$were to detect these characteristics of infall, such features could not be found, as stated in section 3.3. Instead, the $\mathrm{HCO}^{+}$spectral lines displayed red-skewed double-peaked profiles. Red profiles are most likely indicative of dense clumps in outflowing gas (Evans, 2003), which is in agreement with other observations of outflows.

\subsubsection{Stability of FIR CORES}

One of the chief questions regarding the FIR cores is whether they are contracting. The virial theorem is used to determine whether a clump is stable against collapse [e.g. Ward-Thompson et al. (2006)]. From the virial theorem, a contracting spherical cloud obeys the inequality,

$$
2 \mathcal{U}+2 \mathcal{T}+2 \mathcal{R}<\mathcal{G}+\mathcal{X}
$$

where $\mathcal{U}$ is the thermal energy, $\mathcal{T}$ is the turbulent energy, $\mathcal{R}$ is the rotational energy, $\mathcal{G}$ is the magnitude of the self-gravitational potential energy, and $\mathcal{X}$ is the contribution from external pressure. The contribution from rotational energy is assumed to be negligible, since there is no clear evidence of rotation in any of the FIR cores. Magnetic energy is also neglected as a major source of support. Although the presence of a 
magnetic field within the cores is likely, its significance in star formation is controversial. In a recent review by Bourke and Goodman (2004), it is claimed that magnetic fields are not energetically dominant during the protostellar stage. Although the magnetic field has been determined for the large scale structure of NGC 2024, it is unknown at the scale of the FIR cores. Hence, its corporation into the virial calculation is difficult, and avoided in this evaluation.

The virial stability evaluation is carried through by using the core sizes given by Johnstone et al. (2001), and the remaining physical properties (masses, temperatures, number densities) obtained from $\mathrm{H}_{2} \mathrm{CO} . \mathrm{By}$ comparing the positions of clumps found in the $850 \mu \mathrm{m}$ study with the FIR core positions, only four could be identified as clumps. The remaining cores that could not be detected as clumps were omitted from the virial stability calculations, since the properties of the cores-such as the radius and mass-were not available by the $850 \mu \mathrm{m}$ data. As a result, the virial evaluation is made for only FIRs $3,4,5$, and 6 .

The thermal energy is evaluated by using the temperatures derived from $\mathrm{H}_{2} \mathrm{CO}$,

$$
2 \mathcal{U}=\frac{3 M k_{B} T}{m}
$$

where $M$ is the mass, $k_{B}$ is the Boltzmann constant, $T$ is the temperature, and $m$ is the mean molecular mass. In the calculations, the gas within the core is assumed to be isothermal and monatomic, so $m=$ $1.67 \times 10^{-24} \mathrm{~g}$.

The turbulent energy is given by,

$$
2 \mathcal{T}=\frac{3 M \Delta V_{F W H M}^{2}}{8 \ln (2)}
$$

where $\Delta V_{F W H M}$ is the full-width at half-maximum of the line velocity.

The magnitude of the self-gravitational potential energy can be found from,

$$
\mathcal{G}=\frac{\eta G M^{2}}{R}
$$

where $\eta$ is a coefficient which is determined by the detailed density profile of the clump. For Bonner-Ebert spheres-which is assumed for the FIR cores- $-\eta=0.732$. 
Table 4.1: Energy Terms of the Virial Stability Evaluation

\begin{tabular}{c|cccccc}
\hline \hline & Thermal & Turbulence & Gravitational & Pressure & & \\
Source & $\begin{array}{c}2 \mathcal{U} \\
{[\mathrm{erg}]}\end{array}$ & $\begin{array}{c}2 \mathcal{T} \\
{[\mathrm{erg}]}\end{array}$ & $\begin{array}{c}\mathcal{G} \\
{[\mathrm{erg}]}\end{array}$ & $\begin{array}{c}\mathcal{X} \\
{[\mathrm{erg}]}\end{array}$ & $\begin{array}{c}2 \mathcal{U}+2 \mathcal{T} \\
{[\mathrm{erg}]}\end{array}$ & $\begin{array}{c}\mathcal{G}+\mathcal{X} \\
{[\mathrm{erg}]}\end{array}$ \\
\hline FIR 3 & $5.2 \times 10^{44}$ & $5.2 \times 10^{44}$ & $1.5 \times 10^{44}$ & $5.1 \times 10^{44}$ & $1.0 \times 10^{45}$ & $5.2 \times 10^{45}$ \\
FIR 4 & $4.0 \times 10^{44}$ & $4.7 \times 10^{44}$ & $1.3 \times 10^{44}$ & $3.8 \times 10^{44}$ & $0.9 \times 10^{45}$ & $3.9 \times 10^{45}$ \\
FIR 5 & $5.3 \times 10^{44}$ & $7.2 \times 10^{44}$ & $1.1 \times 10^{44}$ & $3.4 \times 10^{44}$ & $1.3 \times 10^{45}$ & $3.5 \times 10^{45}$ \\
FIR 6 & $7.0 \times 10^{44}$ & $1.5 \times 10^{45}$ & $2.6 \times 10^{44}$ & $3.2 \times 10^{44}$ & $2.2 \times 10^{45}$ & $8.6 \times 10^{45}$ \\
\hline
\end{tabular}

The external pressure term is approximated by,

$$
\mathcal{X}=2 \pi R^{3} P_{B}
$$

where $P_{B}$ is the pressure acting on the core boundary,

$$
P_{B}=n k T,
$$

where $n$ is the $\mathrm{H}_{2}$ number density which is approximated to be constant throughout the core radius. Table 4.1 shows the energy terms for each FIR core and the final sums of the left and right side of the inequality.

From the last two columns of Table 4.1, it is apparent that the sum of internal energy (column 6) is lower than the sum of external energy (column 7). From Eqn. 4.1, the evaluated FIR cores are all unstable against collapse. Although there were assumptions and approximations that contributed to the calculation of the virial theorem (turbulence calculations, the size and mass of the cores, etc.), the most reliable values for temperatures and densities from the $\mathrm{H}_{2} \mathrm{CO}$ transitions were used. The implication of contraction due to gravitational collapse of the cores supports the idea that FIRs 3-6 are advanced in their evolution. These results suggest that an observer studying NGC 2024 a million years from now will see a new generation of low mass stars where the molecular ridge is today. 


\section{Conclusion}

Observations from the JCMT were used to investigate the ridge of NGC 2024. The following points summarize the key findings of this thesis.

1. The two $H_{2} C O$ transition ratios, $3_{03} \rightarrow 2_{02} / 3_{22} \rightarrow 2_{21}$ and $5_{05} \rightarrow 4_{04} / 5_{23} \rightarrow 4_{22}$ are used to find gas kinetic temperatures for the ridge of NGC 2024. Fixed declination cuts across FIR core positions are used to investigate the temperature variation of the cores and their surroundings. Temperatures at core positions are found to be warm, between 45 and $85 \mathrm{~K}$. There is no observed variation in temperature between the core centers and their envelopes.

2. The formaldehyde line ratio $3_{03} \rightarrow 2_{02} / 5_{05} \rightarrow 4_{04}$ yields a $H_{2}$ number density of $\sim 2 \times 10^{6} \mathrm{~cm}^{-3}$ at FIR core positions. $\mathrm{H}_{2} \mathrm{CO}$ column densities are found in the range of $6 \times 10^{14}$ to $1 \times 10^{15} \mathrm{~cm}^{-2}$. $\mathrm{HCO}^{+}$column densities are found to range from $8 \times 10^{13} \mathrm{~cm}^{-2}$ to $3 \times 10^{14} \mathrm{~cm}^{-2}$. The variation in $\mathrm{H}_{2} \mathrm{CO}$ column density along cuts across FIR core positions is investigated, and reveals that for almost all cuts, the central core position displays the highest value.

3. Fractional abundances of $\mathrm{H}_{2} \mathrm{CO}$ and $\mathrm{HCO}^{+}$are obtained for the FIR core positions by taking the ratio of $\mathrm{H}_{2} \mathrm{CO}$ and $\mathrm{HCO}^{+}$column densities over $\mathrm{H}_{2}$ column densities derived from $850 \mu \mathrm{m}$ dust data (Johnstone et al., 2006). $\mathrm{H}_{2} \mathrm{CO}$ fractional abundances are found to be between $2 \times 10^{-9}$ and $5 \times 10^{-9}$, which agrees well with previously obtained fractional abundances for similar regions. $\mathrm{HCO}^{+}$column densities gave $\mathrm{HCO}^{+}$fractional abundances of $3 \times 10^{-10}-7 \times 10^{-10}$, which is low by an order of magnitude compared to values for other molecular clouds. The assumptions which were made to carry through $\mathrm{HCO}^{+}$calculations may be responsible for the discrepancy for the $\mathrm{HCO}^{+}$fractional abundances.

4. Core masses are derived by using SCUBA $850 \mu \mathrm{m}$ dust observations [from Johnstone et al. (2006)] and incorporating the temperatures found from formaldehyde transition ratios. The resulting values all give $\sim 2 \mathrm{M}_{\odot}$ within a radius of $15^{\prime \prime}$ for FIRs $3,4,5$, and 6 . These modest core masses make it unlikely that massive star formation will occur within the ridge. 
5. The derived core masses are used to evaluate the stability of the FIRs $3,4,5$, and 6 using the virial theorem. Energies are estimated by taking into account the previously derived physical properties (e.g. core sizes), and the properties obtained from formaldehyde transitions (e.g. temperature, density). The result suggests that the four FIR cores are unstable against collapse.

6. Visual extinctions towards mid-IR sources detected by Haisch et al. (2001) are used to obtain $H_{2}$ column densities at these source positions. $H_{2}$ column densities are obtained as well from the 850 $\mu \mathrm{m}$ map from Johnstone et al. (2006) at the same positions as the mid-IR sources. These two sets of column densities are compared to probe the orientation of the star cluster with respect to the dense dust and molecular gas. The majority of the detected mid-IR sources are located behind the molecular ridge, and a few sources suggest that they are embedded in a small envelope or circumstellar disk. 


\section{REFERENCES}

B. J. Anthony-Twarog. The H-beta distance scale for B stars - The Orion association. AJ, 87:1213-1222, August 1982.

J. A. Ball, D. Cesarsky, A. K. Dupree, L. Goldberg, and A. E. Lilley. Detection and Identification of Recombination Lines from an H i Region. ApJL, 162:L25+, October 1970.

R. Banerjee and R. E. Pudritz. Outflows and Jets from Collapsing Magnetized Cloud Cores. ApJ, 641: 949-960, April 2006.

P. J. Barnes and R. M. Crutcher. Orion B (NGC 2024). II - Hat Creek synthesis observations of the molecular core in the $\mathrm{J}=1$ to 0 line of $\mathrm{HCO}(+)$. ApJ, 351:176-188, March 1990.

P. J. Barnes, R. M. Crutcher, J. H. Bieging, J. W. V. Storey, and S. P. Willner. Orion B (NGC 2024). I VLA and IR observations of the H II region. ApJ, 342:883-907, July 1989.

M. R. Bate, I. A. Bonnell, and V. Bromm. The formation of a star cluster: predicting the properties of stars and brown dwarfs. MNRAS, 339:577-599, March 2003.

P. R. Bevington. Data reduction and error analysis for the physical sciences. New York: McGraw-Hill, $1969,1969$.

A. Bik, A. Lenorzer, L. Kaper, F. Comerón, L. B. F. M. Waters, A. de Koter, and M. M. Hanson. Identification of the ionizing source of NGC 2024. A\&A, 404:249-254, June 2003.

J. H. Black and S. P. Willner. Interstellar absorption lines in the infrared spectrum of NGC 2024 IRS 2. ApJ, 279:673-678, April 1984.

G. A. Blake, E. C. Sutton, C. R. Masson, and T. G. Phillips. Molecular abundances in OMC-1 - The chemical composition of interstellar molecular clouds and the influence of massive star formation. ApJ, 315:621-645, April 1987.

I. A. Bonnell and M. R. Bate. Accretion in stellar clusters and the collisional formation of massive stars. MNRAS, 336:659-669, October 2002. 
I. A. Bonnell, M. R. Bate, and S. G. Vine. The hierarchical formation of a stellar cluster. $M N R A S, 343$ : 413-418, August 2003.

T. L. Bourke and A. A. Goodman. Magnetic Fields in Molecular Clouds. In M. Burton, R. Jayawardhana, and T. Bourke, editors, Star Formation at High Angular Resolution, volume 221 of IAU Symposium, pages 83-+, September 2004.

D. A. Cesarsky. Detection of H137 $\alpha$ Recombination-Line Emission from an H i Region in the Direction of NGC 2024. ApJL, 167:L89+, August 1971.

C. J. Chandler and J. E. Carlstrom. Star Formation in the NGC 2024 Molecular Ridge. ApJ, 466:338-+, July 1996.

R. M. Crutcher, C. Henkel, T. L. Wilson, K. J. Johnston, and J. H. Bieging. VLA maps of formaldehyde absorption toward NGC 2024. ApJ, 307:302-312, August 1986.

J. Di Francesco, P. C. Myers, D. J. Wilner, N. Ohashi, and D. Mardones. Infall, Outflow, Rotation, and Turbulent Motions of Dense Gas within NGC 1333 IRAS 4. ApJ, 562:770-789, December 2001.

S. D. Doty, E. F. van Dishoeck, F. F. S. van der Tak, and A. M. S. Boonman. Chemistry as a probe of the structures and evolution of massive star-forming regions. $A \& A, 389: 446-463$, July 2002.

N. I. Evans. Studying Infall. In C. L. Curry and M. Fich, editors, SFChem 2002: Chemistry as a Diagnostic of Star Formation, proceedings of a conference held August 21-23, 2002 at University of Waterloo, Waterloo, Ontario, Canada N2L 3G1. Edited by Charles L. Curry and Michel Fich. NRC Press, Ottawa, Canada, 2003, p. 157., pages 157-+, 2003.

L. Fissel, D. Johnstone, Mitchell G. F., and Avery L. A dust continuum and molecular line study of NGC 2024. Submitted to ApJ, 2007.

A. Frey, D. Lemke, U. Fahrbach, and C. Thum. Near infrared observations of NGC 2024. A\&A, 74: 133-137, April 1979.

R. A. Gaume, K. J. Johnston, and T. L. Wilson. High spatial resolution 1.3 centimeter imaging of the NGC 2024 star-forming region. ApJ, 388:489-494, April 1992. 
R. Genzel and D. Downes. $\mathrm{H}_{2} \mathrm{O}$ in the Galaxy: sites of newly formed OB stars. $A \& A S, 30: 145-168$, October 1977 .

U. U. Graf, A. Eckart, R. Genzel, A. I. Harris, A. Poglitsch, A. P. G. Russell, and J. Stutzki. CO lines toward NGC 2024 and other star-forming regions - A closer look at the warm gas component. ApJ, 405: 249-267, March 1993.

G. L. Grasdalen. An Infrared Study of NGC 2024. ApJ, 193:373--, October 1974.

K. E. Haisch, Jr., E. A. Lada, and C. J. Lada. A Near-Infrared L-Band Survey of the Young Embedded Cluster NGC 2024. $A J, 120: 1396-1409$, September 2000.

K. E. Haisch, Jr., E. A. Lada, R. K. Piña, C. M. Telesco, and C. J. Lada. A Mid-Infrared Study of the Young Stellar Population in the NGC 2024 Cluster. AJ, 121:1512-1521, March 2001.

D. A. Harper. Far-Infrared Emission from H II Regions. 11. Multicolor Photometry of Selected Sources and 2/2 Resolution Maps of M42 and NGC 2024. ApJ, 192:557-576, September 1974.

F. P. Helmich. Dense Molecular Gas around Massive Young Stars. $\mathrm{PhD}$ thesis, , Leiden University, (1996), 1996.

P. T. P. Ho, Y.-L. Peng, J. M. Torrelles, J. F. Gomez, L. F. Rodriguez, and J. Canto. A flattened cloud core in NGC 2024. ApJ, 408:565-572, May 1993.

M. R. Hogerheijde, E. F. van Dishoeck, G. A. Blake, and H. J. van Langevelde. Tracing the Envelopes around Embedded Low-Mass Young Stellar Objects with HCO + and Millimeter-Continuum Observations. ApJ, 489:293-+, November 1997.

D. Johnstone, M. Fich, G. F. Mitchell, and G. Moriarty-Schieven. Large Area Mapping at 850 Microns. III. Analysis of the Clump Distribution in the Orion B Molecular Cloud. ApJ, 559:307-317, September 2001 .

D. Johnstone, H. Matthews, and G. F. Mitchell. Large Area Mapping at $850 \mu \mathrm{m}$. IV. Analysis of the Clump Distribution in the Orion B South Molecular Cloud. ApJ, 639:259-274, March 2006. 
G. R. Knapp and M. Morris. Mass loss from evolved stars. III - Mass loss rates for fifty stars from CO J = 1-0 observations. ApJ, 292:640-669, May 1985 .

E. A. Lada, J. Bally, and A. A. Stark. An unbiased survey for dense cores in the LYNDS 1630 molecular cloud. ApJ, 368:432-444, February 1991a.

E. A. Lada, N. J. Evans, II, D. L. Depoy, and I. Gatley. A 2.2 micron survey in the L1630 molecular cloud. ApJ, 371:171-182, April 1991b.

R. B. Larson. KEY ISSUES REVIEW: Insights from simulations of star formation. Reports of Progress in Physics, 70:337-356, March 2007.

D. C. Lis, J. E. Carlstrom, and T. G. Phillips. Protostellar condensations in the core of NGC 2024. ApJ, 370:583-589, April 1991.

M. N. Machida, S.-i. Inutsuka, and T. Matsumoto. Outflows Driven by Giant Protoplanets. ApJL, 649: L129-L132, October 2006.

J. G. Mangum and A. Wootten. Formaldehyde as a probe of physical conditions in dense molecular clouds. ApJS, 89:123-153, November 1993.

J. G. Mangum, A. Wootten, and M. Barsony. The Kinetic Temperature Structure within NGC 2024. ApJ, 526:845-853, December 1999.

R. Mauersberger, T. L. Wilson, P. G. Mezger, R. Gaume, and K. J. Johnston. The internal structure of molecular clouds. III - Evidence for molecular depletion in the NGC 2024 condensations. $A \& A, 256$ : 640-651, March 1992.

P. G. Mezger, R. Chini, E. Kreysa, J. E. Wink, and C. J. Salter. Dust emission at submillimeter wavelengths from cloud cores and protostellar condensations in NGC 2024 and S 255 IR. A\&GA, 191:44-56, February 1988.

P. G. Mezger, A. W. Sievers, C. G. T. Haslam, E. Kreysa, R. Lemke, R. Mauersberger, and T. L. Wilson. Dust emission from star forming regions. II - The NGC 2024 cloud core: Revisited. $A \& A, 256: 631-639$, March 1992. 
G. F. Mitchell, D. Johnstone, G. Moriarty-Schieven, M. Fich, and N. F. H. Tothill. A Submillimeter Dust and Gas Study of the Orion B Molecular Cloud. ApJ, 556:215-229, July 2001.

T. J. T. Moore and C. J. Chandler. Near-infrared observations of NGC 2024 - Identification of a 2-micron source within a dense core. MNRAS, 241:19P-24P, November 1989.

T. J. T. Moore, C. J. Chandler, W. K. Gear, and C. M. Mountain. CS(J = 7-6) from heated gas near the embedded sources in NGC 2024. MNRAS, 237:1P-6P, March 1989.

T. J. T. Moore and T. Yamashita. A Near-Infrared Reflection Nebula Associated with NGC 2024 FIR 4. $A p J, 440: 722-+$, February 1995.

J. S. Richer. A compact CO bipolar outflow from NGC 2024 FIR6. MNRAS, 245:24P-27P, July 1990.

J. S. Richer, R. E. Hills, and R. Padman. A fast CO jet in Orion B. MNRAS, 254:525-538, February 1992.

J. S. Richer, R. E. Hills, R. Padman, and A. P. G. Russell. High-resolution molecular line observations of the core and outflow in Orion B. MNRAS, 241:231-246, November 1989.

L. F. Rodríguez, Y. Gómez, and B. Reipurth. A Cluster of Compact Radio Sources in NGC 2024 (Orion B). $A p J, 598: 1100-1106$, December 2003.

K. Schreyer, T. Henning, F. F. S. van der Tak, A. M. S. Boonman, and E. F. van Dishoeck. The young intermediate-mass stellar object AFGL 490 - A disk surrounded by a cold envelope. A $A A$ A, 394:561-583, November 2002.

A. Schulz, R. Guesten, R. Zylka, and E. Serabyn. Dense clumps in NGC 2024 - Protostellar condensations? $A \mathscr{E A}, 246: 570-586$, June 1991.

N. Z. Scoville, A. I. Sargent, D. B. Sanders, M. J. Claussen, C. R. Masson, K. Y. Lo, and T. G. Phillips. High-resolution mapping of molecular outflows in NGC 2071, W49, and NGC 7538. ApJ, 303:416-432, April 1986.

F. H. Shu. Self-similar collapse of isothermal spheres and star formation. ApJ, 214:488-497, June 1977. 
F. H. Shu, F. C. Adams, and S. Lizano. Star formation in molecular clouds - Observation and theory. ARA\&A, 25:23-81, 1987.

B. T. Soifer and H. S. Hudson. Submillimeter Observations of the Orion Nebula and NGC 2024. ApJL, 191:L83+, July 1974.

R. I. Thompson, H. A. Thronson, Jr., and B. G. Campbell. The nature of NGC 2024 - Near-infrared spectroscopy of IRS 1 and IRS 2. ApJ, 249:622-627, October 1981.

H. A. Thronson, Jr., P. R. Schwartz, H. A. Smith, W. Glaccum, D. A. Harper, R. F. Loewenstein, J. Smith, and C. J. Lada. NGC 2024 - Far-infrared and radio molecular observations. ApJ, 280:154-162, May 1984.

F. F. S. van der Tak, E. F. van Dishoeck, and P. Caselli. Abundance profiles of $\mathrm{CH}_{3} \mathrm{OH}$ and $\mathrm{H}_{2} \mathrm{CO}$ toward massive young stars as tests of gas-grain chemical models. $A \& A, 361: 327-339$, September 2000.

E. F. van Dishoeck, D. J. Jansen, and T. G. Phillips. Submillimeter observations of the shocked molecular gas associated with the supernova remnant IC 443. $A \& A, 279: 541-566$, November 1993.

A. E. Visser, J. S. Richer, and C. J. Chandler. A SCUBA survey of compact dark Lynds clouds. $M N R A S$, 323:257-269, May 2001.

A. E. Visser, J. S. Richer, and C. J. Chandler. Completion of a SCUBA Survey of Lynds Dark Clouds and Implications for Low-mass Star Formation. $A J$, 124:2756-2789, November 2002.

A. E. Visser, J. S. Richer, C. J. Chandler, and R. Padman. Submillimetre continuum images of the NGC 2024 star-forming ridge. MNRAS, 301:585-592, December 1998.

D. Ward-Thompson, D. Nutter, S. Bontemps, A. Whitworth, and R. Attwood. SCUBA observations of the Horsehead nebula - what did the horse swallow? MNRAS, 369:1201-1210, July 2006.

H. Wiesemeyer, R. Guesten, J. E. Wink, and H. W. Yorke. High resolution studies of protostellar condensations in NGC 2024. $A \& A, 320: 287-299$, April 1997.

T. L. Wilson, D. M. Mehringer, and H. R. Dickel. High resolution observations of $\mathrm{C}^{\wedge} 18^{\wedge} \mathrm{O}$ and the 2.7 MM continuum toward NGC 2024. AEA, 303:840-+, November 1995. 
C. H. Young and N. J. Evans, II. Evolutionary Signatures in the Formation of Low-Mass Protostars. ApJ, 627:293-309, July 2005.

This research has made use of the NASA/ IPAC Infrared Science Archive, which is operated by the Jet Propulsion Laboratory, California Institute of Technology, under contract with the National Aeronautics and Space Administration. 